University of Tennessee Health Science Center

UTHSC Digital Commons

\title{
HP Turns 17: T helper 17 cell response during hypersensitivity pneumonitis (HP) and factors controlling it
}

\author{
Hossam Abdelsamed \\ University of Tennessee Health Science Center
}

Follow this and additional works at: https://dc.uthsc.edu/dissertations

Part of the Medical Immunology Commons, Medical Microbiology Commons, and the Medical Molecular Biology Commons

\section{Recommended Citation}

Abdelsamed, Hossam, "HP Turns 17: T helper 17 cell response during hypersensitivity pneumonitis (HP) and factors controlling it" (2012). Theses and Dissertations (ETD). Paper 6. http://dx.doi.org/10.21007/ etd.cghs.2012.0002.

This Dissertation is brought to you for free and open access by the College of Graduate Health Sciences at UTHSC Digital Commons. It has been accepted for inclusion in Theses and Dissertations (ETD) by an authorized administrator of UTHSC Digital Commons. For more information, please contact jwelch30@uthsc.edu. 


\title{
HP Turns 17: T helper 17 cell response during hypersensitivity pneumonitis (HP) and factors controlling it
}

\author{
Abstract \\ Hypersensitivity Pneumonitis (HP) is an interstitial lung disease caused by repeated inhalation of a wide \\ range of environmental antigens. It is characterized by alveolitis, granuloma formation, and fibrosis. Since \\ $\mathrm{HP}$ is a T cell-mediated disease, it is important to determine the type of T cell response associated with \\ granuloma formation and the factors that control this response. We hypothesized that HP is associated \\ with a predominant Th17 cell response where both T-bet and TLRs 2 and 9 are controlling T cell response \\ during HP. The results demonstrated a predominant Th17 response associated with granuloma formation \\ in the lungs of C57BL/6J mice during granulomatous and chronic HP. We also found that T-bet KO mice \\ exposed to Saccharopolyspora rectivirgula (SR) were characterized by exacerbated Th17 cell response \\ accompanied by an increase in granuloma formation and collagen production in the lungs compared to \\ WT exposed mice. In an attempt to find other factors regulating Th17 development, previous studies in \\ our lab showed that TLR2/9 double knockout (DKO) exposed mice were characterized by a decrease in \\ the percentage of Th17 cells in their lungs compared to WT mice. Consequently, we hypothesized that the \\ decrease in Th17 response is attributed to defect in phagocytosis, or cytokines production, or antigen \\ presentation ability of TLR2/9 DKO antigen presenting cells (APCs). In vitro, we found no significant \\ difference in the phagocytosis ability of both WT and TLR2/9 DKO BMDMs (Bone Marrow Derived \\ Macrophages) or BMDCs (Bone Marrow Derived Dendritic Cells). In vivo, there was no significant \\ difference in the phagocytosis ability of both WT and TLR2/9 DKO alveolar macrophages. Although we \\ found that both TLRs 2 and 9 were regulating cytokine production e.g. IL-6, IL-10, and TNF $\alpha$ from BMDMs, \\ we did not find a defect in antigen presentation ability of both WT and TLR2/9 DKO splenic APCs. In \\ conclusion, these findings suggested the following: (1) Both granulomatous and chronic HP is associated \\ with a predominant Th17 response and granuloma formation; (2) T-bet plays a role in controlling disease \\ severity and Th17 development during HP; (3) TLR2 and 9 do not affect APCs' ability to phagocytose SR \\ but they affect cytokine production; and (4) Both WT and TLR2/9 DKO APCs are equally efficient in \\ presenting SR antigen to T cells.

\section{Document Type} \\ Dissertation \\ Degree Name \\ Doctor of Philosophy (Medical Science)

\section{Program} \\ Microbiology, Molecular Biology and Biochemistry \\ Research Advisor \\ Elizabeth A. Fitzpatrick, Ph.D. \\ Keywords \\ Th17, T-bet, Hypersensitivity pneumonitis

\section{Subject Categories} \\ Medical Immunology | Medical Microbiology | Medical Molecular Biology | Medicine and Health Sciences
}




\title{
HP TURNS 17: T HELPER 17 CELL RESPONSE DURING HYPERSENSITIVITY PNEUMONITIS (HP) AND FACTORS CONTROLLING IT
}

\author{
A Dissertation \\ Presented for \\ The Graduate Studies Council \\ The University of Tennessee \\ Health Science Center
}

\begin{abstract}
In Partial Fulfillment
Of the Requirements for the Degree

Doctor of Philosophy

From The University of Tennessee
\end{abstract}

By

Hossam Abdelsamed

May 2012 
Portions of Chapter 3 (c) 2012 by BioMed Central Ltd.

All other materials (C) 2012 by Hossam Abdelsamed. All rights reserved. 


\section{DEDICATION}

I dedicate this work to my parents,

Aly Abdelsamed and Salwa Fahmy, to my wife,

Amira Ahmed and to my future students. 


\begin{abstract}
Hypersensitivity Pneumonitis (HP) is an interstitial lung disease caused by repeated inhalation of a wide range of environmental antigens. It is characterized by alveolitis, granuloma formation, and fibrosis. Since HP is a T cell-mediated disease, it is important to determine the type of $\mathrm{T}$ cell response associated with granuloma formation and the factors that control this response. We hypothesized that HP is associated with a predominant Th17 cell response where both T-bet and TLRs 2 and 9 are controlling T cell response during HP. The results demonstrated a predominant Th17 response associated with granuloma formation in the lungs of C57BL/6 J mice during granulomatous and chronic HP. We also found that T-bet KO mice exposed to Saccharopolyspora rectivirgula (SR) were characterized by exacerbated Th17 cell response accompanied by an increase in granuloma formation and collagen production in the lungs compared to WT exposed mice. In an attempt to find other factors regulating Th17 development, previous studies in our lab showed that TLR2/9 double knockout (DKO) exposed mice were characterized by a decrease in the percentage of Th17 cells in their lungs compared to WT mice. Consequently, we hypothesized that the decrease in Th17 response is attributed to defect in phagocytosis, or cytokines production, or antigen presentation ability of TLR2/9 DKO antigen presenting cells (APCs). In vitro, we found no significant difference in the phagocytosis ability of both WT and TLR2/9 DKO BMDMs (Bone Marrow Derived Macrophages) or BMDCs (Bone Marrow Derived Dendritic Cells). In vivo, there was no significant difference in the phagocytosis ability of both WT and TLR2/9 DKO alveolar macrophages. Although we found that both TLRs 2 and 9 were regulating cytokine production e.g. IL-6, IL-10, and TNF $\alpha$ from BMDMs, we did not find a defect in antigen presentation ability of both WT and TLR2/9 DKO splenic APCs. In conclusion, these findings suggested the following: (1) Both granulomatous and chronic HP is associated with a predominant Th17 response and granuloma formation; (2) T-bet plays a role in controlling disease severity and Th17 development during HP; (3) TLR2 and 9 do not affect APCs' ability to phagocytose SR but they affect cytokine production; and (4) Both WT and TLR2/9 DKO APCs are equally efficient in presenting SR antigen to T cells.
\end{abstract}




\section{TABLE OF CONTENTS}

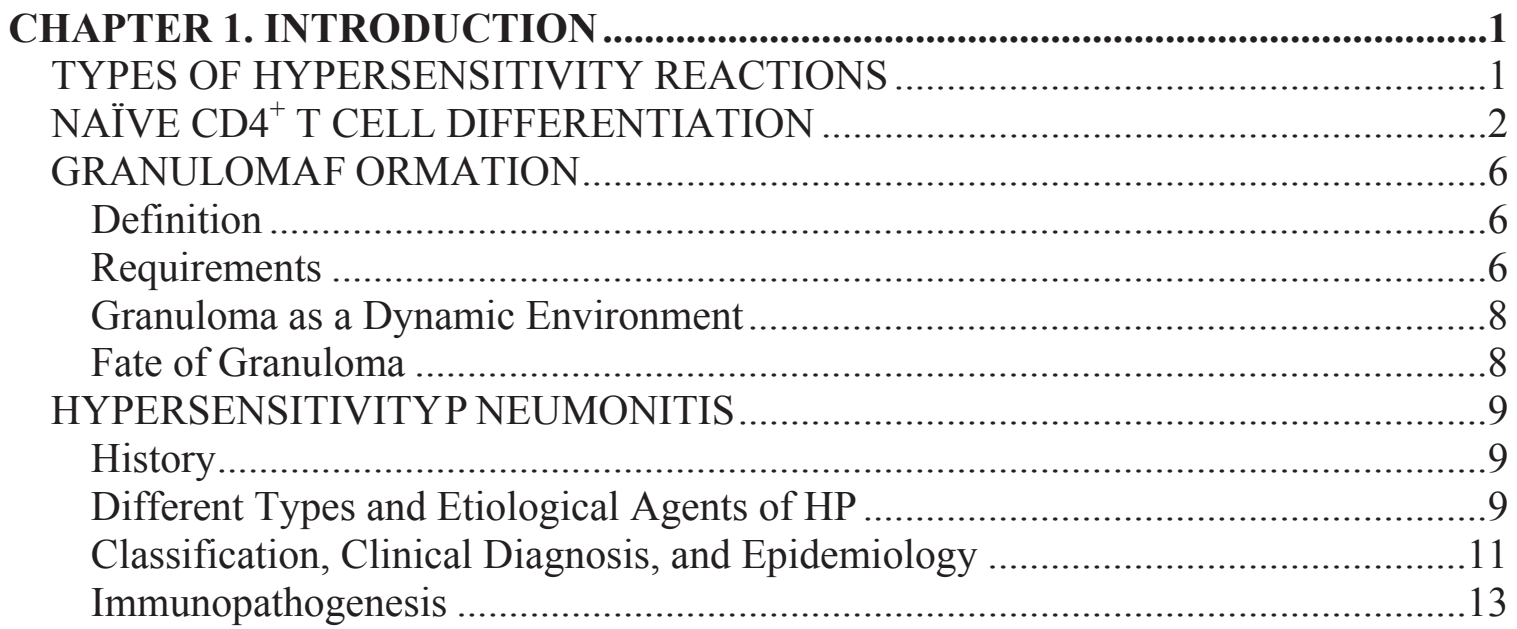

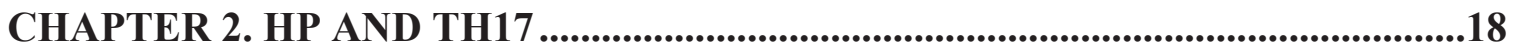

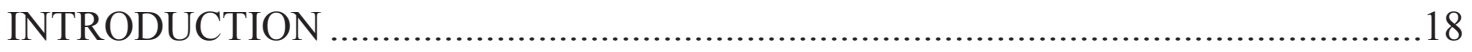

Th17 Cell as a Distinct CD4 $4^{+}$T Helper Cell.......................................................18

Effector Function of Th17 Cytokines ……………….......................................19

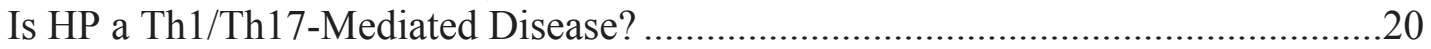

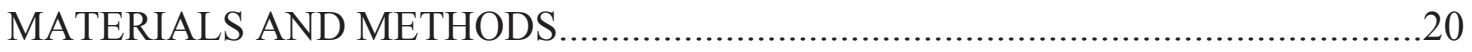

Bacterial Preparation and Exposure Protocol ………….......................................2

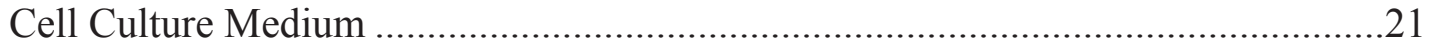

Bronchoalveolar Lavage (BAL) and Lung Cell Isolation........................................21

RNA Extraction and Semi-Quantitative RT-PCR ..............................................22

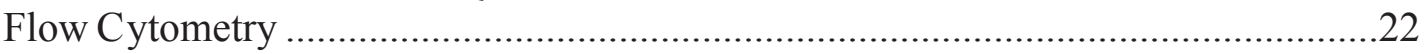

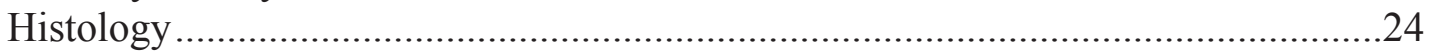

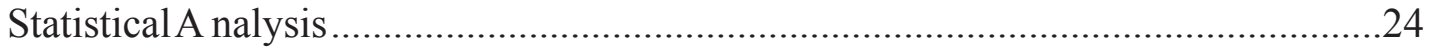

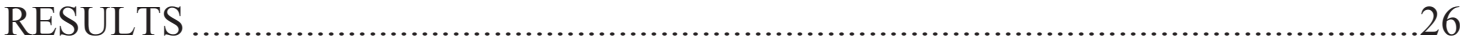

Characterization of BAL Composition during Acute HP .........................................26

Expression of IL-17 and CXCL2 during Acute Phase of HP ...................................26

Acute HP Is Associated with Th17 Differentiation and Effector Cytokines ..............27

Characterization of BAL Composition during Granulomatous HP ...........................27

Granuloma Formation during Granulamatous HP ...................................................30

Granulomatous HP Is Associated with Activated CD4 $4^{+}$T Cells .................................30

Granulomatous HP Is Associated with Th17 Immune Response .................................31

Characterization of BALF during Chronic HP .......................................................35

Chronic HP Is Associated with Granuloma Formation ..............................................35

Chronic HP Is Associated with Activated $\mathrm{CD}^{+}$and $\mathrm{CD} 8^{+} \mathrm{T}$ Cells ...........................35

Chronic HP Is Associated with a Th17 Immune Response ……………….................38

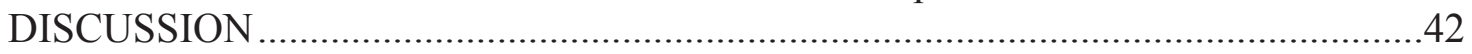

Acute HP: Neutrophilic Alveolitis and IL-17 Expression ........................................42

Granulomatous HP and the Th17 Response ………….........................................42

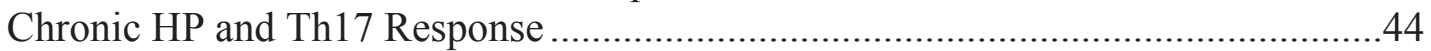


Th17 Response and HP: A Correlation Not a Cause and Effect Study .....

CHAPTER 3. T-BET IN HP .............................................................................47

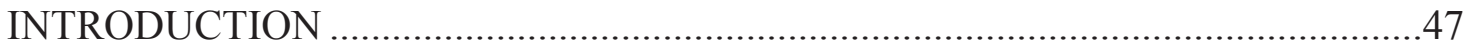

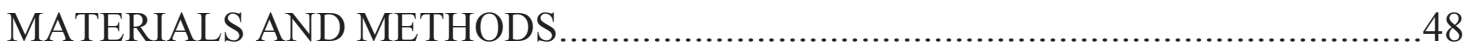

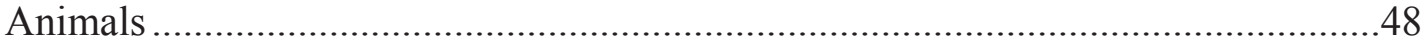

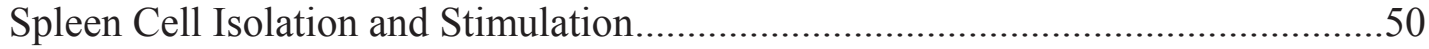

Neutrophil Cell Sorting and Stimulation ............................................................50

RNA Extraction and Real-Time PCR ................................................................50

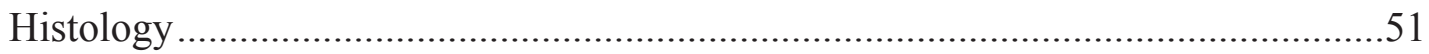

Measurement of Lung Collagen Content .............................................................51

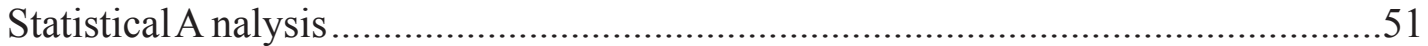

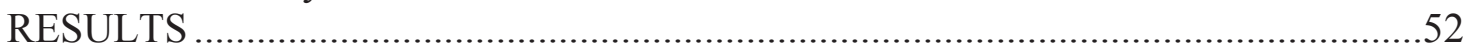

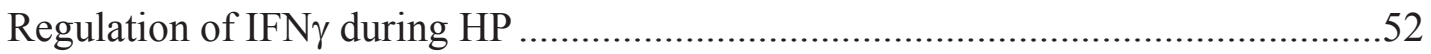

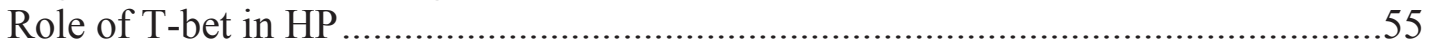

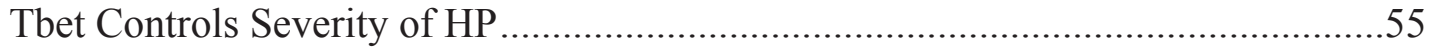

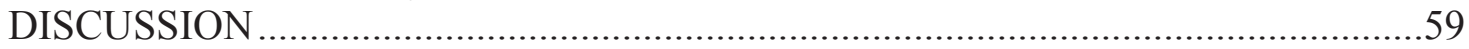

\section{CHAPTER 4. EFFECT OF TLR2 AND 9 IN APCS ON TH17 CELL}

POLARIZATION AND EFFECTOR FUNCTION ..................................................63

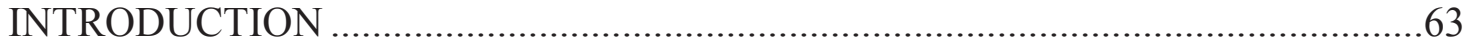

Antigen Processing, Presentation, and Recognition Processes...............................63

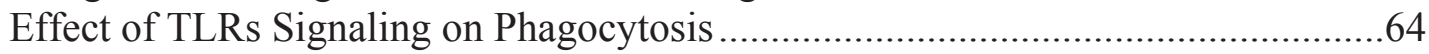

Effect of TLRs Signaling on Costimulatory Molecules and Cytokine Expression ...65

The Role of TLRs in Innate and Adaptive Immunity during HP ...........................66

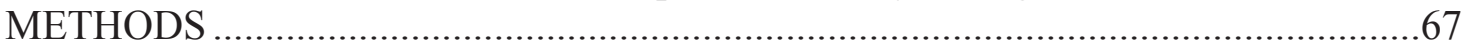

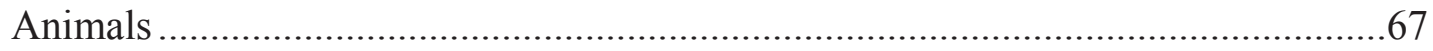

Antigen Presentation Assay ................................................................................67

Generation of Bone Marrow Derived Macrophages and Dendritic .........................68

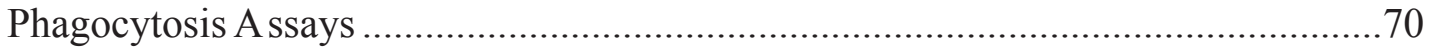

In vitro Stimulation of BMDMs Using SR .......................................................... 71

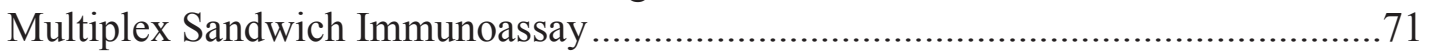

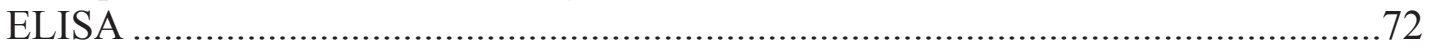

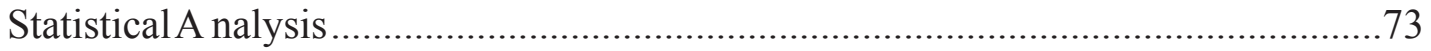

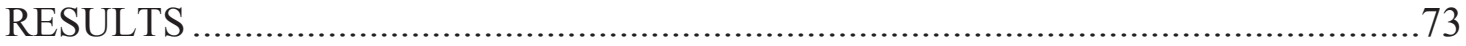

TLRs 2 and 9 Did Not Affect Phagocytosis Ability of BMDCs ..............................73

TLRs 2 and 9 Did Not Affect Phagocytosis Ability of BMDMs...............................73

TLRs 2 and 9 Did Not Affect Phagocytosis Ability of AMs..................................75

TLRs 2 and 9 Did Not Affect the Antigen Presentation Ability of Splenic APCs ....83

Effect of TLRs 2 and 9 on Cytokine Expression from BMDMs ...........................87

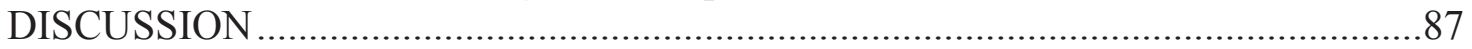

TLRs 2 and 9 Did Not Affect the Phagocytosis Ability of DMDMs and BMDCs ...87

TLRs 2 and 9 Did Not Affect the Antigen Presentation Ability of Splenic APCs ....90

TLRs 2 and 9 and MyD88 Affect Cytokine Expression by BMDMs 
CHAPTER 5. DISCUSSION ............................................................................................93

HP IS ASSOCIATED WITH A PREDOMINANT TH17 RESPONSE .......................93

CORRELATION BETWEEN T-BET, DISEASE SEVERITY AND TH17

RESPONSE DURING HP

EFFECT OF TLRS 2 AND 9 ON TH17 RESPONSE DURING HP

CHAPTER 6. CONCLUDING REMARKS....................................................................95

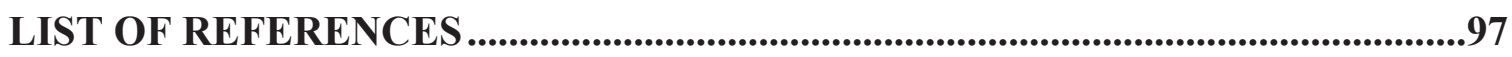

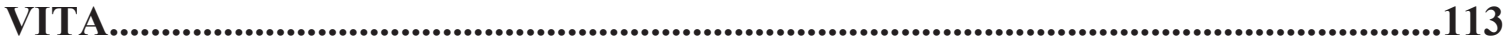




\section{LIST OF TABLES}

Table 1-1. Different forms of HP showing putative antigens and their source..............10

Table 2-1. $\quad$ List of PCR primers used for PCR ........................................................23

Table 2-2. Characterization of innate and adaptive immune cell surface and

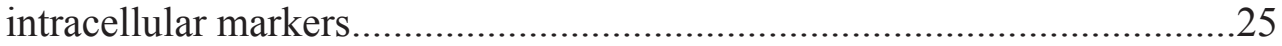

Table 2-3. C57BL/6J mice develop alveolitis and airway neutrophilia following acute exposure to SR compared to unexposed mice ..................................26

Table 2-4. C57BL/6J mice develop lymphocytic alveolitis during granulomatous

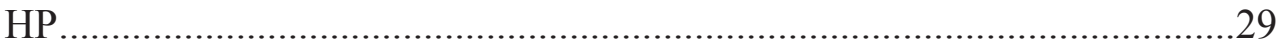

Table 2-5. Characterization of BAL cells during chronic HP ....................................36 


\section{LIST OF FIGURES}

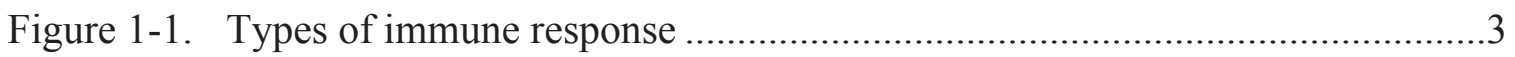

Figure 1-2. Differentiation of naïve $\mathrm{T}$ cell to different subsets of effector $\mathrm{CD}^{+} \mathrm{T}$ cells.

Figure 2-1. Three week SR exposure …...........................................................21

Figure 2-2. Expression of IL-17 and CXCL2 (MIP-2) during acute HP ......................28

Figure 2-3. mRNA expression of Th17 cytokines during acute HP ...........................28

Figure 2-4. Peribronchiolar granuloma formation following 3 weeks of SR exposure...30

Figure 2-5. Cell surface staining showing the average percentage of different cell populations in the lung interstium of unexposed and SR exposed mice following 3 weeks of SR exposure

Figure 2-6. mRNA expression of different cytokines and T cell transcription factors from unexposed and SR exposed mice during granulomatous HP

Figure 2-7. Intracellular cytokine staining showing percentage of Th1 and Th17 cells in the lungs following 3 weeks of SR exposed mice

Figure 2-8. Peribronchiolar granuloma formation following 25 weeks of SR exposure

Figure 2-9. Cell surface staining showing the average percentage of different cell populations in the lung interstium of unexposed and SR exposed mice following 25 weeks of SR exposure

Figure 2-10. mRNA expression of different cytokines and $\mathrm{T}$ cell transcription factors from unexposed and SR exposed mice during chronic HP

Figure 2-11. Intracellular cytokine staining showing percentage of Th17 and Th1 cells in the lungs following 25 weeks of SR exposure

Figure 3-1. Role of T-bet in differentiation of different subsets $\mathrm{CD}^{+}{ }^{+}$helper T cells ....49

Figure 3-2. SR induces IFN $\gamma$ from spleen cells indirectly through cytokines..... 53

Figure 3-3. Neither IL-12 nor IL-23 alone stimulates IFN $\gamma$ production by neutrophils..54

Figure 3-4. IFN $\gamma$ production from spleen is T-bet dependent following SR stimulation. 
Figure 3-5. Real-time PCR showing expression levels of IL-17 and IFN $\gamma$ in lungs of WT and T-bet KO mice following 3 weeks of SR exposure

Figure 3-6. Granuloma formation in WT and T-bet KO mice ......................................58

Figure 3-7. Increased collagen content in the lungs of T-bet KO mice during HP ........59

Figure 4-1. Steps for setting up antigen presentation assay.............................................68

Figure 4-2. Detection of internalized CFSE labeled SR in BMDCs using flow cytometry...... .74

Figure 4-3. Detection of internalized CFSE labeled SR in BMDMs using flow cytometry. . .76

Figure 4-4. Detection of internalized TAMRA labeled SR in BMDMs using confocal microscopy

Figure 4-5. Detection of internalized CFSE labeled SR in AMs using flow cytometry. .82

Figure 4-6. Detection of internalized TAMRA labeled SR in AMs using confocal microscopy

Figure 4-7. TLRs 2 and 9 did not affect antigen presentation ability of splenic APCs...86

Figure 4-8. Expression of IL-6, TNF $\alpha$, IL-10, and IL-12p70 from WT, TLRs 2/9 DKO and MyD88 KO BMDMs following in vitro SRs timulation .88

Figure 6-1. Overall HP model .96 


\section{LIST OF ABBREVIATIONS}

\begin{tabular}{|c|c|}
\hline AhR & Aryl Hydrocarbon Receptor \\
\hline $\mathrm{AhR}^{d / d}$ & Aryl Hydrocarbon Receptor Mutant Mice \\
\hline AMs & Alveolar Macrophages \\
\hline APCs & Antigen Presenting Cells \\
\hline BAL & Bronchoalveolar Lavage \\
\hline BALF & Bronchoalveolar Lavage Fluid \\
\hline BMDCs & Bone Marrow-Derived Dendritic Cells \\
\hline BMDMs & Bone Marrow-Derived Macrophages \\
\hline CLIP & Class II-Associated Ii-Derived Peptide \\
\hline DC & Dendritic Cells \\
\hline DKO & Double Knockout \\
\hline EAE & Experimental Autoimmune Encephalomyelitis \\
\hline EAU & Experimental Autoimmune Uveitis \\
\hline ECM & Extracellular Matrix (Proteins) \\
\hline ELISA & Enzyme-Linked Immunosorbent Assay \\
\hline EMT & Epithelial-Mesenchymal Transition \\
\hline ER & Endoplasmic Reticulum \\
\hline FLD & Farmer's Lung Disease \\
\hline GKO & IFN $\gamma$ Knockout \\
\hline GM-CSF & Granulocyte/Macrophage Colony Stimulating Factor \\
\hline $\mathrm{H} \& \mathrm{E}$ & Hematoxylin and Eosin (Stain) \\
\hline HKSR & Heat Killed SR \\
\hline HP & Hypersensitivity Pneumonitis \\
\hline HRCT & High Resolution Computed Tomography \\
\hline IACUC & Institutional Animal Care and Use Committee \\
\hline IFN $\gamma$ & Interferon Gamma \\
\hline $\mathrm{Ii}$ & Invariant Chain \\
\hline IL & Interleukin \\
\hline iTregs & Induced Tregs \\
\hline LCMV & Lymphocytic Choriomeningitis Virus \\
\hline MFI & Mean Fluorescence Intensity \\
\hline MHCI & Major Histocompatabilty Complex Molecule Class I \\
\hline MHCII & Major Histocompatabilty Complex Molecule Class II \\
\hline MS & Multiple Sclerosis \\
\hline PAL & Peptidoglycan-Associated Lipoprotein \\
\hline PAMPs & Pathogen Associated Molecular Patterns \\
\hline PRR & Pathogen Recognition Receptor \\
\hline RBCs & Red Blood Cells \\
\hline ROR & Retinoid-Related Orphan Receptor \\
\hline SD & Standard Deviation \\
\hline SR & Saccharopolyspora Rectivirgula \\
\hline TCR & T Cell Receptor \\
\hline TIR & Toll/IL-1 Receptor \\
\hline
\end{tabular}


TLRs

TNF $\alpha$
Toll-Like Receptors

Tumor Necrosis Factor Alpha 


\section{CHAPTER 1. INTRODUCTION}

Hypersensitivity Pneumonitis (HP) is an interstitial lung disease caused by repeated inhalation of a wide range of environmental antigens. Alveolitis, granuloma formation, and fibrosis are the hallmarks of the disease. Fibrosis is irreversible and associated with significant morbidity and mortality. To date, the only available treatments are corticosteroids and avoiding environmental antigen, and as such it is crucial to understand the immunopathogenic mechanisms underlying the disease, which may lead to development of novel therapies. Since several studies showed that HP is a $\mathrm{T}$ cell-mediated disease, the main goals of our studies were as follows:

1- Investigate the T cell response (Th1 or Th17) during HP

2- Investigate the effect of T-bet (T helper 1 cell transcription factor) on $\mathrm{T}$ cell response and disease severity during HP

3- Determine whether TLRs 2 and 9 in antigen presenting cells contribute to the Th1 or Th17 response during HP

\section{TYPES OF HYPERSENSITIVITY REACTIONS}

During our daily life, we are exposed to a wide range of antigens from different sources. The main function of our immune system is to deal with them in an appropriate way without damaging the host. For example, infectious agents such as bacteria, viruses, fungi, and parasites should be cleared efficiently by our immune system; while innocuous agents such as pollen grains and foods should not induce an immune response. However, in some circumstances a host will develop an exaggerated immune response against an inciting agent and it is the immune response that causes pathology. Therefore, the patient is hypersensitive against an inciting agent by developing hypersensitivity reaction. Hypersensitivity reactions are mediated by both arms of adaptive immunity, i.e. humoral immunity (antibody mediated) and cellular immunity (cell mediated). According to Gell and Coombs, hypersensitivity reactions are classified into four types: Type I (immediate hypersensitivity), II (cytotoxic hypersensitivity), III (immune complex mediated hypersensitivity), and IV (delayed or cell-mediated hypersensitivity) [1]. Type I Hypersensitivity is mediated by IgE antibodies, where hay fever or allergic asthma are considered as the classic examples of this type of hypersensitivty. Type I Hypersensitivy is initiated by activation of Th2 cells by allergens resulting in IgE production. $\operatorname{IgE}$ antibodies bind to mast cells, sensitizing them to subsequent exposures. With subsequent exposures, allergens bind to IgE on surface of mast cells causing degranulation of mast cells and the release of histamines causing contraction of smooth muscles, vasodilatation, and, increase in vascular permeability [2].

Type II hypersensitivity is mediated by $\operatorname{IgG}$ and $\operatorname{IgM}$ antibodies directed towards target antigens on the cell surface. These antibodies bind to their antigens on cells and are recognized by macrophages via their $\mathrm{Fc}$ receptors resulting in killing or cytolysis through complement activation. Hemolytic anemia (destruction of RBCs) and thrombocytopenia 
(destruction of platelets) are the most common examples of type II hypersensitivity. Type III hypersensitivity develops in sensitized individuals who have IgG directed against a specific antigen. Upon subsequent exposure to the antigen the individual forms antibodyantigen immune complexes which get deposited in the tissues. Normally, large immune complexes are cleared by phagocytes; however small immune complexes, such as those that occur in type III are not phagocytosed and tend to deposit in blood vessels causing tissue damage [2]. Type IV hypersensitivity is not mediated by antibodies as the previous types; instead it is cell-mediated. The specific antigen is presented by antigen presenting cells (APCs) to effector T cells, thereby stimulating cytokine release. The example of this type is granulomatous inflammation which will be discussed in detail later on in this chapter. The Tuberculin test is the most common example that exemplifies the sequence of cellular events in Type IV Hypersensitivity. Intradermal injection of tuberculin (carbohydrates and peptides from M. tuberculosis) elicits a T cell-mediated inflammatory response caused by Th1 cells. Th1 cells secrete IFN $\gamma$ and TNF $\beta$ once they recognize the antigen presented by APCs. These cytokines enhance expression of adhesion molecules on the endothelium and increase vascular leakiness resulting in recruitment of plasma cells and accessory cells [1]. Hypersensitivity Pneumonitis (HP) is classified under both type III and type IV hypersensitivity $[3,4]$ for several reasons that will be discussed in detail later in this chapter.

\section{NAÏVE CD4 ${ }^{+}$T CELL DIFFERENTIATION}

There are two types of immune response: innate (early) and adaptive (late) immune response. Adaptive immunity is further classified into cell-mediated (T cells) and humoral (antibodies secreted by $\mathrm{B}$ cells). $\mathrm{T}$ cells are divided into $\mathrm{CD} 4{ }^{+} \mathrm{T}$ cells (Helper T cells) and $\mathrm{CD} 8^{+} \mathrm{T}$ (Cytotoxic $\mathrm{T}$ cells) (Fig. 1-1). The term helper stems from these T cells' ability to help B cells for antibody class switching and activation of macrophages. $\mathrm{CD} 8^{+} \mathrm{T}$ cells are called cytotoxic $\mathrm{T}$ cells since they directly kill host cells infected with intracellular microorganisms by secretion of perforins and granzymes.

Once $\mathrm{T}$ cells are generated in the thymus (primary lymphoid organ), they exit to the circulation as naïve $\mathrm{T}$ cells. Naïve $\mathrm{CD} 4^{+} \mathrm{T}$ cells have not yet encountered a foreign antigen in their life cycle and they continuously circulate between blood and lymph nodes (secondary lymphoid organs). If they encounter a foreign antigen in the lymph node, they will become activated and differentiate into an effector $\mathrm{CD}_{4}^{+} \mathrm{T}$ cell. A naïve $\mathrm{T}$ cell only encounters an antigen if it is presented by dendritic cells (DCs) in the lymph node. DCs function to capture foreign antigens at the site of infection, process the antigen forming a complex between the peptide and Major histocompatibility complex molecule class II (MHCII) molecules then migrate to the draining lymph nodes to present the antigen/MHC II complex to naïve CD4 ${ }^{+} \mathrm{T}$ cells [5]. During presentation, DCs provide three signals to naïve $\mathrm{T}$ cells; signal 1 is processed antigen /MHC II complex engaging antigen specific $\mathrm{T}$ cell receptor (TCR). Signal 2 is engagement of costimulatory molecules; e.g. CD80 and CD86 by CD28 on the T cell [6] resulting in upregulation of IL-2 production and hence T cell proliferation [7]. Signal 3 is cytokine stimulation that directs naïve $\mathrm{CD}^{+} \mathrm{T}$ cells to differentiate into effector Th1, Th2, Th17, or iTregs cells 


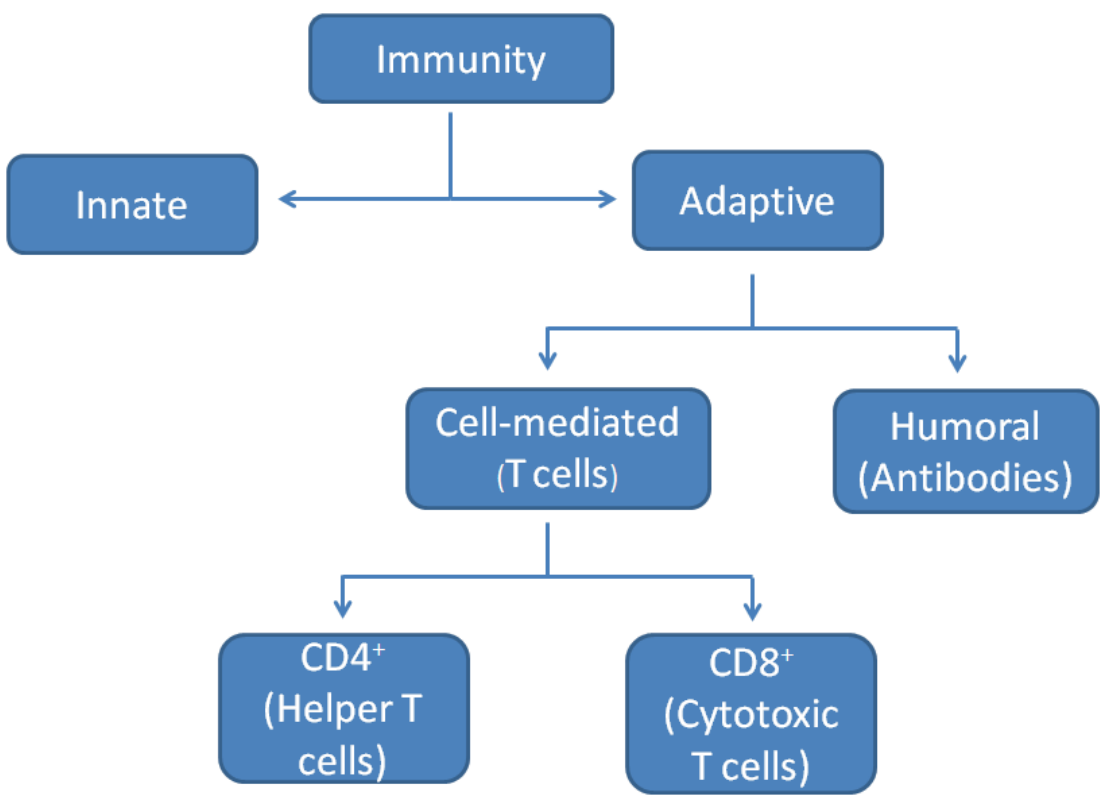

Figure 1-1. Types of immune response.

The immune response is divided into innate and adaptive immunity. Adaptive immunity is further classified into cell-mediated and humoral immunity. Finally, both $\mathrm{CD}^{+}$and $\mathrm{CD} 8^{+} \mathrm{T}$ cells are classified under cell-mediated immunity. 
(Fig. 1-2). In summary, the culmination of 3 signals leads to differentiation of naïve $\mathrm{T}$ cells into effector T cells.

Each major subset of Th cells requires different cytokines and transcription factors to regulate their differentiation. For Th1 cell differentiation, the master transcription factor T-box expressed in T-cells (T-bet) is required for differentiation and induction of IFN $\gamma$ [8]. Although the Th1 response is protective against intracellular pathogens, excessive uncontrolled Th1 response leads to tissue inflammation and autoimmune diseases. Although the name suggests that T-bet is only expressed in T cells, other immune cells such as Dendritic cells [9], NK, and NKT cells [10] can also express T-bet. During presentation to a naïve CD4 helper T cell, T-bet is induced by the synergistic effect of $\mathrm{T}$ cell receptor engagement and IFN $\gamma$-STAT1 signaling. Induction of T-bet expression stimulates expression of IFN $\gamma$ and IL-12 receptor inducible chain (IL-12R $\beta 2$ ) which makes the T cells responsive to IL-12 and induces another wave of T-bet expression stabilizing Th1 lineage [11-13]. The master transcription factor for Th2 cells is GATA3 which is induced by IL-4. The Th2 signature cytokines are IL-4, IL-5, and IL-13 which are required for eosinophil recruitment and IgG and IgE class switching. Th2 responses are important for extracellular pathogens and parasite clearance. IL-13 is involved in mucosal activation (mucus hypersecretion), and therefore an excessive Th2 response is associated with asthma [14].

The master transcription factor(s) for Th17 effector T cells are Retinoid-related Orphan Receptor (ROR) $\gamma t$ and ROR $\alpha$. The combination of proinflammatory cytokine IL-6 with immunoregulatory cytokine TGF $\beta$ stimulates Th17 cell differentiation in mice [15-18]. IL-6 and TGF $\beta$ cooperate in induction of ROR $\gamma$ t which is required for IL-17A and IL-17F mRNA expression [19]. The signature cytokine for Th17 cells is IL-17 which comes in several isoforms (IL-17A-IL-17F). In addition to IL-17, Th17 cells also secrete IL-21 and IL-22 which are important in the overall Th17 response to pathogens [19-22]. Several studies demonstrated the protective role of Th17 effector cytokines in extracellular and intracellular bacterial and fungal infections [23-28]. For instance, IL-17A and IL-17 receptor Knockout mice are prone to $K$. pneumoniae infection [27, 28]. Furthermore, Th17 cells are also protective in Mycobacterium tuberculosis infections indirectly, and IL-17 induces chemokines required for recruitment of protective Th1cells [24]. Also, in F. tularensis live vaccine infection model, IL-17 induces IL-12 from dendritic cells to establish a Th1 response and induces IL-12 and IFN $\gamma$ from macrophages to enhance bacterial killing [29]. Additionally, in acute respiratory tract infection caused by Mycoplasma pneumoniae IL-17A and IL-17F derived from Th17 cells are important in induction of lung neutrophil infiltration for pathogen clearance [25]. IL-22 also plays a protective role in $K$. pneumonia and $C$. rodentium mouse infection model [28, 30]. In $K$. pneumonia mouse infection model, neutralization of IL-22 increases the mortality rate compared to WT mice. Also, mice treated with anti-IL-22 had a significant increase in lung and spleen CFUs compared to WT mice [28]. Similarly, IL-22 KO mice infected with $C$. rodentium are characterized by a significant decrease in survival rate compared to WT mice [30]. In fungal infections, IL-17R A knockout mice are prone to Candida albicans infection compared to their wild-type counterparts and the injection of IL-17A into knockout mice protected them from lethal doses of infection [23]. Although Th17 


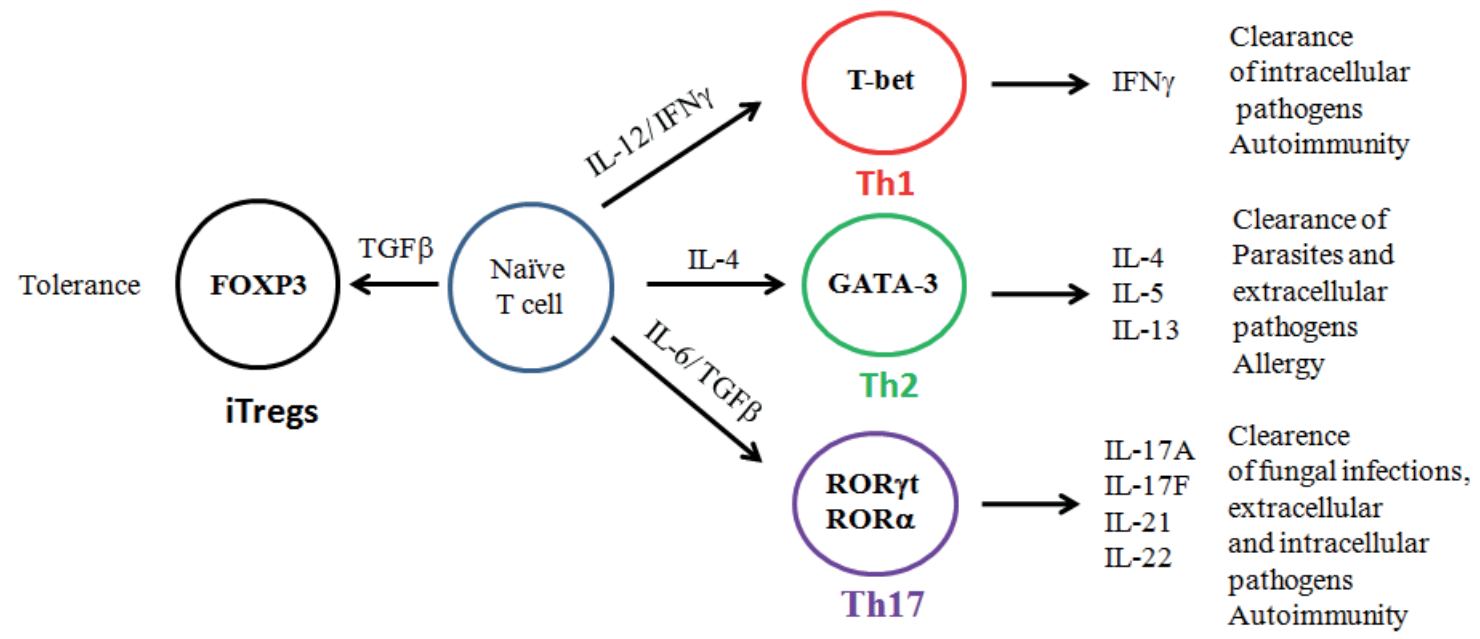

Figure 1-2. Differentiation of naïve $\mathrm{T}$ cell to different subsets of effector $\mathrm{CD} 4^{+} \mathrm{T}$ cells.

Naïve $\mathrm{CD}^{+} \mathrm{T}$ cells require three signals to differentiate into effector $\mathrm{CD} 4^{+}$helper $\mathrm{T}$ cells. Signals 1 and 2 come from $T$ cell receptor and costimulatory receptors signaling. The nature of the cytokine environment (signal 3) determines the fate of naïve $\mathrm{CD}^{+}{ }^{+} \mathrm{T}$ cells. IFN $\gamma$ and IL-12 are required for Th1 differentiation, while IL-4 is required for Th2 differentiation. The combination of IL- 6 and TGF $\beta$ are required for Th17 cell differentiation while TGF $\beta$ is required for differentiation of naïve T cells to iTregs (induced Tregs). Each subset of effector T helper cells has its own specific transcription factor. T-bet, GATA3, ROR $\gamma$ t and ROR $\alpha$ and Foxp3 are specific for Th1, Th2, Th17 and iTregs respectively. The effector $\mathrm{CD}^{+} \mathrm{T}$ cells are characterized by their signature cytokines. IFN $\gamma$ is the hall mark cytokine for Th1 cells while IL-4, 5, and 13 are specific for Th2. IL-17A, IL-17F, IL-21, and IL-22 are specific for Th17. (Adapted with permission from Bettelli, E., et al., Induction and effector functions of T(H) 17 cells. Nature, 2008. 453(7198): p. 1051-7 [31].) 
cells and cytokines play a protective role against numerous infections, several studies demonstrated their pathogenicity as inducers of tissue inflammation in a number of organ-specific autoimmune diseases such as Rheumatoid Arthritis, Multiple Sclerosis, and inflammatory bowel disease [32-38]. In conclusion, Th17 cytokines protect against a wide range of bacterial and fungal infections; however, Th17 cells can also play a pathogenic role in certain circumstances.

Regulatory T cells (Tregs) play a crucial role in maintaining immunological homeostasis and suppressing immune response. They are divided into two subsets, Natural Tregs (nTregs) that develop in the thymus and induced Tregs (iTregs) derived from peripheral naïve $\mathrm{CD}^{+} \mathrm{T}$ cells under the effect of TGF $\beta$ and IL-2. Foxp3 is the master transcription factor for Tregs and it is required for their suppressive function. Both cytokines (IL-2 and TGF $\beta$ ) are required for maintenance of Foxp3 in Tregs and iTregs. Mutations in Foxp3 causes a rare autoimmune disease IPEX (Immune-dysregulation Polyendocrinopathy Enteropathy X-linked) in humans characterized by a variety of inflammatory lesions in different tissues with massive production of Th1 and Th2 cytokines. Tregs use IL-2Ra (CD25) as a receptor for IL-2. Although they don't produce IL-2, they require IL-2 for their survival and expansion. They inhibit effector function of T cells through several mechanisms, e.g., suppression by cytokines, cytolysis, metabolic disruption, and targeting dendritic cells [14, 39-41].

\section{GRANULOMA FORMATION}

\section{Definition}

A granuloma is an organized mass of immune cells that mainly consists of macrophages, dendritic cells, B cells, and T cells. Even though a granuloma is an organized structure of cells, it is a dynamic microenvironment where immune cells are recruited to or egressing from the granuloma under the influence of chemokines and adhesion molecules. In addition, there is also cross-talk between the cells in the granuloma resulting in activation of cells and cytokine secretion. The main function of a granuloma is to wall-off the inciting agent from the surrounding tissue; however, if the microorganism is persistent and the granulomas do not resolve, tissue damage and eventually fibrosis occur giving granuloma characteristic of a double-edged sword [42-44].

\section{Requirements}

Although granuloma consists of different types of immune cells, several studies demonstrated the pivotal role of $\mathrm{T}$ cells in granuloma formation. RAG KO mice (mice deficient in $\mathrm{T}$ and $\mathrm{B}$ cells) were unable to form liver granulomas around schistosome ova and were unable to control bacterial dissemination or growth after mycobacterium infection. Furthermore, adoptive transfer of $\mathrm{CD}^{+}{ }^{+} \mathrm{T}$ cells into RAG KO mice infected 
with Schistosoma or mycobacterium causes granuloma formation [45]. Several studies have shown the crucial role of $\mathrm{CD} 4{ }^{+} \mathrm{T}$ cells in granuloma formation. HIV patients (patients with low CD4 T cells counts) are vulnerable to intracellular pathogens that require granuloma formation, such as M. tuberculosis, H. capsulatum, and Pneumocystis carinii [46]. In the Tubercule bacilli (TB) mouse infection model, depletion of $\mathrm{CD} 4^{+} \mathrm{T}$ cells leads to disorganized granuloma that results in reactivation and dissemination of tuberculosis [47]. In the same line of evidence, mice lacking functional $\mathrm{CD} 4^{+} \mathrm{T}$ cells are rendered susceptible to listeria infections which require granuloma for protection [48]. Additionally, depletion of $\mathrm{CD}^{+} \mathrm{T}$ cells in mice infected with Schistosoma mansoni developed smaller granulomas and less fibrosis [49]. Intuitively, the repertoire of T cells in the granuloma should be specific only to the antigen found at the site of inflammation; however, there are a percentage of non-specific $\mathrm{T}$ cells recruited into the granuloma. For example, in a $S$. mansoni mouse infection model, infection with LCMV (Lymphocytic Choriomeningitis Virus) causes recruitment of LCMV-specific CD8 ${ }^{+} \mathrm{T}$ cells into S. mansoni egg granuloma [50]. In summary, these studies have shown the importance of $\mathrm{T}$ cells specifically $\mathrm{CD} 4^{+} \mathrm{T}$ cells in granuloma formation.

Cytokines and chemokines are also considered important requirements for granuloma formation. Using a TB mouse infection model, several studies have shown the importance of TNF $\alpha$ and IFN $\gamma$ in the process of granuloma formation. The absence of $\mathrm{TNF} \alpha$ signaling causes a decrease in the number and size of granulomas [51, 52]. Also, there is an increase in the mortality of TNF $\alpha$ KO infected mice compared to WT infected mice [53]. Also, IFN $\gamma \mathrm{KO}$ mice fail to form granulomas following M. tuberculosis infection [54]. In conclusion, both TNF $\alpha$ and IFN $\gamma$ are important cytokines for granuloma formation. It difficult to distinguish the role of TNF $\alpha$ whether in the initiation or maintenance of granuloma since tissues were harvested at different time points following TB infection. To get around this limitation, Clay et al. used an intravital imaging system to track Mycobacterium marinum infected macrophages in zebra fish embryos deficient in TNF $\alpha$ signaling [55]. The authors found normal recruitment of macrophages to the site of infection in both WT and TNF $\alpha$ deficient zebra fish embryos suggesting that TNF $\alpha$ is dispensable for granuloma formation. The next crucial experiment was to determine if TNF is important for granuloma maintenance. Studies using a chronic TB mouse infection model showed that neutralization of TNF $\alpha$ leads to inability to maintain granuloma in the lungs [56, 57]. Furthermore, intravital imaging was used in mice exposed to Mycobacterium bovis BCG. TNFa neutralization caused a decrease in the size of granulomas since the trafficking of uninfected macrophages was reduced [58]. In conclusion, TNF $\alpha$ plays a crucial role in granuloma maintenance, but how does TNF $\alpha$ regulate granuloma maintenance? In one study, in vitro and in vivo neutralization of TNF $\alpha$ downregulated chemokines secreted from macrophages and other myeloid cells that may affect cell migration in and out of granuloma [56]. In conclusion, both TNF $\alpha$ and IFN $\gamma$ are important cytokines for granuloma formation. 


\section{Granuloma as a Dynamic Environment}

As mentioned before, granulomas are dynamic microenvironments where there are immune cells infiltrating and egressing the granuloma. The number of cells in the granuloma is determined by many factors. The balance between egression and infiltration of immune cells is one and survival time, proliferation, and death of the immune cells are other factors [42]. There are controversies regarding the role of TNF $\alpha$ in regulation of cell death. Several studies showed that blockage of TNFo in murine models caused macrophage apoptosis and major histopathological changes in the lung $[52,59]$. In contrast, in vitro TNF $\alpha$ induces apoptosis of human alveolar macrophages infected with M. tuberculosis [60]. Using the Zebrafish M. muranium infection model, there was an increase in granuloma non-apoptotic cell death in the absence of TNF signaling [55]. In conclusion, $\mathrm{TNF} \alpha$ plays a controversial role in regulation of cell death in granuloma.

The interaction between T cells and antigen presenting cells (APCs) is another process that dictates the microenvironment in the granuloma. For example, DCs with high expression of costimulatory molecules, MHCII and secretion of IL-12 were able to induce IFN $\gamma$ from Th1 cells which ultimately stimulates macrophages to kill TB, while IL-10 secreting DCs with low costimulatory and MHCII expression were not able to induce IFN $\gamma$ from $\mathrm{T}$ cells supporting TB persistence within macrophages [44]. In conclusion, the combination of several processes are required for granuloma formation; the balance between egression and recruitment of cells, cytokines and chemokines, survival of the cells within granuloma, and finally the cross-talk between the cells in the granuloma.

\section{Fate of Granuloma}

The fate of a granuloma may be resolution by apoptosis or alternatively a failure to resolve which may lead to fibrosis. Fibrosis is an uncontrolled wound repair process in which damaged tissue is replaced by a fibrotic scar that ultimately impairs organ function. The process is characterized by excessive production and accumulation of extracellular matrix (ECM) proteins (e.g. collagen, proteoglycans, and fibronectins) from myofibroblasts. It is a complex process that includes immune cells ( $\mathrm{T}$ cells, macrophages, and neutrophils), non-immune cells (epithelial cells, fibroblasts, myofibroblasts, and fibrocytes), and proinflammatory and profibrotic mediators. TGF $\beta$ is one of the profibrotic cytokines that plays a central role in fibrosis, it induces fibroblast proliferation and activation, production of ECM from myofibroblasts, and epithelial-mesenchymal transition process (EMT), a process in which epithelial cells transform into fibroblast-like cells [61]. Using bleomycin and farmer's lung models, studies have shown that IL-17A, and IL-17RA are involved in the process of pulmonary fibrosis in rodents [62] [63]. IFN $\gamma$ is considered an anti-fibrotic cytokine. In addition to being a transcription factor for Th1 differentiation T-bet is also a transcription factor for induction of IFN $\gamma$ in particular cells. Numerous studies have also investigated the role of T-bet in fibrosis. T-bet KO mice treated with bleomycin showed an increase in lung collagen content compared to WT mice [64]. Furthermore, in the absence of T-bet mice had increased hydroxyproline in the 
skin following bleomycin treatment compared to WT counterparts [65].

\section{HYPERSENSITIVITY PNEUMONITIS}

\section{History}

In 1713, B. Ramazzini, an Italian medical professor, observed the health hazards in grain workers and stated "Almost all who make a living by sifting or measuring grain are short of breath and cachectic and rarely reach old age..." [3, 66]. With this description he gave the first account for what is now known as Hypersensitivity Pneumonitis (HP). In the $20^{\text {th }}$ century, several published reports described outbreaks of respiratory diseases that were later defined as HP. In 1932, the initial clinical description of HP was developed following an outbreak of respiratory illness in a Michigan Company. The workers were stripping bark from maple logs and developed symptoms such as dyspnea, coughing, weight loss, and sputum. Similar symptoms appeared in labors working with maple logs at a nearby sawmill. At both locales, investigators found black dust lying beneath the bark from which they isolated a fungus called Cryptostroma corticale [67]. The authors hypothesized that the symptoms resulted from an allergic reaction to the spores of this microorganism. Another outbreak of respiratory disease was reported among agricultural workers who also developed weight loss and dyspnea in Northern England [68]. Once the workers ended their contact with hay, their symptoms disappeared within several weeks. Twelve years later, similar conditions were recognized and reported by W.N Pickles, a Yorkshire clinician. He referred to the condition as farmer's lung [66].

\section{Different Types and Etiological Agents of HP}

Hypersensitivity pneumonitis (HP) is an interstitial lung disease caused by repeated inhalation of environmental antigens resulting in alveolitis, granuloma formation and sometimes fibrosis [3, 69-71]. There are a wide range of environmental agents that cause HP and they include animal proteins, fungi, and bacteria (Table 1-1). Each type of HP is named after the antigen, occupation, or hobby performed by the exposed individual [72]. For example, Farmer's Lung Disease (FLD) is a classic example of HP caused by repeated exposure to thermophilic bacteria called Saccharopolyspora rectivirgula (SR) [73]. SR (rectus $=$ straight and virgula=twig) is an aerobic thermophilic gram positive spore-forming bacterium that belongs to the actinomycetes family. It has a type IV cell wall which contains meso diaminopimelic acid (meso-DAP), galactose, and arabinose. The bacteria grow in moldy hay as well as soil, compost, and manure [74]. When the hay is disturbed, the spores become airborne and inhaled by farmers. In eastern France, other organisms such as fungal species A. corymbifera, E. amstelodami, and $W$. sebi were isolated from cowshed air and fodder and identified as potential etiological agents for FLD in the absence of SR [75]. Different farming practices affect fungal and bacterial concentration in hay and subsequently the disease risk [76]. Other types of HP include 
Table 1-1. Different forms of HP showing putative antigens and their source.

\begin{tabular}{ccc}
\hline Disease & Antigen source & Antigen \\
\hline Bird fancier's lung & Pigeon and parakeet & $\begin{array}{c}\text { Avian proteins } \\
\text { in feces and feathers }\end{array}$ \\
Bagassosis & Moldy cane sugar & $\begin{array}{c}\text { Thermophilic } \\
\text { actinomycetes }\end{array}$ \\
Cheese worker's lung & Moldy cheese & Penicillium casei \\
Farmer's lung & Moldy hay & Thermophilic \\
Hot tub lung & Contaminated warm water & Mycobacterium avium \\
Malt worker's lung & Moldy malt & Aspergillus sp. \\
Maple bark disease & Maple logs & Cryptostoroma corticle \\
Machine worker's lung & Metal-cutting fluid & $\begin{array}{c}\text { Mycobacterium sp. and } \\
\text { Gram-negative Bacilli }\end{array}$ \\
Tobacco worker's lung & Moldy tobacco & Aspergillus sp. \\
Peat moss worker's & Moldy peat moss & Penicillium sp. \\
Suberosis & Moldy cork & Aspergillus sp. \\
\hline
\end{tabular}

(Adapted with permission from Hirschmann, J.V., S.N. Pipavath, and J.D. Godwin, Hypersensitivity pneumonitis: a historical, clinical, and radiologic review.

Radiographics, 2009. 29(7): p. 1921-38 and Dhar, S. and F. Daroowalla, Hypersenitivity Pneumonitis. Clinical Pulmonary Medicine, 2011. 18(4): p. 169-174 [66, 77].) 
Bird Fancier's Lung, which results from exposure to avian proteins found in feathers, serum, or bird droppings [78]. Fungi are another source of HP antigens. For example, members of the Aspergillus species induce disease among corn and malt workers [79, 80]. Also cheese, cork, and peat moss workers develop HP due to repeated exposure to Penicillium species [81-83]. The enzyme phytase that is added to cattle feed and, also molds from dry sausage have been described as a potential etiological agent for HP [84, 85]. Despite the wide range of etiological agents, all HP types are characterized by alveolitis, and granuloma formation; some patients develop fibrosis, which is associated with significant morbidity and mortality [86].

\section{Classification, Clinical Diagnosis, and Epidemiology}

\section{HP Classification}

HP can be classified into acute, subacute and chronic forms [87]. Acute HP is the most common clinical presentation and is characterized by flu-like symptoms including chills, malaise, fever and myalgias. Other pulmonary symptoms, including a nonproductive cough, chest tightness, or dyspnea may accompany the acute form. The symptoms begin 2-9 hours after exposure and peak 6-24 hours after exposure then resolve without any treatment 1-3 days post exposure cessation [88]. The subacute form of HP is mainly characterized by a progressive cough, dyspnea, fatigue, weight loss, and granuloma formation. The symptoms develop with continuous exposure and occur within weeks to a few months of exposure $[70,89,90]$. The hallmark of chronic HP is progressive, irreversible pulmonary fibrosis that takes months to years to develop, with a mortality rate $1-10 \%$ [3]. There are conundrums surrounding this classification since there is a lot of overlap between HP stages and no accepted criteria to distinguish between the three stages. Recently, the classification of HP was revisited in a study [91]. The authors took advantage of a multicenter cohort HP study done between February 1998 and September 2001 from seven clinical sites in different countries and performed cluster analysis to compare them to the current classification, i.e., acute, subacute, and chronic [92]. The results from the cluster analysis did not confirm the current classification, thus suggesting that HP patients are divided into two clusters. The first cluster of patients develop chest tightness after antigen exposure, body aches, and chills, with normal chest radiographs, while the second cluster is characterized by restrictive pulmonary function tests, resting hypoxemia, and inspiratory crackles. One third of this cluster develop fibrotic changes, as seen on High Resolution Computed Tomography (HRCT) [91]. In conclusion, the current classification was not verified by this study in which HP patients were classified into two categories rather than three; however, the authors mentioned that their classification requires further validation.

\section{HP Clinical Diagnosis}

There is no solid standard diagnostic test to rule out HP from other interstitial lung diseases [77]; however, a study proposed six diagnostic criteria for HP and when all six factors are present the probability of HP is $98 \%$. The six criteria are as follows: 
(1) Exposure to a known inciting agent, (2) loss of weight, (3) detection of precipitating antibodies from patient sera, (4) on physical examination, inspiratory crackles are present, (5) symptoms occur four to eight hours after exposure, and (6) the patient experiences recurrent episodes of symptoms [92]. Chest X-ray is usually the first step in the diagnosis of a patient with pulmonary disease. It mainly rules out other pulmonary diseases rather than HP. Up to $20 \%$ of patients with acute HP have normal X-rays [93].Usually the X-ray findings are not specific for HP showing the non-specificity of this approach. Another diagnostic approach is the detection of serum-precipitating antibodies. Several studies indicated that the presence of serum-precipitating antibodies doesn't really reflect the occurrence of HP. For instance, it has been reported that up to $50 \%$ of pigeon breeders and $30 \%$ of farmers develop serum-precipitating antibodies with no evidence of disease symptoms $[94,95]$. Furthermore, $78 \%$ of subjects exposed to known antigens that cause HP had serum-precipitating antibodies while $38 \%$ of the control group subjects were diagnosed with serum-precipitating antibodies [92].There are several factors that contribute to the failure to detect serum-precipitating antibodies in a given patient, they include usage of poorly purified antigens, lack of specificity, and diluted serum patient samples [96]. The antibody concentration in the serum is low in HP patients with smoking history [97-99]. In summary, serum-precipitating antibodies are good markers against specific antigens; however, their absence does not negate diagnosis with HP. Lung function tests are nonspecific for HP since the lung function changes are similar to other interstitial lung diseases [90]. In general all forms of HP display restrictive pattern of lung function, including reduced lung volumes and impairment in $\mathrm{O}_{2}$ transfer from airspace to blood (Diffusing Capacity of Lung for Carbon monoxide, $\mathrm{DL}_{\mathrm{co}}$ ), reduced compliance (the ability of the lungs to stretch back to their original size after removal of a compressing force), and hypoxemia induced by exercise [100]. Analysis of the cellular composition of bronchoalveolar lavage (BAL) from patients is another tool to diagnose HP. During BAL a saline solution is injected into the lungs and then retrieved back to be examined by flow cytometry. In normal healthy subjects, $90 \%$ of the retrieved cells are alveolar macrophages and the rest are lymphocytes, neutrophils and eosinophils [101]. During an acute HP episode neutrophils are the main cell population in the BAL within 48 hours of an exposure [102] which become lymphocytic later on.

The most effective way to manage HP is identifying the causative agent and avoiding exposure to it; however, it is difficult to identify the inciting agent and adding to that exposure cessation might put the patient's daily work at risk. Complete cessation of exposure may help acute HP patients to experience a reversal of symptoms, but once the disease reaches the fibrotic stage it becomes irreversible. This results in significant decline in lung function and can lead to death even if patients avoid exposure to the inciting agent. Although corticosteroids are another option to improve lung function; it does not replace the necessity of antigen identification and contact withdrawal $[3,77]$.

\section{HP Epidemiology}

The incidence and prevalence of HP are variable and hard to determine for several reasons. The methods used to diagnose HP are limited, making HP often misdiagnosed as viral pneumonitis or idiopathic interstitial disease [90]. Also, most HP studies were 
cross-sectional, while large scale surveys were few and mainly dependent on questionnaire and detection of serum-precipitating antibodies [77, 103]. As mentioned above, the presence of antibodies against specific antigen doesn't rule out HP. The issue gets more complicated by geographical, climatic conditions and farming practices. Population studies have reported varying rates of HP incidence. In New Mexico, the incidence of HP between 1988-1990 was 0.6 per 100,000 cases, whereas the incidence of HP in Wisconsin dairy farmers was 42 per 100,000 [104, 105]. In the Czech Republic, the most common types of HP were Farmer's lung, Malt worker's lung and Chemical worker's lung; however the incidence between 1992 and 2005 was only 0-0.2 per 100,000 workers[106]. In the UK, the most common types of HP were found in the agriculture, fishing, and forestry industries and the incidence 1992 and 2001 was $1.6 \%$. [107]. Aside from the environmental factors and farming practices, genetic factors add another layer of complexity in the epidemiology of HP. For example, certain alleles of Major Histocompatibility Complex, TNF $\alpha$ promoter, and LMP2 and 7 (encode subunits in the proteosome complex for degradation of cytosolic proteins and generation of antigenic peptides) are associated with Pigeon breeder's disease [108, 109]. In conclusion, combination of several factors is responsible for the variability in HP incidence and prevalence.

\section{Immunopathogenesis}

Immunopathogenesis of HP in Patients

Despite the fact that a wide range of environmental agents may cause HP, the disease is characterized by alveolitis, granuloma formation and fibrosis. In HP patients, one of the hallmarks of the acute stage is neutrophilic alveolitis [102]. During acute HP, the patients were diagnosed with a significant increase in the BAL cell numbers as well as the percentage of neutrophils compared to control subjects [102]. Although CXCL2 (IL-8), a neutrophil chemokine, was detected in the BALF of HP patients $[110,111]$, the source was not identified. Later on alveolar macrophages were discovered as the potential source of IL-8 in HP patients [112]. The recruitment of neutrophils into the air space of the lungs causes tissue damage through production of proteases and oxygen free radicals. In patients with lung fibrosis, there was an increase in the number of lung neutrophils as well as increase of matrix metalloproteinases (MMPs), MMP-8 and 9 compared to control subjects [113]. The correlation between fibrosis and production of MMPs by neutrophils suggested the role of neutrophils in tissue remodeling and eventually lung fibrosis [113]. In conclusion, the previous studies suggested that AMs initiate inflammatory environment in the air space by recruitment of neutrophils through IL-8 and they play a pathogenic role during fibrosis stage in HP.

CXCL2 is not the only chemokine produced by AMs. AMs isolated from HP patients spontaneously produce an array of cytokines and chemokines, which eventually contribute to macrophage and $\mathrm{T}$ cell recruitment and granuloma formation. For instance, in acute and chronic HP patients, AMs produce increased amounts of IL-12, IL-18, and TNF $\alpha$ without ex vivo stimulation compared to control subjects [114]. IFN $\gamma$ stimulates 
macrophages to produce TNF $\alpha$ and IL-1 $\beta$ which further stimulates macrophages to produce additional chemokines recruiting monocytes to the lungs for granuloma formation [115]. Furthermore, IFN $\gamma$ inducible chemokine CXCL10 is produced from AMs isolated from HP patients. Consequently, CXCL10 recruits $\mathrm{T}$ cells bearing the CXCR3 receptor especially $\mathrm{CD} 8^{+} \mathrm{T}$ cells into the lungs [116]. Additionally, CCL3/MIP-1 $\alpha$, chemokine that contributes to recruitment of $\mathrm{CD}^{+} \mathrm{T}$ cells, was detected in the BALF from HP patients [111]. Several studies demonstrated that $\mathrm{CD}^{+} \mathrm{T}$ cells were the predominant T cells in the BAL of HP patients with acute and subacute stages, decreasing $\mathrm{CD}^{+} / \mathrm{CD}^{+}$ratio; however, in the fibrosis stage $\mathrm{CD} 4^{+} \mathrm{T}$ cells were elevated increasing the $\mathrm{CD} 4^{+} / \mathrm{CD} 8^{+}$ratio. These ratios could change based on the type of the antigen [117-120]. Although AMs are considered as poor antigen presenting cells, AMs isolated from HP patients are characterized by upregulation in HLA DR (MHCII) which binds to the T cell receptor (TCR), co-stimulatory molecules (CD80 and CD86) which bind to CD28 on the T cells, and Intracellular adhesion molecule-1 (ICAM-1) which binds to Lymphocyte Function Associated antigen-1 (LFA-1) on T cells increasing adhesion for T cell binding [121-123]. All these characteristics suggest they may be important for $\mathrm{T}$ cell activation. In conclusion, AMs appear to contribute to production of a wide range of cytokines and chemokines that create a proinflammatory environment leading to recruitment of different immune cells and eventually granuloma formation.

Immunopathogenesis in an Experimental Animal Model

Although the previous HP studies can give us an appreciation for how disease complexity, the studies are very limited regarding the number of samples and the techniques used. Therefore, several animal models have been used to unravel the intricate mechanisms behind the disease. The SR mouse model of HP mimics Farmer's lung disease, and in this model C57BL/6J mice are repeatedly intranasally exposed to SR. Following one exposure there is a neutrophilic alveolitis that peaks at 24-48 hours followed by lymphocytosis at 48-72 hours. Bronchoalveolar lavage fluid (BALF) is characterized by an increase in proinflammatory cytokines and chemokines such as IL-6, TNF $\alpha$, IL-1 $\alpha$, CCL2 and CCL3 [124]. With continued exposures for 3 days per week for three weeks the alveolitis becomes increasingly lymphocytic and there is the formation of peribronchiolar granulomas [103]. The development of fibrosis with chronic exposures in this model was an advantage for several studies to use and understand the role of different cells and mediators in fibrosis during HP [62, 125, 126].

The wide range of proinflammatory cytokines and chemokines found in the BALF could be produced by different types of cells in the lung following SR inhalation. Alveolar macrophages might be potential candidates for production of these mediators. In vitro stimulation of macrophages cell line (J744A.1) and murine alveolar macrophages (AMs) with SR resulted in secretion of different cytokines and chemokines such as CCL2, CCL3, IL-6, IL-12p40, TNF $\alpha$, and IL-1 $\alpha$ [124]. The combination of these mediators create a proinflammatory environment in the airspace. One of the consequences of a proinflammatory environment in the airspace is recruitment of immune cells: neutrophils, monocytes, and T cells. Early neutrophilic influx into the airways is one of the hallmarks of acute HP [102]. CXCL2 (mouse homologue for IL-8) 
was detected in the BALF of C57BL/6 mice following SR exposure; however, it was significantly lower in the BALF of MyD88 KO mice (Adaptor protein downstream TLRs) [127]. In the same line of evidence, SR stimulation of WT adherent spleen cells in vitro induces CXCL2 production while MyD88 KO produces significantly less amount of CXCL2 protein [127]. In summary, SR induces production of proinflammatory mediators from lung cells e.g. alveolar macrophages resulting in a proinflammatory environment and recruitment of immune cells into the lungs and airspace.

The continuous exposure to SR leads to recruitment of T cells and eventually granuloma formation which is IFN $\gamma$ dependent during HP [128]. However, the cellular source of IFN $\gamma$ was not identified at that period of time. Nance et al. identified neutrophils as an important source of IFN $\gamma$ required for granuloma formation [129]. IFN $\gamma$ induced recruitment of $\mathrm{CXCR}^{+} / \mathrm{CD}^{+} \mathrm{T}$ cells through induction of IFN $\gamma$ inducible chemokines I-TAC, MIG, and IP-10 during HP [130]. Furthermore, IFN $\gamma^{-/-}$mice exposed to SR did not develop granulomas while WT counterpart did, demonstrating the importance of IFN $\gamma$ in granuloma formation during HP [128]. Since IL-10 (anti-inflammatory cytokine) antagonizes IFN $\gamma$ biological actions, the role of IL-10 was also examined during HP. Following three weeks of SR exposure, IL-10 KO mice developed more alveolitis and lung inflammation compared to WT exposed mice. Also, IL-10 KO mice were characterized by up regulation in the gene expression of TNF $\alpha$, IL-1, and IFN $\gamma$ in the lungs compared to WT exposed mice. The replacement of IL-10 in IL-10 KO exposed mice dampened the inflammation compared to IL-10 KO mice without IL-10 replacement [131]. In summary, these data demonstrated that IFN $\gamma$ and IL-10 play a crucial role in the development of HP.

As described above various cytokines and chemokines were produced generating a proinflammatory environment. TNF $\alpha$ is one of the proinflammatory cytokines that plays a crucial role in various biological activities during inflammation, e.g. AMs' and neutrophils' activation and stimulation of the endothelium. The role of TNF $\alpha$ during HP was examined [126, 132-134]. Following three weeks of SR exposure, mice treated with anti-TNF $\alpha$ were characterized by less inflammation in the airways and lung, down regulation of IL-1 in BALF, and decreased collagen deposition in the lungs compared to mice treated with control rabbit serum [126]. The decrease in lung collagen following anti-TNF $\alpha$ suggested inhibition of TGF $\beta$ (profibrotic cytokine that induce collagen production) during HP. Consequently, levels of TGF $\beta$ and collagen content were tested in the lungs of C57BL/6 mice during HP. There was a significant increase in the levels of lung TGF $\beta$ following one week of SR exposure and increase in collagen content following two weeks of SR exposure compared to saline exposed mice. Furthermore, in vitro stimulation of alveolar macrophages from SR exposed mice produced significant amounts of TGF $\beta$ compared to AMs from saline exposed mice [125]. In conclusion, both TNF $\alpha$ and TGF $\beta$ are required for development of HP. 
More than 40 years ago, HP was considered as an antibody-mediated disease (Type III hypersensitivity reaction) in which the inhaled environmental antigen binds to an antibody, forming antigen-antibody immune complexes. The deposition of these immune complexes in the lung initiate the disease through activation of classical complement pathway and alveolar macrophages secreting inflammatory cytokines; hence, lung inflammation is initiated as discussed above. [99, 103]. However, several observations during HP were not supported by this idea. First, granuloma formation and lymphocytic bronchoalveolar lavage (BAL) support the notion that HP is indeed a cell-mediated disease (Type IV hypersensitivity pneumonitis). Second, lack of vasculitis (a hallmark of Type III hall marks) and failure to produce lesions similar to human patients upon passive transfer of hyper immune serum [86, 103]. Third, the disease may occur in individuals lacking antibodies [135]. Finally, asymptomatic individuals may also develop antibodies aganist HP antigens [95]. In conclusion, these observations support the importance of type IV hypersensitivity reaction over type III hypersensitivity reaction during HP.

The discovery that humoral immunity (antibody mediated) does not play an important role in HP pathogenesis led the HP research field to change its view toward the disease and start to investigate the other arm of adaptive immunity, which is cellular immunity. Numerous studies have demonstrated the importance of $\mathrm{T}$ cells in the pathogenesis of the disease. C57BL/6 athymic nude mice (nu/nu), which lack T-cells, develop less lung inflammation compared to $(+/ \mathrm{nu})$ mice when exposed to Thermoactinomyces vulgaris, an HP causative agent [136]. To determine the role of $\mathrm{T}$ cells in this model, nu/nu mice were injected with splenic $\mathrm{T}$ cells obtained from sensitized + /nu-mice. The results demonstrated that the recipients develop lung inflammation similar to + /nu-exposed mice, suggesting that $\mathrm{T}$ cells play a pathogenic role [136]. However, it was unknown whether $\mathrm{CD} 4^{+}$or $\mathrm{CD} 8^{+} \mathrm{T}$ cells were responsible for the disease. Schuyler et al. demonstrated that adoptive transfer of $\mathrm{CD}^{+}{ }^{+} \mathrm{T}$ cells but not $\mathrm{CD}^{+} \mathrm{T}$ cells from spleens or lung lymph nodes of SR sensitized mice into naïve mice resulted in lung inflammation [137]. Similarly, adoptive transfer of $\mathrm{CD}^{+}{ }^{+} \mathrm{T}$ cells into $\mathrm{TCR} \beta \delta$ double knockout mice (mice deficient in $\mathrm{CD}^{+}, \mathrm{CD}^{+}$and $\gamma \delta \mathrm{T}$-cells) resulted in lung inflammation and pulmonary fibrosis [62]. Finally, adoptive transfer of splenic cells treated with anti-CD $4^{+}$antibody from SR sensitized mice failed to transfer the disease to naïve animals [138] confirming the pathogenic role of $\mathrm{CD}^{+} \mathrm{T}$ cells during HP. In conclusion, $\mathrm{CD}^{+}{ }^{\mathrm{T}}$ cells are pathogenic in HP.

Role of Th1 Cells and IFN $\gamma$ during HP

Initial studies suggested that HP was a Th1 cell-mediated disease rather than a Th2 cell-mediated disease. Th1 biased C57BL/6 mice are susceptible to HP while the Th2 biased DBA/2 mice are resistant to HP [139, 140]. Also, GATA-3 knock-in transgenic mice develop a less severe form of HP than their WT counterparts [141]. Additionally, based on pulmonary histological changes, it was found that CD4 ${ }^{+} \mathrm{T}$ cells that adoptively transfer HP are Th1 but not Th2 cells [142]. Finally, knocking out 
IFN $\gamma$, the Th1 signature cytokine protects SR exposed mice from granuloma formation while WT SR exposed mice develop granuloma [128]. In conclusion, these studies suggested that Th1 cells were important for the disease and that IFN $\gamma$ was necessary for granuloma formation. However, it was not clear whether IFN $\gamma$ produced by Th1 cells is required for disease severity during HP. Additional studies by our lab demonstrated that adoptive transfer of T cells from IFN $\gamma$ KO mice to RAG-1 KO mice (mice deficient in $\mathrm{T}$ and $\mathrm{B}$ cells), followed by exposure to SR, resulted in the same level of disease severity as adoptive transfer of WT T cells. These results demonstrated that T cell IFN $\gamma$

production was not necessary for the disease pathogenesis and that IFN $\gamma$ production by innate immune cells was sufficient. Therefore, the development of a Th1 response may not be necessary for the development of the disease [129]. These data clearly demonstrate that IFN $\gamma$ produced by Th1 is not required for granuloma formation and a non-T cell IFN $\gamma$ source is sufficient for granuloma formation. Using intracellular cytokine staining, the innate immune source of IFN $\gamma$ was identified as neutrophils. Depletion of neutrophils causes down-regulation of IFN $\gamma$ at both the mRNA and protein levels, confirming the importance of neutrophils as a significant source of IFN $\gamma$ during HP [129]. In conclusion, the previous studies suggested that HP was a Th1-mediated disease; however, IFN $\gamma$ produced by Th1 cells is not necessary for granuloma formation. 


\section{CHAPTER 2. HP AND TH17}

While it was once thought that HP is a Th1 cell mediated disease, the results demonstrating that IFN $\gamma$ produced by Th1 cells was dispensable for granuloma formation suggested otherwise. Consequently, we examined the T cell response during HP in an attempt to determine whether a Th17 response rather than a Th1 response could be responsible for the disease. The aim of these studies is to determine whether a Th17 response developed in the mice during the granulomatous and chronic phases of HP.

\section{INTRODUCTION}

\section{Th17 Cell as a Distinct CD4 ${ }^{+}$T Helper Cell}

The past few years have witnessed a revolution in the field of $\mathrm{T}$ helper cell biology with the discovery of an additional effector T helper cell subset, Th17 cells. These cells are characterized by their own distinct transcription factors, (ROR $\gamma \mathrm{t}$ and ROR $\alpha$ ) and signature cytokines (IL-17A, IL-17F, IL-21, and IL-22) [19-22]. Th17 cell differentiation is induced by the combination of IL-6 and TGF $\beta$. [15-18]. Both cytokines cooperate together for induction of ROR $\gamma \mathrm{t}$ which is required for IL-17A and IL-17F mRNA expression [19]. To further demonstrate the importance of IL-6 and TGF $\beta$ in Th17 differentiation, studies have shown that absence of TGF $\beta$ receptor resulted in a defect in generation of Th17 cells, while TGF $\beta$ transgenic mice have a higher frequency of Th17 cells following induction of Experimental Autoimmune Encephalomyelitis (EAE, a mouse model for multiple sclerosis) $[18,143]$. Similarly, IL-6 KO mice lack Th17 cells; however, the dominant cell response is mediated by T regulatory cells (Tregs) showing the inhibitory effect of IL-6 on TGF $\beta$ driven Foxp3 expression. Nevertheless, depletion of Tregs in IL-6 KO mice restores Th17 cell differentiation, indicating the presence of another factor besides IL-6 for Th17 differentiation, IL-21 [144]. Since IL-21 is produced by Th17 cells, IL-21 in an autocrine manner, together with TGF $\beta$, is able to induce Th17 cell differentiation independently of IL-6 [144-146]. All the previous characteristics made Th17 classified as a distinct subset of $\mathrm{CD}^{+}$helper T cells.

IL-23 is a member of the IL-12 family of cytokines that consists of 2 chains, IL-23 p19 specific for IL-23 and the IL-12p40 that is common with IL-12 [147]. Since, IL-23R is not expressed by naïve T cells, IL-23 is not involved in Th17 differentiation; however, IL-23R is expressed on differentiated Th17 cells. In vitro data showed that IL-23 is required for IL-17 secretion from Th17 cells but not for their proliferation and commitment [148]. The role of IL-23 in several disease models was examined. For example, it was shown that IL-23p19KO mice were protected from induction of EAE [149]. Furthermore, Th17 cells cultured in vitro with IL-23 were able to transfer EAE $[150,151]$. These Th17 cells acquired pathogenic characteristics, whereas Th17 generated in presence of IL- 6 and TGF $\beta$ only were did not induce disease due to anti-inflammatory cytokine IL-10 production [152]. Recently, this idea was supported by 
a study showing that naïve T cells cultured in the presence of IL-6, IL-1 $\beta$, and IL-23 possess a pathogenic characteristic and able to transfer EAE to the mice via adoptive transfer while culturing in the presence of IL- 6 and TGF $\beta$ only made them nonpathogenic [153]. More than 2,000 genes were differentially expressed between both types of cells, showing that both have different expression profiles [153]. Although these studies demonstrated the importance of IL-23 for Th17 pathogenicity in an autoimmune disease model, the factor(s) downstream of IL-23 remained to be identified. Several studies demonstrated that Granulocyte/Macrophage Colony Stimulating Factor (GM-CSF) was produced by Th17 cells following IL-23 stimulation [150, 154, 155]. GM-CSF plays a pathogenic role in EAE. Neutralization of GM-CSF inhibits development of EAE and enhances the recovery [155]. Antigen presenting cells express the GM-CSF receptor whereas T cells do not and the main action of GM-CSF is to upregulate MHCII and proinflammatory cytokines, e.g., IL-6. In EAE, GM-CSF deficient mice have reduced MHCII expression on microglial cells [155] with less accumulation of macrophages into the CNS [156]. Also, GM-CSF was shown to stimulate IL-23 from dendritic cells, causing activation of Th17 cells and formation of IL-23-GM-CSF positive feedback loop [154]. To conclude, IL-23 plays a role in the pathogenicity of Th17 cells.

\section{Effector Function of Th17 Cytokines}

Although IL-17 is considered the signature cytokine of Th17, it is expressed by other immune cells [157-159]. IL-17 (also known as IL-17A) belongs to the IL-17 family of cytokines which includes IL-17B-IL-17F members. It plays a role in host defense and inflammation by induction of proinflammatory cytokines (IL-1, TNF $\alpha$, and IL-6), chemokines (CXCL1, CXCL2, CCL2, and CCL7), matrix metalloproteinases (MMP1, 3, and 13) and antimicrobial peptides (defensins and S100 proteins). Furthermore, IL-17 is involved in granulopoiesis and neutrophil recruitment by induction of G-CSF and neutrophil chemokines such as CXCL2 [160]. Previous studies demonstrated the correlation between IL-17 and airway neutrophil recruitment [161-163]. Administration of exogenous IL-17 induced neutrophil recruitment as well as upregulation in neutrophil chemokine, CXCL2 [161-163]. Following LPS intranasal administration, there was an increase in IL-17 protein expression in the BALF accompanied by an influx of neutrophils into the airways [162]. Moreover, IL-17 neutralization results in a decrease in the number of airway neutrophils and CXCL2 protein expression in BALF [162] and IL-8 secretion from 16HBE, human bronchoepithelial cells [161]. Taken together, these studies emphasize the importance of IL-17 in regulation of neutrophil recruitment into airways.

IL-22 is another cytokine secreted by Th17 cells, although it is also expressed by a wide range of innate and adaptive immune cells e.g. NK cells, NKT cells, $\gamma \delta$ T cells, dendritic cells, and CD8 T cells. The IL-22 receptor is expressed on nonhematopioetic cells lining barrier surfaces such as lung, intestine, and skin as well as liver and kidneys [164]. Several studies demonstrated the protective effect of IL-22 on airway and intestinal epithelium through induction of antimicrobial peptides and enhancement of epithelial barrier integrity $[28,30]$. Also, IL-22 induces expression of chemokines and 
cytokines CXCL1, CXCL5, CXCL9, IL-6, and G-CSF required for host defense against bacterial infection $[28,30]$. Furthermore, IL-22 induces wound healing by increasing cell migration in intestinal epithelial and keratinocyte in vitro wound assay $[165,166]$. IL-22 also stimulates expression of antiapoptotic molecules e.g. BcL-2, BcL-xL as well as protective mucin (muc1, muc3, muc10, and muc13) [164]. In a mouse model of hypersensitivity pneumonitis, blocking IL-22 increases collagen deposition in the lungs while injection of IL-22 prevents lung fibrosis. On the contrary, IL-22 promotes pathological effects as well. For example, IL-23 stimulates dermal inflammation in an IL-22 dependant fashion. In the same line of evidence, IL-22 KO mice were characterized by decreased inflammation and epidermal hyperplasia in psoriasis mouse model [167]. Furthermore, neutralization of IL-22 in psoriasis-like skin disease ameliorates the disease severity and decreases expression of antimicrobial peptides [168]. To conclude, the previous results demonstrated the pathological and protective role of IL-22 in different experimental animal models.

\section{Is HP a Th1/Th17-Mediated Disease?}

HP was once considered as a Th1 mediated disease for several reasons. Several studies demonstrated the importance of Th1 cells in HP, while other studies demonstrated the importance of the Th1 cytokine IFN $\gamma$. IFN $\gamma$ knockout mice (GKO) when exposed to SR did not develop granulomas compared to the WT mice [128]. Previous results from our lab demonstrated that T cell IFN $\gamma$ production was not necessary for the disease and that IFN $\gamma$ production by innate immune cells was sufficient. Therefore, the development of a Th1 response may not be necessary for the development of the disease [129]. However, since T cells are necessary for granuloma formation, is there is another CD4 ${ }^{+}$ T cell subset, such as Th17 or Th2 cells, required for disease severity? The objective of this chapter is to determine the T cell response (beside Th1 response) that develops during the granulomatous and chronic phases of HP.

\section{MATERIALS AND METHODS}

\section{Bacterial Preparation and Exposure Protocol}

\section{Bacterial Preparation}

SR was obtained from the American Type Culture Collection (strain designation A1313; ATCC, Rockville, MD). It is a thermophilic gram-positive bacterium formerly known as Micropolyspora faeni belonging to the Pseudonocardiacecae family. The bacterium was grown in Trypticase Soy Broth (BD; Sparks, MD) at $55^{\circ} \mathrm{C}$ in a shaking incubator then harvested by centrifugation at $2000 \mathrm{rpm}$ for 15 minutes and washed with sterile endotoxin free water three times followed by lyophilization. The lyophilized preparation was reconstituted at a concentration of $5 \mathrm{mg} / \mathrm{ml}$ in endotoxin-free saline and determined to be endotoxin free using the Limulus ambeocyte lysate assay (Lonza; 
Walkersville, MD). This preparation was used for intranasal exposures.

SR Exposure Protocol

Mice were anesthetized using isoflurane and SR $(50-225 \mu \mathrm{g} / \mathrm{ml})$ was inoculated intranasally in a total volume of $40 \mu \mathrm{l}$. To study the innate immune response following exposure to SR, mice were exposed once or twice and sacrificed 24 hours after the last exposure. Studies on granuloma formation and the adaptive immune response were performed after three weeks of exposure. Mice were intranasally exposed to SR on 3 consecutive days per week for three weeks (Fig. 2-1) and sacrificed 18 hours after the last exposure (Day 18) or 4 days after the last exposure (Day 21). To examine the immune response during chronic HP mice were intranasally inoculated with SR for 3 days per week for 25 weeks. All experiments included unexposed mice or saline exposed as negative controls.

\section{Cell Culture Medium}

RPMI (Cellgro, Mediatech Inc., Manassas, VA) containing 5\% heat-inactivated FCS (Hyclone; Rockford, IL), Glutamine (2mM), Penicillin/Streptomycin (100U/ml and $100 \mu \mathrm{g} / \mathrm{ml}$ ) (GIBCO cell culture Invitrogen; Carlsbad, CA) and 2nM 2-Mercaptoethanol (Fisher scientific; Pittsburgh, PA) was used in spleen and lung cell isolation experiments.

\section{Bronchoalveolar Lavage (BAL) and Lung Cell Isolation}

Mice were euthanized by $\mathrm{CO}_{2}$ asphyxiation and bronchoalveolar lavage (BAL) was performed by intratracheal injection of $1 \mathrm{ml}$ of PBS into the lung with immediate aspiration. The amount of fluid recovered was routinely $70 \%-80 \%$. To determine the extent of alveolitis in individual mice the BAL cells were recovered by centrifugation and live cells were counted using Trypan blue dye exclusion (Cellgro; Mediatech Inc., Manassas, VA). The BAL fluid was frozen at $-80^{\circ} \mathrm{C}$ until used in Enzyme Linked Immunosorbent Assay (ELISA) for cytokine and chemokine measurement.

To prepare single lung cell suspensions, lungs were perfused with sterile PBS to remove circulatory cells and lung lobes were removed. The lung lobes were minced into small pieces and digested with Collagenease $20 \mathrm{U} / \mathrm{ml}$ and Deoxyribonuclease I $40 \mu \mathrm{g} / \mathrm{ml}$ (Sigma-Aldrich, St. Louis, MO) for 45 minutes at $37^{\circ} \mathrm{C}$. The digested tissue was disrupted using a Stomacher ${ }^{\circledR} 80$ tissue processor (Brinkman Instruments, Westbury,

$\begin{array}{llll}\text { Day } & 1234567891011 & 12131415161718192021 \\ \text { SR exposure } & \uparrow \uparrow & \uparrow \uparrow \uparrow & \uparrow \uparrow \uparrow\end{array}$

Figure 2-1. Three week SR exposure. 
$\mathrm{NY}$ ) and debris removed by passage through a $70 \mu \mathrm{M}$ strainer (BD falcon). The cell suspension was layered on a discontinuous gradient consisting of $40 \%$ and $80 \%$ percoll (Sigma-Aldrich, St. Louis, MO). The cells were collected at the $40 \%$ - $80 \%$ percoll interface following centrifugation at $2000 \mathrm{rpm}$ for 20 minutes. The cells were washed with sterile PBS and live cells counted using Trypan Blue exclusion dye.

\section{RNA Extraction and Semi-Quantitative RT-PCR}

\section{RNA Extraction}

Total RNA was extracted from one lung lobe of individual mice using Trizol ${ }^{\circledR}$ (Invitrogen, Carlsbad, CA). Contaminating genomic DNA was removed by treating the RNA samples with DNase (Turbo DNA free, Ambion, Austin, TX). RNA concentration was determined by measuring the absorbance of UV light at 260nm, and 280nm using Ultraspec 2000 spectrophotometer (Pharmacia Biotech, Piscataway, NJ). The concentration of RNA $(\mu \mathrm{g} / \mathrm{ml})$ was calculated by multiplying the absorbance (A260), the dilution factor and $40 \mu \mathrm{g} / \mathrm{ml}$. To determine the quality of the RNA $1-2 \mu \mathrm{g}$ were loaded on a $4 \%$ agarose gel and analyzed for the presence of $18 \mathrm{~S}$ and $28 \mathrm{~S}$ ribosomal RNA subunits as an indicator of intact RNA.

\section{Semi-Quantitative RT-PCR}

Two $\mu$ g of RNA from individual samples was reverse transcribed into cDNA using a Promega Reverse Transcription System kit following manufacturer's instructions (Promega, Madison, WI). The cDNA synthesized from RNA was used as a template for end point PCR to be amplified using Taq Polymerase (Promega, Madison, WI). For semi-quantitave PCR, each PCR cycle was run with a denaturation step at $95^{\circ} \mathrm{C}$ for 30 seconds, followed by an annealing step at a primer-specific temperature (Table 2-1) for 30 seconds and an extension step at $72^{\circ} \mathrm{C}$ for 30 seconds. All the reactions were amplified for 35 cycles, except for $\beta$-actin, which was amplified for 28 cycles. After amplification $20 \mu l$ of product were loaded on a $1.8 \%$ agarose gel to be separated by agarose gel electrophoresis. The separated bands were visualized using ethidum bromide staining and were detected using an Alpha Image 2200 Multi-image light cabinet and alphaEase FC software (Alpha Innotech Corporation, San Leandro, CA).

\section{Flow Cytometry}

\section{Cell Surface Staining}

Phenotyping of BAL cells and lung cells was performed using flow cytometry. Cells $\left(2 \times 10^{5}\right.$ cells/tube) were incubated for 15 minutes on ice with $0.1 \mu \mathrm{g} / \mathrm{ml} \mathrm{Fc} \mathrm{block}$ (Anti-CD16/CD32 antibody) [BD Biosciences, San Diego, CA] to block Fc receptors. The cells were washed with $1 \mathrm{ml}$ FACS Buffer (PBS $+3 \%$ FCS) and centrifuged for 5 minutes at $1500 \mathrm{rpm}$. The cell pellet was resuspended in approximately $200 \mu \mathrm{FACS}$ 
Table 2-1. List of PCR primers used for PCR.

\begin{tabular}{|c|c|c|c|c|}
\hline $\begin{array}{l}\text { Primer } \\
\text { name }\end{array}$ & $\begin{array}{c}\text { Forward } \\
\text { primer }\end{array}$ & $\begin{array}{l}\text { Reverse } \\
\text { primer }\end{array}$ & $\begin{array}{l}\text { Product } \\
\text { length } \\
\text { (bp) }\end{array}$ & $\begin{array}{l}\text { Anneal. } \\
\text { temp. } \\
\left({ }^{\circ} \mathrm{C}\right)\end{array}$ \\
\hline IL-4 & $\begin{array}{c}\text { TCG GCA TTT TGA } \\
\text { ACG AGG TC }\end{array}$ & $\begin{array}{c}\text { GAA AAG CCC GAA } \\
\text { AGA GTC TC }\end{array}$ & 261 & 60 \\
\hline IL-6 & $\begin{array}{l}\text { GAC AAA GCC AGA } \\
\text { GTC CTT CAG AGA G }\end{array}$ & $\begin{array}{c}\text { CTA GGT TTG CCG } \\
\text { AGT AGA TCT C }\end{array}$ & 229 & 60 \\
\hline IL-17 & $\begin{array}{l}\text { CTC CAG AAG GCC } \\
\text { CTC AGA CTA C }\end{array}$ & $\begin{array}{l}\text { AGC TTT CCC TCC } \\
\text { GCA TTG ACA CAG }\end{array}$ & 142 & 60 \\
\hline IFN $\gamma$ & $\begin{array}{l}\text { TCA AGT GGC ATA } \\
\text { GAT GTG GAA GAA }\end{array}$ & $\begin{array}{c}\text { TGG CTC TGC AGG } \\
\text { ATT TTC ATG }\end{array}$ & 92 & 60 \\
\hline CXCL2 & $\begin{array}{l}\text { TGG GTG GGA TGT } \\
\text { AGC TAG TTC C }\end{array}$ & $\begin{array}{l}\text { GT TTG CCT TGA } \\
\text { CCC TGA AGC C }\end{array}$ & 285 & 65 \\
\hline T-bet & $\begin{array}{l}\text { GCC AGG GAA CCG } \\
\text { CTT ATA TG }\end{array}$ & $\begin{array}{l}\text { GAC GAT CAT CTG } \\
\text { GGT CAC ATT GT }\end{array}$ & 136 & 60.2 \\
\hline $\begin{array}{l}\text { Human } \\
\text { ROR } \gamma \mathrm{t}\end{array}$ & $\begin{array}{c}\text { CCG CTG AGA GGG } \\
\text { CTT CAC }\end{array}$ & $\begin{array}{l}\text { TGC AGG AGT AGG } \\
\text { CCA CAT TAC }\end{array}$ & 110 & 60 \\
\hline$\beta$-Actin & $\begin{array}{l}\text { GTG GGC CGC TCT } \\
\text { AGG CAC CA }\end{array}$ & $\begin{array}{c}\text { CGG TTG GCC TTA } \\
\text { GGG TTC AGG } \\
\text { GGGG }\end{array}$ & 245 & 60 \\
\hline
\end{tabular}


buffer and incubated with fluorochrome-conjugated antibodies specific for surface markers (Table 2-2) for $30 \mathrm{~min}$ on ice in the dark. The cells were washed twice with FACS Buffer and resuspended in a final volume of $200 \mu \mathrm{FACS}$ Buffer prior to analysis using a BD LSRII (BD, San Diego, CA) flow cytometer. Approximately, 30,000 to 50,000 events/tube were collected and the data was analyzed using FACSDiva Software (version 5.02).

Preparation of Bacterial Lysate for Intracellular Cytokine Staining

SR lysate was prepared by using Y-PER reagent (PIERCE, Rockford, IL). One gm of lyophilized SR was incubated with $7.5 \mathrm{ml}$ of Y-PER reagent for 20 minutes at room temperature with gentle rocking followed by three cycles of freeze and thaw 5 minutes each. The bacterial suspension was centrifuged at 8500rpm for 5 minutes at $4^{\circ} \mathrm{C}$ and the supernatant collected. The SR lysate was diluted in 10-20 volumes of PBS to remove SDS and concentrated using Y-10 concentrators (Millipore; Billerica, MA). The protein concentration in the SR lysate was measured using Bio-Rad protein assay following manufacturer's instructions (Bio-Rad Laboratories; Hercules, CA).

Intracellular Cytokine Staining

Intracellular cytokine staining was used to identify Th1 and Th17 cells. For Th1 or Th17 cell determination, lung cells were isolated from mice exposed to SR for three weeks. In a 96 well plate, at least $2 \times 10^{5}$ cells/well were plated and stimulated with media, SR lysate $(20 \mu \mathrm{g} / \mathrm{ml})$, or anti-CD3 antibody $(10 \mu \mathrm{g} / \mathrm{ml})$ for $7 \mathrm{hrs}$ at $37^{\circ} \mathrm{C}$. Golgi plug (BD Biosciences, San Diego, CA) was added for the final 4 hours of the culture to inhibit protein secretion. Cells were harvested and cell surface staining performed using antibodies to CD4, $\beta T C R$, and CD11b. Cells were fixed using $150 \mu 1$ BD Cytofix (BD Biosciences, San Diego, CA) for 15 minutes on ice and washed twice with permeabilization washing buffer ( $0.5 \%$ BSA $+0.5 \%$ Saponin in PBS). The cells were incubated with anti-IFN $\gamma$, anti-IL-17, or anti-IL-6 antibody for 30-45 minutes on ice, washed twice and analyzed on the BD LSRII.

\section{Histology}

The perfused left lung lobe was harvested and fixed overnight in neutral buffered formalin at $4^{\circ} \mathrm{C}$. The lung tissue was embedded in paraffin and sectioned into $8 \mu \mathrm{m}$ sections and stained with Hematoxylin and Eosin (H\&E) by the Department of Pathology, UTHSC.

\section{Statistical Analysis}

Values were expressed as means $\pm \mathrm{SD}$. For comparison of means between two populations of unpaired data, unpaired student's t-test was used. For comparison between more than two groups, One-way ANOVA followed by Tukey's post ANOVA test was 
Table 2-2. Characterization of innate and adaptive immune cell surface and intracellular markers.

\begin{tabular}{cc}
\hline Cell population & Cell markers \\
\hline Neutrophils & $\mathrm{CD} 45^{+}, \mathrm{CD} 11 \mathrm{~b}^{+}, \mathrm{Gr}-1^{\mathrm{hi}}$ or \\
Ly6G
\end{tabular}


used. GraphPad Prism 5 was used to perform all tests. Differences were considered significant at $\mathrm{P}<0.05$.

\section{RESULTS}

\section{Characterization of BAL Composition during Acute HP}

Measurement of alveolitis and characterization of BAL cellular composition are parameters to assess disease severity during HP. We analyzed the cell population in the airways by exposing C57BL/6J mice to SR. Following two daily exposures of SR (50 $\mu \mathrm{g}$ each), mice were sacrificed and BAL was performed. Cells were collected and counted for phenotypic characterization by flow cytometry. In exposed mice, we found a significant increase in alveolitis compared to unexposed control mice. Furthermore, there was an influx of neutrophils which represent approximately $80 \%$ of the total cells in the airways but there was no difference in the percentage of NK cells between SR exposed and unexposed mice (Table 2-3). In conclusion, acute HP is characterized by neutrophilic alveolitis.

\section{Expression of IL-17 and CXCL2 during Acute Phase of HP}

In the previous section, we demonstrated that acute HP was associated with neutrophilic alveolitis. Since IL-17 has been demonstrated to induce neutrophil

Table 2-3. C57BL/6J mice develop alveolitis and airway neutrophilia following acute exposure to SR compared to unexposed mice.

\begin{tabular}{|c|c|c|c|}
\hline Mice & $\operatorname{Alveolitis}^{\mathrm{a}}( \pm$ SD) & $\mathrm{PMN}^{\mathrm{b}} \%( \pm \mathrm{SD})$ & $\begin{array}{c}\text { NK cells }{ }^{\mathbf{b}} \% \\
( \pm \mathrm{SD})\end{array}$ \\
\hline $\begin{array}{l}\text { Unexposed } \\
\text { C57BL/6J }\end{array}$ & $0.19 \times 10^{6}( \pm 0.14)$ & $1.57( \pm 0.9)$ & $0.5( \pm 0.38)$ \\
\hline $\begin{array}{l}\text { Exposed } \\
\text { C57BL/6J }\end{array}$ & $2.15 \times 10^{6}( \pm 0.6) * *$ & $77.45( \pm 7.1)^{* * *}$ & $1.55( \pm 0.46)$ \\
\hline
\end{tabular}

C57BL/6J mice ( $\mathrm{n}=3-4 /$ group) were exposed to SR twice (50 $\mu \mathrm{g}$ each).

${ }^{a}$ BAL was performed and recovered cells were counted using Trypan Blue dye exclusion to exclude dead cells

${ }^{\mathbf{b}}$ Cells isolated from the BAL fluid were incubated with antibodies to various cell surface markers and analyzed by flow cytometry to determine cellular composition of the BALF. PMNs were identified as CD $11 \mathrm{~b}^{+} / \mathrm{Gr}-1^{\mathrm{hi}}$. NK cells were identified as NK $1.1^{+}$cells.

Results were expressed as means $\pm \mathrm{SD}$. Using unpaired student's t-test, the mean of the unexposed group was compared to the exposed group. ( $* * \mathrm{P}<0.005$ and $* * * \mathrm{P}<0.0001$ significant difference from the unexposed group.) 
chemokines e.g. CXCL1, CXCL2, and CXCL5 [161-163], we investigated whether there was an upregulation in IL-17 mRNA expression accompanied by upregulation in neutrophil chemokine (CXCL2) and neutrophilic alveolitis during acute HP. To fulfill this aim, mice were exposed twice daily to $50 \mu \mathrm{g}$ SR and lung IL-17 and CXCL2 mRNA expression were analyzed by semi-quantitative RT-PCR. RNA was isolated from lung homogenates of individual mice, reverse transcribed into cDNA and PCR was performed using primers specific for CXCL2 and IL-17. The results demonstrated that both IL-17 and CXCL2 mRNA expression increased following SR exposure as compared to unexposed mice (Fig. 2-2). These data demonstrated a correlation between neutrophil influx and IL-17 expression.

\section{Acute HP Is Associated with Th17 Differentiation and Effector Cytokines}

Several disease models associated with Th17 response were characterized by a neutrophil-rich environment $[169,170]$. During acute HP, we determined that HP is associated with IL-17 upregulation and neutrophilic alveolitis suggesting that HP is a Th17-mediated disease. Therefore, we examined the cytokines associated with Th17 response as well as its differentiation to determine whether they were present during the acute phase of HP. To fulfill this aim, C57BL/6J mice were exposed one time to $100 \mu \mathrm{g}$ SR and cytokine expression was analyzed by semi-quantitative RT-PCR. RNA was isolated from lung homogenates from individual mice 1, 3, 6, and 24 hours after SR exposure then reverse transcribed into cDNA to perform PCR using primers specific for IL-6, IL-21, IL-22 and IL-23. The housekeeping gene $\beta$-actin was used as an internal control. We found an upregulation in the proinflammatory cytokine IL-6 that is required for Th17 differentiation along with TGF $\beta$ (Fig. 2-3). IL-6 was upregulated at 1 hour post SR exposure and then it was downregulated at 24 hours. We found a slight upregulation in IL-22 which started following three hours of SR exposure. There was a weak expression in IL-21. Our data demonstrated weak expression of IL-23p19 which started at three hours and decreased by 24 hours. In conclusion, these results demonstrated the presence of cytokines associated with Th17 response and differentiation during acute HP.

\section{Characterization of BAL Composition during Granulomatous HP}

Continuous exposure to SR results in a disease shift towards the granulomatous stage. The hallmarks of the granulomatous phase of HP are granuloma formation in the peribronchiolar areas of the lungs and lymphocytic alveolitis. In contrast to acute HP, in granulomatous HP we found that neutrophils represented approximately $15 \%( \pm 1.32)$ of the total BAL composition; however, T cells represent $26 \%( \pm 6.2)$ of the total cells while $\mathrm{CD}^{+} \mathrm{T}$ cells represented $18 \%( \pm 4.9)$ of the total cells (Table 2-4). There was no difference in the percentage of NK cells. In conclusion, the results demonstrated a lymphocytic rather than neutrophilic alveolitis during granulomatous HP. 


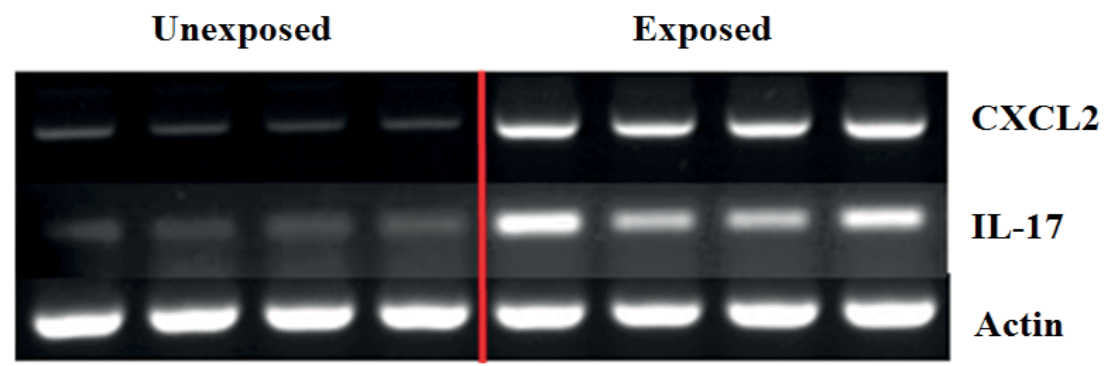

Figure 2-2. Expression of IL-17 and CXCL2 (MIP-2) during acute HP.

C57BL/6J mice (4/group) were exposed two times to SR (50 $\mathrm{gg})$. Total RNA was extracted from one lung lobe of individual unexposed and exposed mice then reverse transcribed into cDNA to use in RT- PCR using primers specific for IL-17 and CXCL2. The housekeeping gene $\beta$-actin was used as an internal control.

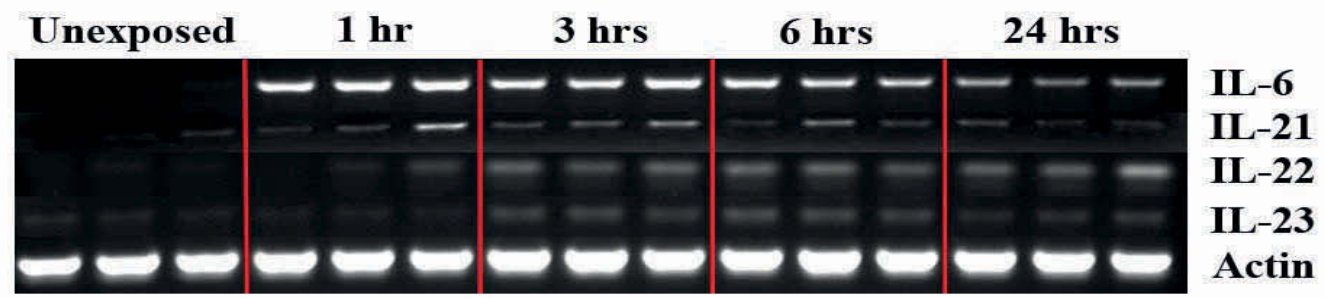

Figure 2-3. mRNA expression of Th17 cytokines during acute HP.

C57BL/6J mice ( $\mathrm{n}=3$ ) were exposed to $100 \mu \mathrm{g}$ SR then sacrificed after 1, 3, 6, or $24 \mathrm{hrs}$. Total RNA was extracted from one lung lobe of unexposed and exposed SR individual mice then reverse transcribed into cDNA to use in RT- PCR using primers specific for IL-6, IL-21, IL-22 and IL-23p19. The housekeeping gene $\beta$-actin was used as an internal control. 
Table 2-4. C57BL/6J mice develop lymphocytic alveolitis during granulomatous HP.

\begin{tabular}{|c|c|c|c|c|c|}
\hline Mice & $\begin{array}{l}\text { Alveolitis }^{\mathrm{a}} \\
\quad( \pm \text { SD })\end{array}$ & $\begin{array}{l}\text { PMN }^{\mathrm{b}} \\
\%( \pm \mathrm{SD})\end{array}$ & $\begin{array}{l}\text { NK cells }{ }^{b} \\
\%( \pm \text { SD })\end{array}$ & $\begin{array}{c}\text { T cells } \\
\%( \pm \text { SD })\end{array}$ & $\begin{array}{c}\mathrm{CD4}^{+} \\
\mathrm{T} \text { cells } \\
\%( \pm \mathrm{SD})\end{array}$ \\
\hline $\begin{array}{l}\text { Unexposed } \\
\text { C57BL/6J }\end{array}$ & $\begin{array}{l}0.3 \times 10^{6} \\
( \pm 0.13)\end{array}$ & $\begin{array}{c}1.66 \\
( \pm 0.71)\end{array}$ & $\begin{array}{c}15 \\
( \pm 0.38)\end{array}$ & $\begin{array}{c}4.8 \\
( \pm 0.92)\end{array}$ & $\begin{array}{c}4.03 \\
( \pm 0.7)\end{array}$ \\
\hline $\begin{array}{c}\text { SR } \\
\text { Exposed } \\
\text { C57BL/6J }\end{array}$ & $\begin{array}{c}5 \times 10^{6} \\
( \pm 1.6)^{* *}\end{array}$ & $\begin{array}{c}14.9 \\
( \pm 1.32)^{* * *}\end{array}$ & $\begin{array}{c}13.9 \\
( \pm 4.2)\end{array}$ & $\begin{array}{c}25.96 \\
( \pm 6.2)^{* *}\end{array}$ & $\begin{array}{c}18.4 \\
( \pm 4.9)^{* *}\end{array}$ \\
\hline
\end{tabular}

C57BL/6J mice ( $\mathrm{n}=3 /$ group) were exposed to SR ( $200 \mu \mathrm{g}$ each) three times a week for three weeks then sacrificed 4 days following last exposure.

${ }^{a} \mathrm{BAL}$ was performed and recovered cells were counted using Trypan Blue dye exclusion.

${ }^{\mathbf{b}}$ Cells isolated from the BAL fluid were incubated with antibodies to various cell surface markers and analyzed by flow cytometry to determine cellular composition of the BALF. PMNs were identified as CD $11 \mathrm{~b}^{+} / \mathrm{Gr}-1^{\text {hi }}$. NK cells were identified as NK1. $1^{+}$cells. T cells were identified as TCR $\beta^{+}$. CD4+ T cells were identified as TCR $\beta^{+} / \mathrm{CD} 4^{+}$. Results were expressed as means $\pm \mathrm{SD}$. Using unpaired student's t-test, the mean of the unexposed group was compared to the exposed group. $(* * \mathrm{P}<0.005$ and $* * * \mathrm{P}<0.0001$, significant difference from the unexposed group.) 


\section{Granuloma Formation during Granulamatous HP}

One of the hallmarks of HP is granuloma formation that is typically found in the peribronchiolar regions. To demonstrate that mice exposed to SR for three weeks develop this form of the disease, H\&E staining was performed on lung lobes isolated from exposed and unexposed mice (Fig. 2-4). The results demonstrated peribronchiolar granuloma in the lung interstitum. At this time point, if exposures were stopped, the granulomas resolved and the lung returned to normal (Fitzpatrick unpublished data).

\section{Granulomatous HP Is Associated with Activated CD4 ${ }^{+}$T Cells}

Granuloma formation in HP is T cell dependent; therefore, we examined the phenotype and activation state of the cells infiltrating the lung after 3 weeks of SR exposure. Following 3 weeks of SR exposure, unexposed or SR exposed mice were sacrificed at 4 days following last exposure. We isolated lung cells from mice exposed to SR for 3 weeks to determine the percentage and activation status of the T cells as well as innate cells. Single lung cell suspensions were prepared and incubated with fluorochrome conjugated antibodies to CD45, NK1.1, Gr-1, CD4, TCR $\beta$ chain, the early activation marker CD69 and CD25 (IL-2 $\alpha$ R, IL-2 high affinity receptor subunit).
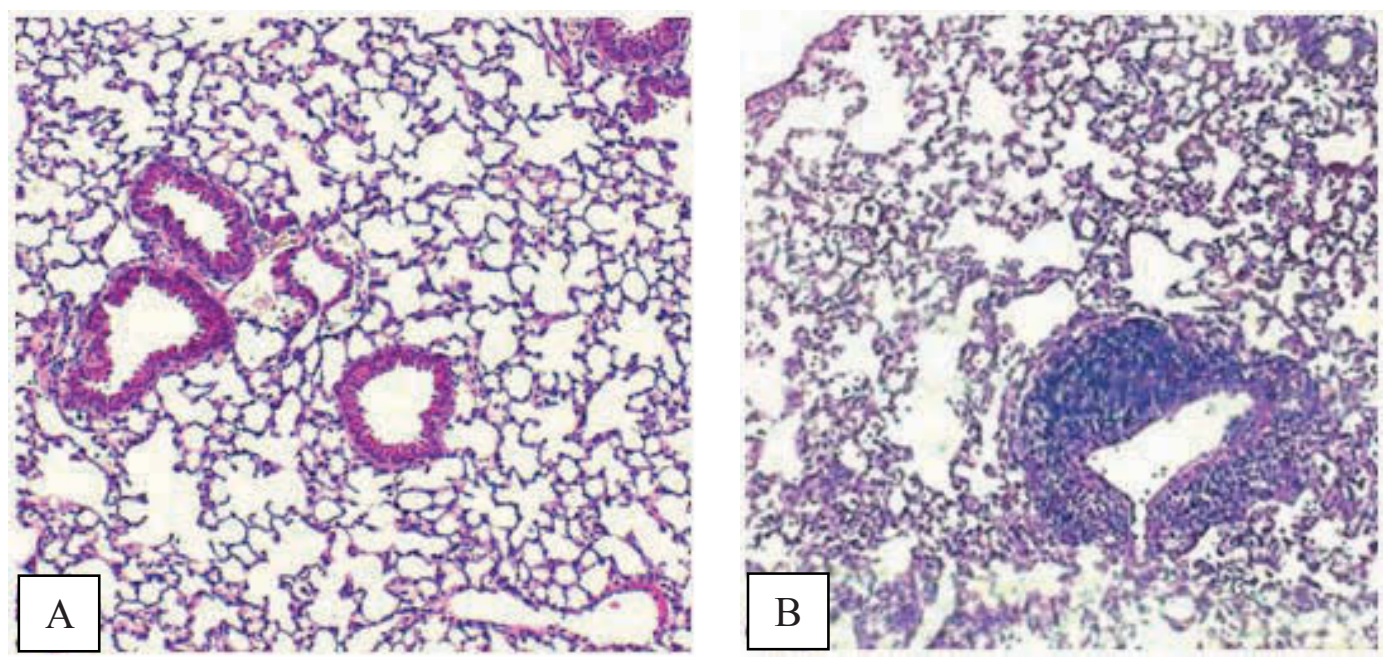

Figure 2-4. Peribronchiolar granuloma formation following 3 weeks of SR exposure.

Following 3 weeks of SR exposure, C57BL/6J mice were sacrificed and one lung lobe was used for H\&E staining. Staining lung sections from unexposed or SR exposed mice showed granuloma formation in the exposed mice (Original magnification 63X). Panel A represents lung section from unexposed mouse. Panel B represents lung section from SR exposed mouse. 
The results demonstrated an increase in the percentage of $\mathrm{T}$ cells $(16 \% \pm 3.6)$ in the lungs of exposed mice compared to unexposed mice $(13 \% \pm 1.8)$ (Fig. 2-5). Also, there was an increase in $\mathrm{CD} 4^{+} \mathrm{T}$ cells $(13 \% \pm 3.1)$ in the lungs of SR exposed mice compared to unexposed mice $(7.5 \% \pm 1.6)$. Furthermore, the $\mathrm{CD}^{+} \mathrm{T}$ cells from exposed mice were activated as measured by expression of the activation marker CD69. SR exposed mice showed a signficant increase in the percentage of $\mathrm{CD} 4{ }^{+} / \mathrm{CD} 69^{+} \mathrm{T}$ cells compared to unexposed mice $(6 \% \pm 2.2$ Vs. $0.7 \% \pm 0.2)$. However, we did not detect a signficant difference in CD25 (IL-2R) expression between unexposed and exposed groups. Interestingly, there was a signficant decrease in the percentage of NK cells in exposed mice compared to unexposed mice although this may be a dilutional effect. Finally, there was no difference in the percentage of PMNs between unexposed and SR exposed mice. In conclusion, granulomatous $\mathrm{HP}$ is associated with activated $\mathrm{CD}^{+} \mathrm{T}$ cells in the lungs.

\section{Granulomatous HP Is Associated with Th17 Immune Response}

In the previous section we found an influx of activated $\mathrm{CD} 4^{+} \mathrm{T}$ cells into the lungs; however, we do not know the type of these cells, i.e., Th1, Th2, or Th17. To determine the type of these cells, we used semi-quantitative RT-PCR to measure expression of T cell differentiation factors and cytokines. Following three weeks of SR exposure, total RNA was isolated from individual lung lobes of exposed mice as well as unexposed mice. Semi-quantitative RT-PCR was performed using primers for the Th1 cytokine IFN $\gamma$ and the Th1transcription factor T-bet, the Th2 cytokine IL-4, Th17 cytokine IL-17 and Th17 transcription factor ROR $\gamma$ t.

The results demonstrated a faint band for IL-4 in 2 / 3 exposed mice suggesting there was not much of a Th2 response developing at this time point. IFN $\gamma$ mRNA was increased in the exposed mice compared to the unexposed mice, demonstrating that this cytokine is associated with granulomatous inflammation; however, the Th1 transcription factor T-bet did not increase at the time points we examined. Since, both IL-17 and the Th17 transcription factor ROR $\gamma$ t expression were upregulated, these data suggest the development of Th17 response during this stage of the disease (Fig. 2-6).

The upregulation of IL-17 and ROR $\gamma \mathrm{t}$ mRNA in the lung homogenates suggested that Th17 cells had migrated into the lungs following SR exposure. In order to measure the frequency of Th1 and Th17 cells in the lungs, we performed intracellular cytokine staining. Mice were exposed to SR for 3 weeks and then lung cells were isolated and stimulated ex vivo with media or SR lysate for 6 hrs. Flow cytometry was performed using antibodies against CD45, CD4, IL-17 and IFN $\gamma$. We found an average of $16 \% \pm 1.7$ Th17 cells and $1.1 \% \pm 0.3 \mathrm{Th} 1$ cells in the lungs of SR exposed mice following ex vivo SR stimulation and an average of $0.36 \% \pm 0.15$ Th17 cells vs. $0.33 \% \pm 0.3$ Th1 cells in presence of media only. We also detected approximately $0.52 \% \pm 0.2 \mathrm{IFN} \gamma^{+} / \mathrm{IL}-17^{+} \mathrm{CD} 4^{+}$ T cells following SR stimulation (Fig. 2-7). In conclusion, granulomatous HP is associated predominately with a Th17 response and a low frequency of Th1 cells confirming the semi-quantitative RT-PCR results. 


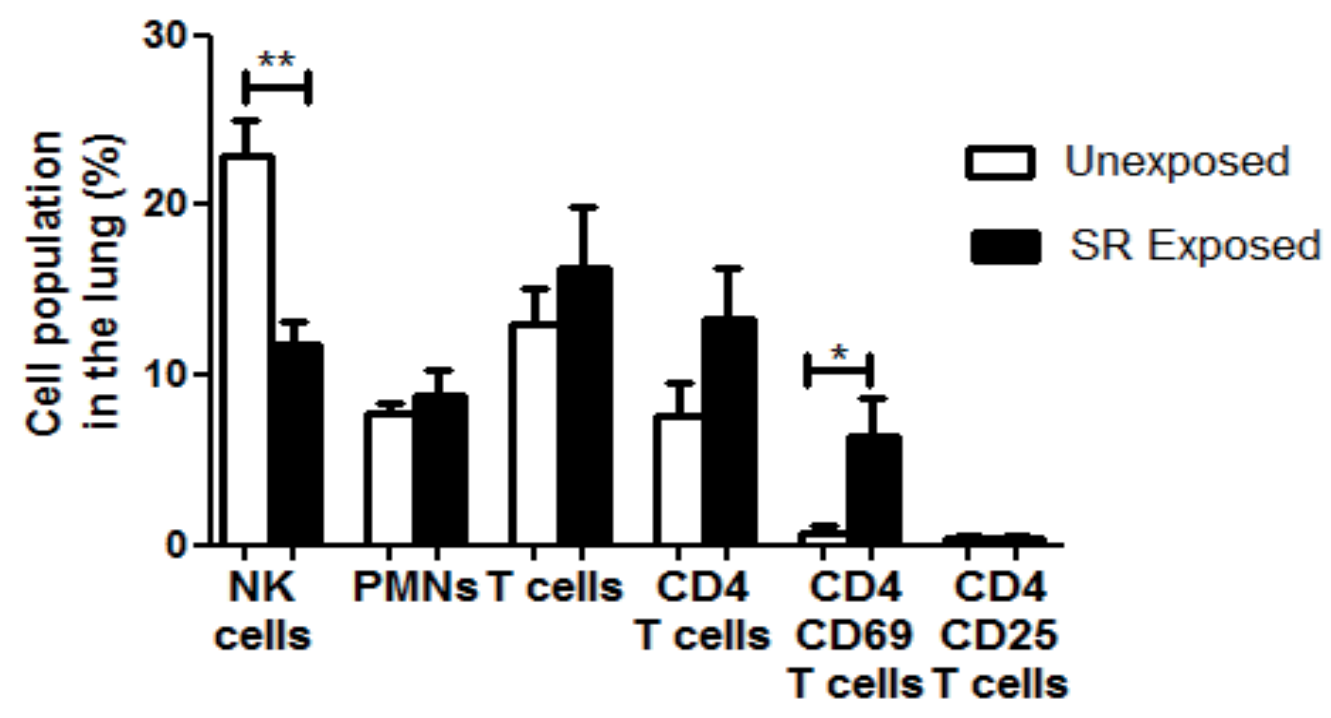

Figure 2-5. Cell surface staining showing the average percentage of different cell populations in the lung interstium of unexposed and SR exposed mice following 3 weeks of SR exposure.

Total immune lung cells were isolated from unexposed $(n=3)$ and exposed mice $(200 \mu \mathrm{g}$ SR for 3 weeks of exposure) then sacrificed 4 days later. Cells were stained for CD45, NK1.1, CD11b, TCR $\beta$, CD4, CD69, and CD25. Data was acquired on LSRII using FACS Diva Software. Approximately, 25,000 - 30,000 events were collected. Cells were gated as follows for NK cells CD $45^{+} / \mathrm{NK} 1.1^{+}$, PMNs CD $45^{+} / \mathrm{CD} 11 \mathrm{~b}^{+} / \mathrm{Gr}-1^{\text {hi }}$, T cells

$\mathrm{CD} 45^{+} / \mathrm{TCR} \beta^{+}, \mathrm{CD} 4^{+} \mathrm{T}$ cells $\mathrm{CD} 45^{+} / \mathrm{TCR} \beta^{+} / \mathrm{CD} 4^{+}$, activated $\mathrm{T}$ cells as $\mathrm{CD} 45^{+} / \mathrm{TCR} \beta^{+} / \mathrm{CD} 4^{+} / \mathrm{CD} 69^{+}$or $\mathrm{CD} 25^{+}$. Results were expressed as means \pm SD. Using unpaired student's t-test, mean of the unexposed group was compared to the exposed group. ( $* \mathrm{P}<0.02$ and $* * \mathrm{P}<0.002$, significant difference from unexposed group.) 


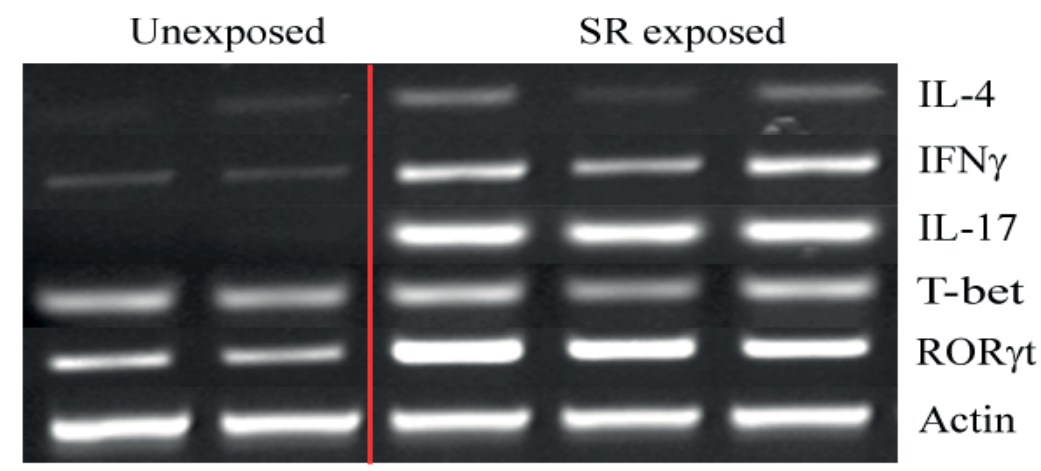

Figure 2-6. mRNA expression of different cytokines and $T$ cell transcription factors from unexposed and SR exposed mice during granulomatous HP.

C57BL/6J mice ( $\mathrm{n}=3$ ) were exposed to $200 \mu \mathrm{g}$ SR for three weeks then sacrificed 4 days following last exposure. Unexposed mice were used also as controls. Total RNA was extracted from one lung lobe of individual mice and reverse transcribed into cDNA to use in semi-quantitative RT- PCR using primers specific for IL-4, IFN $\gamma$, IL-17, ROR $\gamma$ t, and T-bet. The housekeeping gene $\beta$-actin was used as an internal control. 
(A)

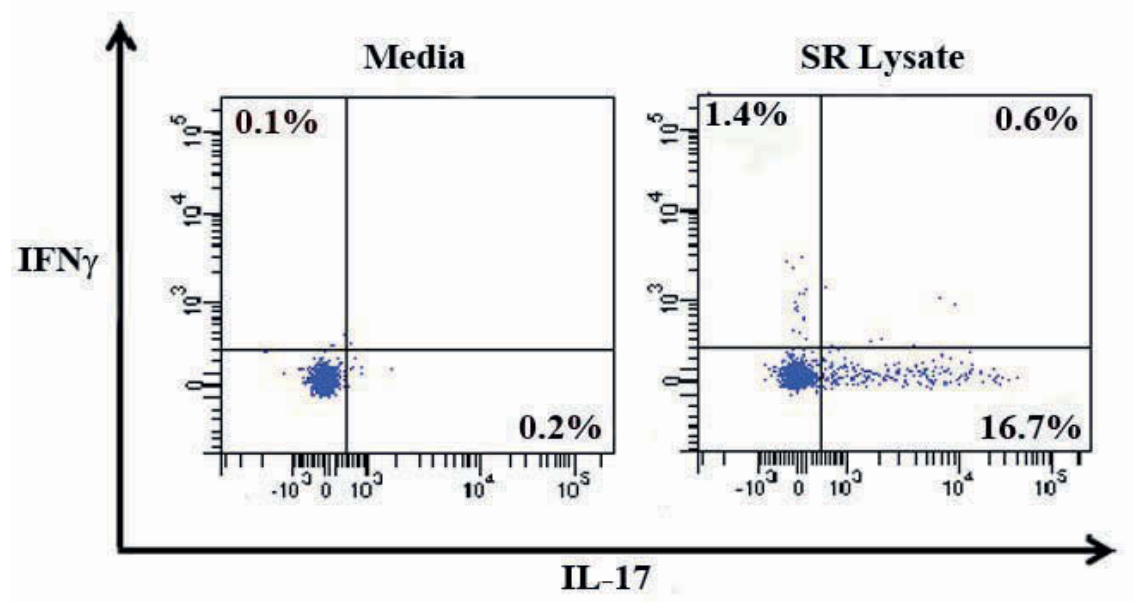

(B)

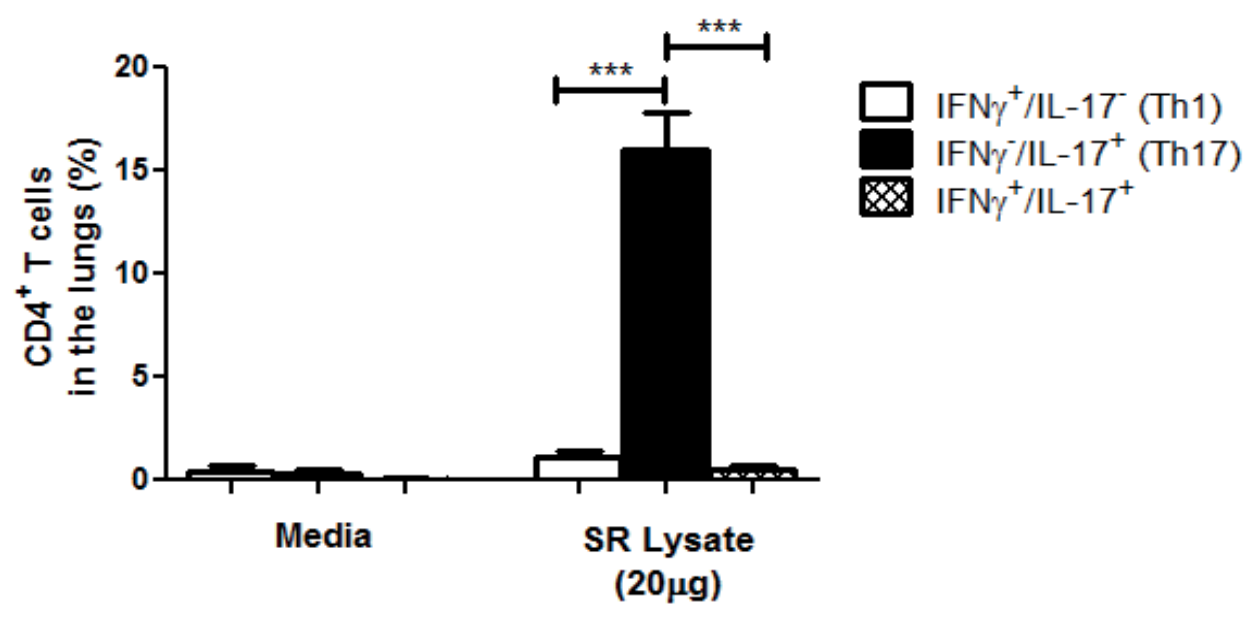

Figure 2-7. Intracellular cytokine staining showing percentage of Th1 and Th17 cells in the lungs following 3 weeks of SR exposed mice.

Mice ( $\mathrm{n}=5)$ exposed to $100 \mu \mathrm{g}$ SR for three weeks, then lung cells were isolated and stimulated in a 96 well plate $\left(5 \times 10^{5}\right.$ cells/well) ex vivo either with media or $20 \mu \mathrm{g}$ SR lysate for 3 hours followed by 3 hours of golgi plug at $37^{\circ} \mathrm{C}$ to accumulate cytokines inside the cell. Cells were stained for CD45, CD4, IL-17, and IFN $\gamma$. (A) Representative flow cytometry dot plot of IL-17 and IFN $\gamma$ expression by intracellular cytokine staining showed an increase in Th17 cells from the lungs of SR exposed mouse following SR ex vivo stimulation. (B) Average percentage of Th1, Th17, and IFN $\gamma^{+} / \mathrm{IL}-17^{+} \mathrm{CD} 4^{+} \mathrm{T}$ cells. Results were expressed as means $\pm \mathrm{SD}$. One way ANOVA followed by Tukey's post ANOVA test was used to compare means of all groups. $(* * * \mathrm{P}<0.0001$.) 


\section{Characterization of BALF during Chronic HP}

The previous results suggested that a predominant Th17 response was associated with granulomatous HP; however, this phase resolves and the lung returns to normal if the exposures are stopped (Fitzpatrick unpublished data). During the chronic form of HP, the granulomas become fibrotic and the disease does not resolve even if the exposures are stopped. This form of HP is associated with significant morbidity and mortality. To determine if the Th17 response is associated with this more severe phase or if the disease has switched to become more of a Th2 or Th1 response, we examined the T cell response following chronic exposure to SR. First, we characterized the compostion of BAL cells during the chronic phase of HP. Following 25 weeks of SR exposure, mice developed alveolitis which was mainly composed of neutrophils showing neutrophil influx during the chronic phase. Neutrophils represent $75 \%$ of the total cell in the airways compared to $1.25 \%$ in the unexposed mice. For T cells, there was an increase in the percentage of total $\mathrm{T}$ cells where $\mathrm{CD}^{+} \mathrm{T}$ cells constituted $50 \%$ of total $\mathrm{T}$ cells. Although, the percentage of NK cells in the BAL of unexposed mice is higher than exposed mice, their actual numbers are aproximately equal. Finally, there was not an increase in the percentage of CD8 T cells but the actual numbers of $\mathrm{CD} 8^{+} \mathrm{T}$ cells higher in the WT exposed mice compared to unexposed mice (Table 2-5).

\section{Chronic HP Is Associated with Granuloma Formation}

We analyzed granuloma formation during the chronic phase of the disease and found the granulomas were still located in the peribronchiolar region. As shown in Fig. 2-8, following 25 weeks of SR exposure, there was peribronchiolar granuloma formation as shown by H\&E staining.

\section{Chronic HP Is Associated with Activated CD4 ${ }^{+}$and $\mathrm{CD8}^{+} \mathrm{T}$ Cells}

To identify the immune cells infiltrating the lungs during chronic HP, we performed cell surface flow cytometry. Mice were exposed to SR for 25 weeks and the lung cells were isolated and incubated with fluorochrome conjugated antibodies to CD45, NK1.1, Gr-1, CD4, CD8, TCR $\beta$ chain, CD69 and CD25.

As observed in the granulomatous HP, the results demonstrated a significant decrease in the percentage of lung NK cells in the exposed mice compared to unexposed mice $(9.2 \% \pm 1.8$ Vs. $3.1 \% \pm 0.8)$. We also found a signficant increase in the percentage of neutrophils in SR exposed mice $(14.7 \% \pm 2.6)$ compared to unexposed mice $(6 \% \pm 1.4)$ suggesting a pathogenic role at that stage of the disease. Furthermore, T cells represent $(28 \% \pm 4.5)$ of the total immune cells in the lungs compared to $17 \% \pm 3.19$ in unexposed mice. $\mathrm{CD}^{+} \mathrm{T}$ cells represent $18 \% \pm 3.4$ of the total $\mathrm{T}$ cells in the lungs of mice following chronic SR exposure while $\mathrm{CD}^{+} \mathrm{T}$ cells represent $9 \% \pm 2.7$ of the total lung $\mathrm{T}$ cells of controls. The activation marker CD69. SR exposed mice showed a signficant increase in the percentage of $\mathrm{CD}^{+} / \mathrm{CD} 9^{+} \mathrm{T}$ cells compared to unexposed mice $(11 \% \pm 3 \mathrm{Vs} .2 .7 \%$ 
Table 2-5. Characterization of BAL cells during chronic HP.

\begin{tabular}{|c|c|c|c|c|c|c|}
\hline Mice & $\begin{array}{c}\text { Alveolitis }^{\mathrm{a}} \\
\quad( \pm \text { SD })\end{array}$ & $\begin{array}{l}\text { PMN }^{\mathbf{b}} \\
\%( \pm \text { SD })\end{array}$ & $\begin{array}{c}\text { NK } \\
\text { cells }{ }^{\mathrm{b}} \\
\%( \pm \mathrm{SD}) \\
\end{array}$ & $\begin{array}{l}\text { T cells }{ }^{b} \\
\%( \pm \text { SD })\end{array}$ & $\begin{array}{c}\text { CD4 T } \\
\text { cells }{ }^{b} \\
\%( \pm \text { SD }) \\
\end{array}$ & $\begin{array}{c}\text { CD8 T } \\
\text { cells }{ }^{b} \\
\%( \pm \text { SD }) \\
\end{array}$ \\
\hline $\begin{array}{l}\text { Unexposed } \\
\text { C57BL/6J }\end{array}$ & $\begin{array}{c}0.65 \times 10^{6} \\
( \pm 0.37)\end{array}$ & $\begin{array}{l}1.25 \\
( \pm 1)\end{array}$ & $\begin{array}{c}28.21 \\
( \pm 13.1)\end{array}$ & $\begin{array}{c}3.6 \\
( \pm 0.98)\end{array}$ & $\begin{array}{c}2.66 \\
( \pm 0.58)\end{array}$ & $\begin{array}{c}1.75 \\
( \pm 0.41)\end{array}$ \\
\hline $\begin{array}{c}\text { SR } \\
\text { Exposed } \\
\text { C57BL/6J }\end{array}$ & $\begin{array}{l}5.82 \times 10^{6} \\
( \pm 3.2)^{* * *}\end{array}$ & $\begin{array}{c}75.6 \\
( \pm 6.6)^{* * *}\end{array}$ & $\begin{array}{c}3.45 \\
(+/-0.73)\end{array}$ & $\begin{array}{c}8.4 \\
( \pm 3.39)^{* *}\end{array}$ & $\begin{array}{c}4.6 \\
( \pm 2.1)^{* *}\end{array}$ & $\begin{array}{c}2.52 \\
( \pm 1.1)\end{array}$ \\
\hline
\end{tabular}

C57BL/6J mice ( $\mathrm{n}=7-8 /$ group) were exposed to SR $(200 \mu \mathrm{g}$ each) three times a week for twenty five weeks then sacrificed 4 days following last exposure.

${ }^{\mathrm{a}} \mathrm{BAL}$ was performed and recovered cells were counted using Trypan Blue dye exclusion.

${ }^{\mathbf{b}}$ Cells isolated from the BAL fluid were incubated with antibodies to various cell surface markers and analyzed by flow cytometry to determine cellular composition of the BALF. PMNs were identified as $\mathrm{CD} 11 \mathrm{~b}^{+} / \mathrm{Gr}-1^{\text {hi }}$. NK cells were identified as NK1. $1^{+}$cells. T cells were identified as TCR $\beta^{+}, \mathrm{CD} 4 \mathrm{~T}$ cells as TCR $\beta^{+} / \mathrm{CD} 4+$, and CD8 T cells as $\mathrm{TCR} \beta^{+} / \mathrm{CD} 8^{+}$. Results were expressed as means $\pm \mathrm{SD}$. Unpaired student's t-test was used to compare the mean of both groups. ( $* * \mathrm{P}<0.005$ and $* * * \mathrm{P} \leq 0.0006$ significant difference from the unexposed group.) 

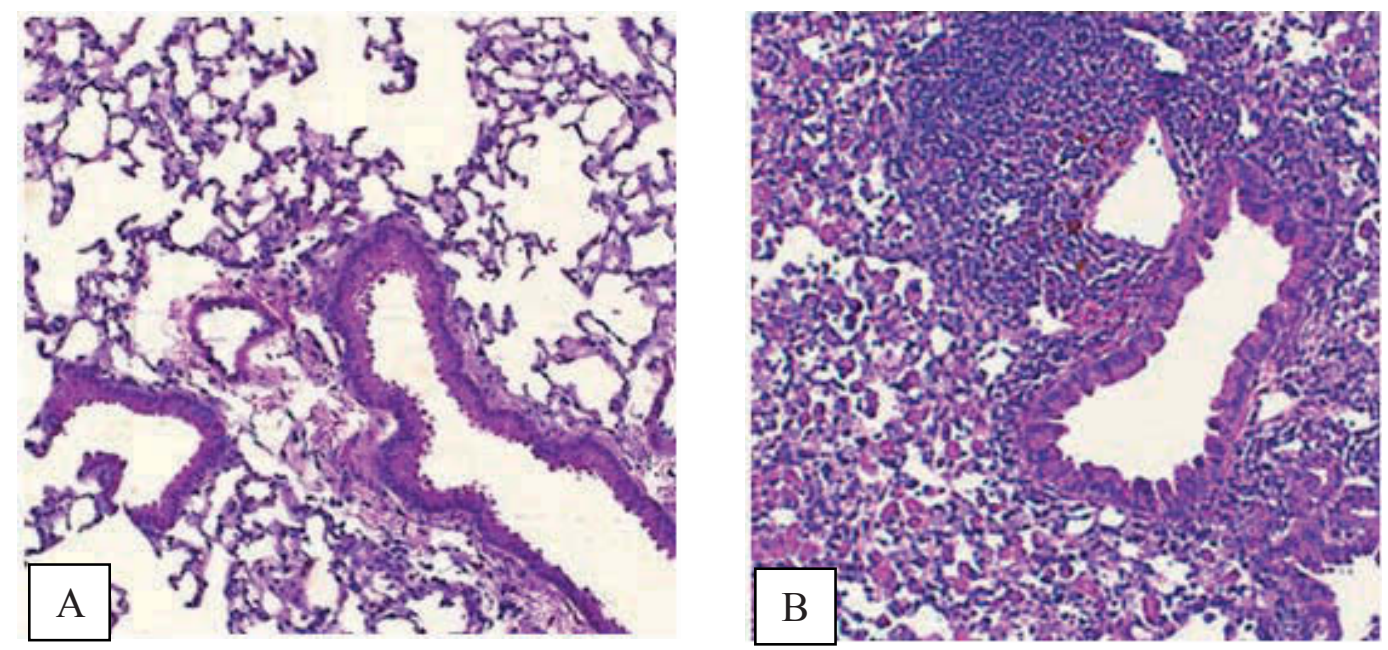

Figure 2-8. Peribronchiolar granuloma formation following 25 weeks of SR exposure.

Following 25 weeks of SR exposure, C57BL/6J mice were sacrificed and one lung lobe was used for H\&E. By staining lung sections from unexposed or SR exposed mice, we showed granuloma formation in the exposed mice (Original magnification 63X). Panel A represents lung section from unexposed mouse. Panel B represents lung section from SR exposed mouse following 25 weeks of SR exposure. 
$\pm 1.7)$. Also, there was a signficant increase in $\mathrm{CD}^{+} / \mathrm{CD} 25^{+} \mathrm{T}$ cells in SR exposed mice compared to unexposed mice $(3 \% \pm 0.8$ Vs. $1.25 \% \pm 0.6)$. Although there was not a significant diffenerce in the percentage of $\mathrm{CD} 8^{+} \mathrm{T}$ cells between unexposed and exposed mice, we found a signficant increase in the percentage of activated $\mathrm{CD} 8^{+} \mathrm{T}$ cells comapred to unexposed mice $(3.3 \% \pm 1.2$ Vs. $0.6 \% \pm 0.2)$ (Fig. 2-9). In conclusion, these results demonstrated that chronic HP is associated with neutrophils, activated $\mathrm{CD} 4^{+}$and $\mathrm{CD} 8^{+} \mathrm{T}$ cells in the lungs.

\section{Chronic HP Is Associated with a Th17 Immune Response}

Since the chronic phase of the disease is associated with fibrosis, it is important to identify the type of immune response that contributes to disease severity. We examined the $\mathrm{T}$ cell response following chronic exposure to SR to determine whether the influx of activated T cells into the lungs of exposed mice was composed of Th1, Th2, or Th17 cells. We used semi-quantitative RT-PCR to measure the expression of T cell differentiation factors and cytokines. Total RNA was isolated from the individual lung lobes of mice exposed to SR for 25 weeks. Semi-quantitative RT-PCR was performed using primers specific for Th1 cytokine IFN $\gamma$ and Th1 transcription factor T-bet, the Th2 cytokine IL-4, Th17 cytokine IL-17 and Th17 transcription factor ROR $\gamma$ t. The results demonstrated faint bands for IL-4 in 2/5 mice exposed to SR suggesting there was not a switch to a Th2 response at this time point. There was an increase in IFN $\gamma$ mRNA in the exposed mice compared to unexposed mice; however, mRNA for the Th1 transcription factor T-bet was not increased at this time point. Both IL-17 and the Th17 transcription factor ROR $\gamma t$ were increased suggesting that a Th17 response was maintained throughout this stage of the disease (Fig. 2-10).

The upregulation in IL-17 and ROR $\gamma \mathrm{t}$ mRNA levels in the lung homogenates suggested that Th17 cells had migrated into the lungs following SR exposure. In order to measure the level of Th1 and Th17 cells in the lungs, we performed intracellular cytokine staining. Mice were exposed to SR for 25 weeks and then lung cells were isolated followed by ex vivo overnight stimulation with media or SR in presence of activated adherent spleen cells. Flow cytometry was performed using antibodies against CD4, TCR $\beta$ chain (cell surface staining), IL-17 and IFN $\gamma$ (intracellular staining).

Following ex vivo stimulation with SR, or media, there was a significant increase in the percentage of Th17 cells compared to Th1 and IFN $\gamma^{+} / \mathrm{IL}-17^{+} \mathrm{CD}^{+} \mathrm{T}$ cells in the lungs of SR exposed mice (Fig. 2-11). In conclusion, these data indicated that a predominant Th17 immune response is associated with HP during its chronic stage suggesting a pathogenic role for Th17 response during HP. 


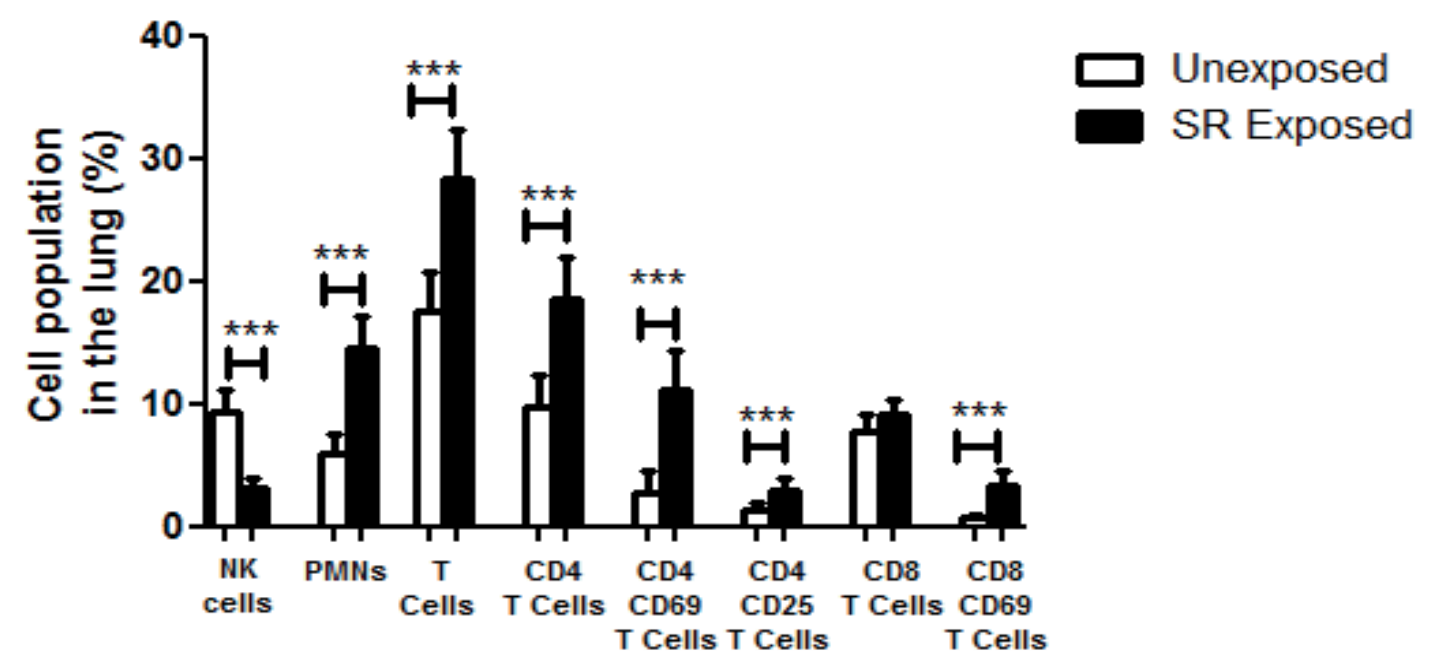

Figure 2-9. Cell surface staining showing the average percentage of different cell populations in the lung interstium of unexposed and SR exposed mice following 25 weeks of SR exposure.

Lung cells were isolated from unexposed mice $(n=7-8)$ and mice exposed to150 $\mu$ g SR for twenty five weeks. Cells were incubated with CD45, Gr-1, NK1.1, TCR $\beta$, CD4, CD8, CD69, and CD25. Data was acquired on LSRII using FACS Diva Software.

Approximately 20,000 events were collected. Cells were gated as follows for NK cells $\mathrm{CD} 45^{+} / \mathrm{NK} 1.1^{+}$, PMNs CD $45^{+} / \mathrm{CD}_{11} \mathrm{~b}^{+} / \mathrm{Gr}-1^{\mathrm{hi}}$, T cells $\mathrm{CD} 45^{+} / \mathrm{TCR} \beta^{+}$, CD 4 T cells $\mathrm{CD} 45^{+} / \mathrm{TCR}^{+} / \mathrm{CD} 4^{+}$, activated $\mathrm{T}$ cells as $\mathrm{CD} 45^{+} / \mathrm{TCR} \beta^{+} / \mathrm{CD} 4^{+}$or $\mathrm{CD} 8^{+} / \mathrm{CD} 69^{+}$or $\mathrm{CD} 25^{+}$. Results were expressed as means \pm SD. Unpaired student's t-test was used to compare mean of both groups. ( $* * * \mathrm{P}<0.001$, significant difference compared to unexposed group.) 


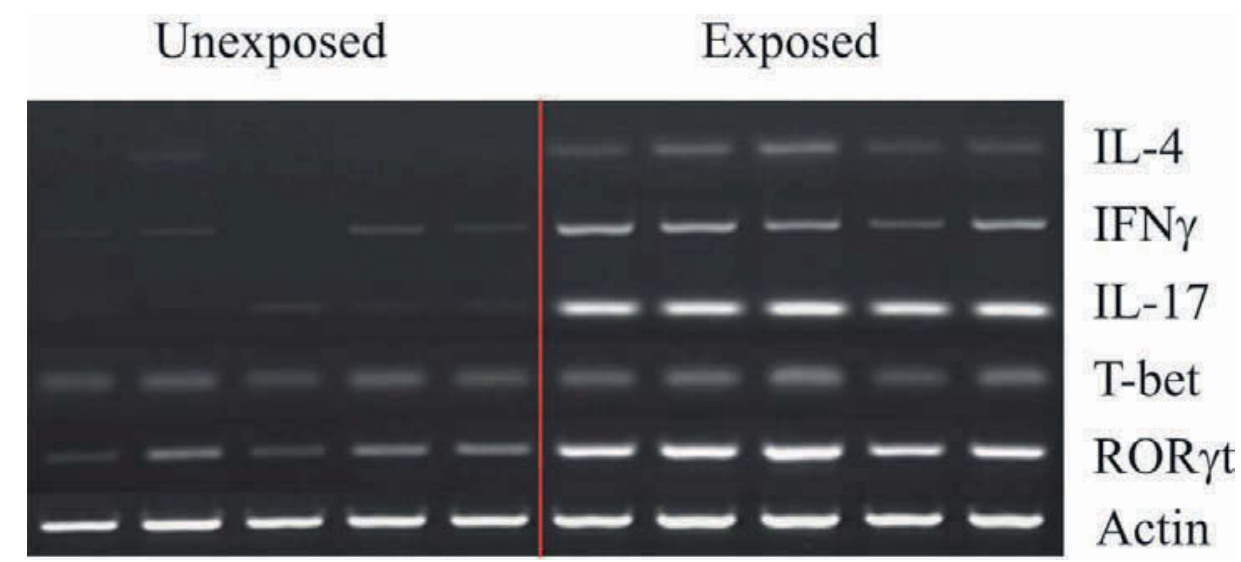

Figure 2-10. mRNA expression of different cytokines and $T$ cell transcription factors from unexposed and SR exposed mice during chronic HP.

C57BL/6J mice $(\mathrm{n}=5)$ were exposed to $150 \mu \mathrm{g}$ SR for 25 weeks. Unexposed mice were used as control. Total RNA was extracted from one lung lobe of individual mice and reverse transcribed into cDNA for RT- PCR using primers specific for IL-4, IFN $\gamma$, IL-17, ROR $\gamma$ t, and T-bet. The housekeeping gene $\beta$-actin was used as an internal control. 
(A)

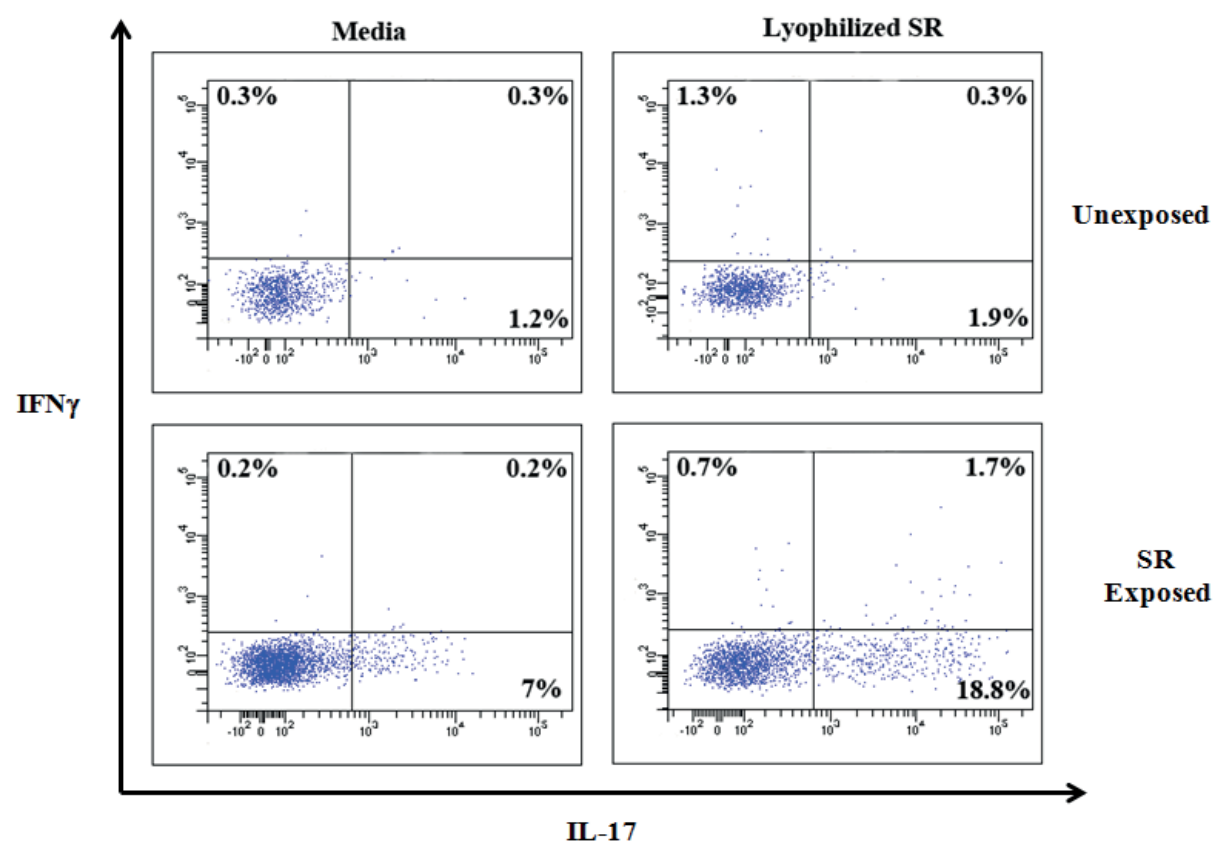

(B)

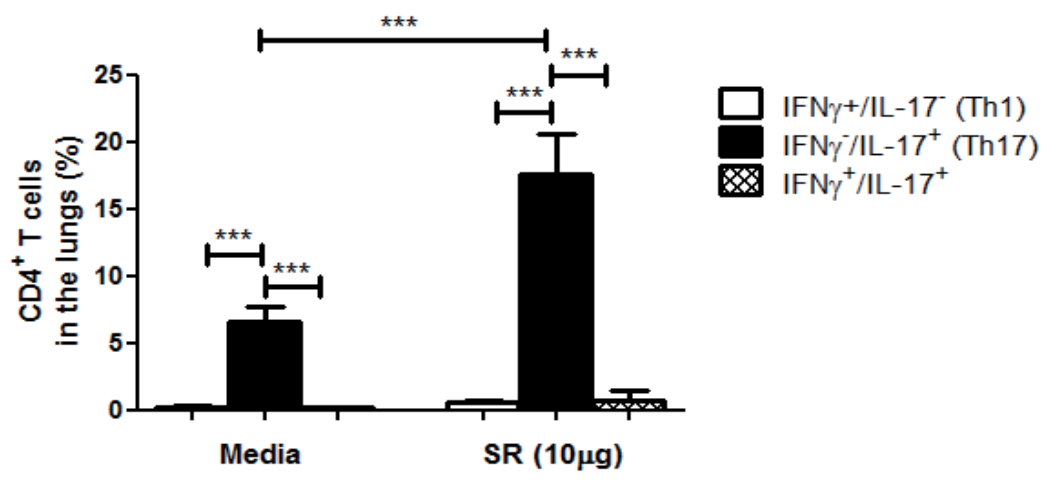

Figure 2-11. Intracellular cytokine staining showing percentage of Th17 and Th1 cells in the lungs following 25 weeks of SR exposure.

Lung cells were isolated from C57BL/6J unexposed mice ( $\mathrm{n}=3$ ) (used as a control) and mice exposed ( $\mathrm{n}=4$ ) to $150 \mu \mathrm{g}$ SR for 25 weeks. The cells were cocultured with C57BL/6J IFN $\gamma$ activated adherent spleen cells then stimulated ex vivo with media, or $10 \mu \mathrm{g}$ SR at $37^{\circ} \mathrm{C}$ for overnight incubation. Cells were stained for CD45, CD4, TCR $\beta$, IL-17, and IFN $\gamma$. (A) Representative Flow cytometry dot plot of IL-17 and IFN $\gamma$ expression from $\mathrm{CD}^{+} \mathrm{T}$ cells using intracellular cytokine staining showing increase in Th17 cells from the lungs of WT mouse following media, SR or PMA/Ionomycin ex vivo stimulation. (B) Average percentage of Th1, Th17, and IFN $\gamma^{+} / \mathrm{IL}-17^{+} \mathrm{CD} 4^{+} \mathrm{T}$ cells in the lungs of SR exposed mice. Results were expressed as means \pm SD. One way ANOVA followed by Tukey's post ANOVA test was used to compare means of all groups $(* * * \mathrm{P}<0.0001)$. 


\section{DISCUSSION}

\section{Acute HP: Neutrophilic Alveolitis and IL-17 Expression}

The goal of this chapter was to determine the T cell response during HP. We hypothesized that HP is associated with the Th17 cell response based on the correlation between IL-17 upregulation in the lungs during HP and the neutrophil-rich environment which is one of the hallmarks of Th17 response. Our initial studies demonstrated a neutrophilic alveolitis during acute HP accompanied by an upregulation in mRNA expression of CXCL2 and IL-17. This experiment was only showing the correlation between expression of IL-17 and CXCL2 and neutrophil recruitment; however, the direct effect of IL-17 on neutrophil recruitment during acute HP was not tested. Previous data in our lab have showed that neutralization of IL-17 over a period of 3 weeks of SR exposure decreased significantly the influx of neutrophils into the airways compared to control mice. In summary, the data demonstrated the relation between IL-17 and CXCL2 expression and neutrophilic alveolitis during acute HP.

The previous data indicated that the initial response during acute HP is inclined towards a Th17 cell response later on during adaptive phase of HP. However, further experiments are required to prove this statement. Therefore, we measured the cytokines associated with a Th17 immune response as well as cytokines required for Th17 cell differentiation during acute HP. Using semi-quantitative RT-PCR, we identified an upregulation in IL-6 while IL-21, IL-22, and IL-23 were detected but weakly expressed. Since IL-6 is required for Th17 differentiation; therefore, the upregulation in IL-6 might indicates a progression to Th17 cell development later on in the disease. The expression of IL-23 at that stage of HP might be required for induction of IL-17 from other innate cells e.g. $\gamma \delta$ T cells and NKT cells [160]. As mentioned in the introduction, IL-22 plays a protective role at mucosal surfaces against bacterial infections through induction of several mechanisms e.g. induction of antimicrobial peptides, enhancement of barrier integrity. Although, it was reported that IL-22 plays a protective role against development of fibrosis during HP, its role during acute HP was not addressed. In conclusion, these results mean a probable Th17 response later on during adaptive stages of HP (granulomatous and chronic HP).

\section{Granulomatous HP and the Th17 Response}

The detection of IL-6, IL-21, IL-22, and IL-23 during acute HP suggested that a Th17- associated micro-environment developed after exposure to SR. Therefore, we wanted to determine whether a Th17 immune response was present during the granulomatous phase of the disease and so we examined mice that had been exposed to SR for 3 weeks. The results demonstrated a lymphocytic alveolitis during granulomatous HP rather than neutrophilic, where $\mathrm{CD} 4^{+} \mathrm{T}$ cells constituted a large percentage of $\mathrm{T}$ cells. As mentioned earlier in the introduction, $\mathrm{CD}^{+} \mathrm{T}$ cells play a pathogenic role in HP. Also, they are required for granuloma formation. Therefore, the presence of $\mathrm{CD}^{+} \mathrm{T}^{\mathrm{T}}$ cells 
in the airways indicated their pathogenic role in the lungs. Since H\&E staining showed that granulomatous HP was associated with peribronchiolar granuloma, we measured the phenotype of the T cells in the lung. As expected we found an increase in the percentage of total T cells and the $\mathrm{CD} 4^{+} \mathrm{T}$ cells were activated as compared to unexposed mice although the difference did not reach the level of statistical significance. By using only 3 mice per group, the statistical analysis was underpowered. The presence of activated $\mathrm{CD}^{+} \mathrm{T}$ cells in the lungs suggested that the $\mathrm{T}$ cells encountered SR antigen presented by APCs in the lungs. Also, it indicated that $\mathrm{T}$ cells are secreting their effector cytokines and their interaction with APCs activates APCs to secret cytokines that contribute to lung pathology. Also, there was no difference in the percentage of PMNs isolated from the lung between unexposed and SR exposed mice. Since the mice were sacrificed 4 days after last exposure, that might explain why we found low percentage of PMNs in the lungs. The PMNs were recruited shortly after SR exposure then they died later on, while the $T$ cells remained activated because they encountered SR antigen in the lungs presented by APCs. Finally, there was a signficant decrease in the percentage of NK cells in the lungs of exposed mice compared to unexposed mice. NK cells could be outnumbered by recruitment of other cell populations in the lungs. In conclusion, these results suggested that during granulomatous HP, granuloma formation is associated with activated $\mathrm{CD} 4^{+} \mathrm{T}$ cells.

Next, we wanted to examine the type of the $\mathrm{CD} 4^{+} \mathrm{T}$ cells in the lungs, whether Th1, Th2, or Th17 developed during granulomatous HP. Using semi-quantitative RT-PCR, we found an upregulation in the Th17 transcription factor, ROR $\gamma \mathrm{t}$ and Th17 signature cytokine, IL-17 suggesting that Th17 cells were present in the lungs and the presence of IL-17 suggested that they are actively secreting IL-17. There was an increase in the Th1 cytokine IFN $\gamma$ but no increase in the transcription factor T-bet which indicated that the increase in IFN $\gamma$ was not from Th1 cells, but it could be from another type of cell. Previous results from our lab showed that neutrophils are an important source of IFN $\gamma$ during HP [129]. Finally, we did not detect a significant upregulation of IL-4 in the exposed mice as compared to the unexposed mice demonstrating that a Th2 response had not developed. The absence of Th2 response during HP indicated that induction of fibrosis during chronic stage is attributed to a different $\mathrm{T}$ cell response other than Th2 response; however, we still need to examine the $\mathrm{T}$ cell response during the chronic stage to reach to this conclusion. Since the cytokine profile present in the lung suggested both Th17 cells as well as Th1 cells (IFN $\gamma$ production), we measured the level of SR antigen specific Th1 and Th17 cells in the lungs using intracellular cytokine staining. We found that SR antigen specific Th17 cells were the dominant effector T cells in the lungs after 3 weeks of SR exposure while SR antigen specific Th1 represented a small percentage which is confirmed by PCR results. The presence of Th17 cell response in the lungs associated with granuloma formation suggested to us that these cells could play a role in the pathogenesis of HP during this stage. One study demonstrated the association of Th17 response with granuloma formation. Mouse infection with schistosome egg antigen with Complete Freund's Adjuvant (SEA/CFA) resulted in granuloma formation accompanied by Th17 response. In conclusion, Th17 cells were associated with granuloma formation during granulomatous HP suggesting their pathogenic role during HP. 


\section{Chronic HP and Th17 Response}

The next stage of our studies was to determine if the Th17 cell response was associated with the chronic phase of the disease or if the response switched to a Th1 or Th2 response. This is important since the chronic response is associated with fibrosis and significant morbidity and mortality. We exposed mice to SR for 25 weeks and measured several parameters of disease severity. The results demonstrated a neutrophilic alveolitis with an increase in the number of T cells specifically $\mathrm{CD} 4^{+} \mathrm{T}$ cells, in exposed mice compared to unexposed mice. These results were not surprising since neutrophils produce mediators such as collagenases and gelatinases that cause tissue remodeling and fibrosis which is the hallmark of chronic HP [113]. The increase in $\mathrm{CD}^{+} \mathrm{T}$ cells which reflected to us their presence in the lung was also expected, since we found chronic HP was associated also with granuloma as in the granulomatous phase using H\&E staining. To identify the cellular composition of the interstitial lung cell population during chronic HP, we harvested the lungs after 25 week of chronic exposure protocol and performed flow cytometry. We identified an increase in the percentage of lung neutrophils following SR exposure compared to unexposed mice. In chronic HP patients, neutrophils express gelatinases and collagenases which might contribute to fibrotic response during chronic HP [113]. We also found that $\mathrm{T}$ cells represent the largest population in the lungs of exposed mice especially activated $\mathrm{CD} 4^{+} \mathrm{T}$ cells and $\mathrm{CD} 8^{+} \mathrm{T}$ cells. The presence of activated $\mathrm{CD} 4{ }^{+} \mathrm{T}$ cells suggested that $\mathrm{T}$ ells encountered SR antigen in the lungs presented by APCs and the activated $\mathrm{CD}^{+} \mathrm{T}$ cells are producing effector cytokines. Although $\mathrm{CD}^{+} \mathrm{T}$ cells are the pathogenic T cells in HP, we had found activated CD $8^{+}$ $\mathrm{T}$ cells during chronic HP. Previous studies in our lab demonstrated that $\mathrm{CD}^{+} \mathrm{T}$ cells do not produce IL-17. These results suggested that during chronic HP, granuloma formation is associated with activated $\mathrm{CD} 4^{+}, \mathrm{CD} 8^{+} \mathrm{T}$ cells, and neutrophils.

To determine the type of activated $\mathrm{CD}^{+}{ }^{\mathrm{T}}$ cell whether Th1, Th2, or Th17; we used semi-quantitative RT-PCR. Following 25 weeks of SR exposure, we found an upregulation in the Th17 transcription factor (ROR $\gamma \mathrm{t}$ ), IL-17 and IFN $\gamma$ without change in T-bet and IL-4 expression in SR exposed mice suggesting presence of both Th1 and Th17 cells and the absence of Th2 cells at that stage. The absence of Th2 cells indicated that the fibrosis process in that model is mediated by another type of T cells presumably by Th17 cells. Since the cytokine profile present in the lung suggested that both Th17 cells as well as Th1 cells (IFN $\gamma$ production) were present, we measured the level of SR antigen specific Th1 and Th17 cells in the lungs using intracellular cytokine staining. The results demonstrated that SR antigen specific Th17 cells are still the dominant effector $\mathrm{T}$ cells in the lungs after 25 weeks of SR exposure while SR antigen specific Th1 represented a small percentage. Th17 cells could be contributing to the fibrosis process during chronic HP through production of IL-17 as an effector cytokine. IL-17 consequently induces neutrophil recruitment into the lungs which is already observed during chronic HP. Finally, neutrophils might play a role in fibrosis through secretion of gelatinases and collagenases that cause tissue remodeling and eventually fibrosis [113]. In conclusion, Th17 cells might play an indirect role in fibrotic response possibly through neutrophil recruitment during chronic HP. 
IFN $\gamma^{+} / \mathrm{IL}-17^{+} \mathrm{CD} 4^{+} \mathrm{T}$ cells were only observed following PMA/Ionomycin and they were absent following ex vivo media and SR stimulation. Th1/Th17 cells can be generated from Th17 cells following IL-12/IFN $\gamma$ stimulation [171], hence, PMA/Ionomycin might be stimulating IL-12 and IFN $\gamma$ production that can switch Th17 response to Th1/Th17 response. The origin of the double producers is not clear but there have been several studies addressing this issue. Hirota et al. demonstrated that the double producers originally derived from Th17 cell [172]. Since these cells were enriched in target organs of several autoimmune diseases, it was suggested that these cells were pathogenic; whether these cells were pathogenic or protective it was not shown clearly in the literature [173].

\section{Th17 Response and HP: A Correlation Not a Cause and Effect Study}

Our results from this chapter demonstrated a correlation between disease severity and Th17 response. The role of Th17 cells in HP still remains to be answered. Simonian et al. demonstrated that reconstitution of TCR $\beta / \delta \mathrm{DKO}$ mice (mice lacking $\mathrm{CD} 4^{+}, \mathrm{CD} 8^{+}$, and $\gamma \delta \mathrm{T}$ cells) with splenic naïve $\mathrm{CD} 4^{+} \mathrm{T}$ cells followed by SR exposure for 4 weeks resulted in lung inflammation and increase in lung collagen content as a measurement of fibrosis. This coincided with an increase in IL-17 expression in the lung homogenates compared to those mice exposed to PBS. These data demonstrated the importance of $\mathrm{CD} 4^{+} \mathrm{T}$ cells as inducers of lung inflammation and fibrosis during HP in a direct or indirect way. Furthermore, repeated exposure of WT mice to SR for 4 weeks increases the frequency of Th17 in the lungs [62]. To identify the role of IL-17 in HP Simonian et al. and Joshi et al used IL-17RKO and IL-17KO mice and exposed them to SR [62, 174]. Both studies demonstrated a significant reduction in lung inflammation $[62,174]$ and collagen deposition in IL-17R knockout exposed mice compared to WT exposed mice [62]. Even though IL-17 is one of the signature cytokines of Th17, it is still produced by other immune cells [157-159]. Adoptive transfer experiment could be performed to investigate the direct role of Th17 during HP. In this experiment, Th17 cells from SR exposed mice will be adoptively transfered into TCR $\beta / \delta$ DKO mice followed by SR exposure then lung inflammation and collagen content will be tested. In summary, the authors concluded from their data that Th17 plays a pathogenic role of Th17 during HP [62]; yet, how these cells cause lung fibrosis remains to be an open question.

Our results demonstrated an increase in IL-22 in the lungs following SR exposure. The role of IL-22 in lung fibrosis was previously addressed during HP by Simonian et al. [175]. In a HP mouse model caused by $B$. subtilis, IL-22 is produced mainly by a subset of $\gamma \delta$ T cells. IL-22 expression from $\gamma \delta$ T cells requires stimulation of the Aryl hydrocarbon receptor (AhR) by environmental toxins [176]. In B. subtilis mouse HP model, AhR mutant mice $\left(\mathrm{AhR}^{d / d}\right)$ are characterized by reduced IL-22 expression by $\gamma \delta \mathrm{T}$ cells along with increase in collagen deposition [175]. To confirm these data, recombinant IL-22 was injected into $\left(\mathrm{AhR}^{d / d}\right)$ mice and TCR $\delta \mathrm{KO}$ mice exposed to $B$. subtilis causing reduction in collagen content as well as reduced lung $\mathrm{CD}^{+}{ }^{+} \mathrm{T}$ cell recruitment and lung inflammation. In the same line of evidence, neutralization of IL-22 in WT mice exposed to $B$. subtilis for 4 weeks resulted in an increase in the amount of 
collagen and the number of $\mathrm{CD}^{+} \mathrm{T}$ cells in the lungs compared to mice treated with isotype antibody [175]. Taken together, these data suggest that IL-22 plays a protective role in the $B$. subtilis induced HP model. In Farmer's Lung disease model, the frequency of $\mathrm{CD}^{+} \mathrm{T}$ cells expressing IL-22 in lungs is low [62] and IL-22 produced by $\gamma \delta \mathrm{T}$ cells during $B$. subtilis induced HP is protective [175]. Although both models are induced by different agents, a correlation can be drawn from these two studies. The low expression of IL-22 in Th17 cells during HP might be contributing to the disease severity. In conclusion, we hypothesized that HP is a Th1/Th17 mediated disease, yet the contribution of each subset to disease severity is still enigmatic. Several experimental disease models showed the coexistence of both Th1 and Th17 cells during the disease progression. In the $T$. cruzi mouse infection model, a Th1 response develops upon antigen challenge as well as a strong Th17 response [170]. In experimental autoimmune uveitis as a model for human uveitis, either Th1 or Th17 cells can cause the disease [177]. Furthermore, in experimental autoimmune encephalomyelitis (EAE), both Th1 and Th17 cells infiltrate the CNS [178] and antigen specific Th1 or Th17 cells generated in vitro adoptively transfer the disease into naïve mice [179]. Thus, all the previous studies showed the coexistence of Th1 and Th17 in different diseases. The cooperation between both types of T cells was demonstrated in EAE model when Th1cells collaborate with Th17 cells by facilitating the entry of Th17 into CNS [180]. In summary, our results from this chapter demonstrated a predominant Th17 response with low percentage of Th1 cells during granulomatous and chronic HP. 


\section{CHAPTER 3. T-BET IN HP*}

The results from chapter 2 indicated that both granulomatous and chronic HP are associated with a predominant Th17 response. However, HP was considered as Th1-mediated disease, since IFN $\gamma$ was required for granuloma formation during HP. In order to clarify the role of IFN $\gamma$ in the disease, we examined the regulation more closely. In this chapter, we will identify the cytokines required for IFN $\gamma$ production from neutrophils, determine role of T-bet in the regulation of Th17 development and disease severity. The results demonstrated that combination of IL-12/ IL-18 or IL-15/IL-18 were required for induction of IFN $\gamma$ from neutrophils and T-bet inhibits Th17 response and controls disease severity during HP.

\section{INTRODUCTION}

T-bet (T-box expressed in $\mathbf{T}$-cells) is the master transcription factor required for Th1 differentiation [8]. Although the name suggests that it is expressed by T cells only, other immune cells such as dendritic cells [9], NK, and NKT cells [10] can express it as well. During Th1 polarization, the expression of T-bet in naïve T cells is induced by the synergistic effect of TCR stimulation and IFN $\gamma-$ STAT1 signaling. Induction of T-bet induces expression of the IL-12R $\beta 2$ subunit on the cell surface increasing responsiveness of the cell to IL-12. With additional IL-12 stimulation more T-bet is induced resulting in amplification of the reaction. T-bet also induces an array of genes such as IFN $\gamma, \mathrm{CCL} 4$, CCL3 and CXCR3 (chemokine receptor for CCL3 and CCL4). Since these chemokines are important for Th1 cell migration T-bet indirectly contributes to Th1 cell recruitment to sites of inflammation [181]. Although, IFN $\gamma$ is the prototypic cytokine produced by Th1 cells, other immune cells, such as $\mathrm{CD}^{+} \mathrm{T}$ cells, NK cells, NKT cells, antigen presenting cells and neutrophils [182-184] can express it as well. Neutrophils are considered as an important source of IFN $\gamma$ during HP [129]; however, it is not known how IFN $\gamma$ expression is induced in neutrophils and whether T-bet is expressed by neutrophils is still unclear. Several cytokines have been reported to stimulate IFN $\gamma$ from neutrophils including IL-12 alone or in combination with IL-2, IL-15, IL-18, or LPS. The combination of IL-12 with IL-15 was the most efficient at inducing IFN $\gamma$ production from human neutrophils [183]. In a different study neutrophils isolated from human uterine endometrium tissue were able to produce IFN $\gamma$ upon stimulation with a combination of TNF $\alpha$ and IL-12 [182]. Finally, peritoneal murine neutrophils produced IFN $\gamma$ following stimulation with LPS [185]. In this chapter we will identify the cytokines that induce IFN $\gamma$ production from neutrophils isolated from SR exposed mice.

Regulation of IFN $\gamma$ production and Th1 differentiation is not the lone function of T-bet. T-bet can also inhibit Th2 differentiation by binding to GATA-3 (a Th2 transcription factor) and hence sequester GATA-3 from binding to IL-5 and IL-13

*(Adapted with permission from Abdelsamed, H.A., et al., T-bet controls severity of hypersensitivity pneumonitis. J Inflamm (Lond), 2011. 8(1): p. 15 [186].) 
promoters. T-bet also binds to Runx-3 transcription factor and both bind to the IL-4 silencer to inhibit IL-4 expression [181]. Recently, Lazarevic et al. showed that T-bet binds to Runx-1 transcription factor inhibiting the expression of Th17 specific transcription factor ROR $\gamma \mathrm{t}$ expression and hence ROR $\gamma \mathrm{t}$ target genes expression e.g. $I L-17 a, I L-17 f$, and $I L-23 r$ [187] (Fig. 3-1). Therefore, the expression of T-bet during the development of HP may not only influence the development of a Th1 response but also a Th2 and Th17 response. In this chapter we will identify whether a deficiency in T-bet results in the development of a Th2 or Th17 response in HP and how that affects granuloma development and fibrosis.

The role of T-bet in different infectious and organ specific autoimmune disease models has been addressed in several studies [188-196]. Since Th1 cells are important for clearance of intracellular pathogens and T-bet is required for Th1 differentiation, deletion of T-bet in mice renders them susceptible to a wide range of intracellular pathogens e.g. Mycobacterium tuberculosis [188], Leishmania major [195], Staphylococcus aureus [189], and Salmonella typhimurium [190]. However, T-bet also may play a role in autoimmune diseases. T-bet KO mice are resistant to the development of several autoimmune diseases e.g. experimental autoimmune encephalitis (EAE) [191, 196], arthritis [192], and type I diabetes [193, 194]. Furthermore, T-bet KO mice infected with $S$. mansoni showed increase in disease severity accompanied by increase in Th17 cytokines [169]. Additionally, in T. cruzi mouse infection model there was an increase in the percentage of Th17 cells from the spleens of T-bet KO mice [170]. Several studies demonstrated the role of T-bet in fibrosis. T-bet $\mathrm{KO}$ mice treated with bleomycin showed an increase in lung and skin collagen content compared to WT mice [64, 65]. The goals of the studies are to determine the cytokines required for IFN $\gamma$ expression from neutrophils during HP and the effect of T-bet on disease severity and Th17 response during HP.

\title{
MATERIALS AND METHODS
}

\author{
Animals \\ Female C57BL/6J WT and T-bet KO mice were purchased from Jackson \\ Laboratories (Bar Harbor, ME) and used in experiments between 6-10 weeks of age. \\ Sterile micro-isolator cages with sterile food and water ad libitum were used for animal \\ housing. All the protocols were approved by the Institutional Animal Care and Use \\ Committee (IACUC), the University of Tennessee Health Science Center. All animals \\ were maintained by the Division of Comparative Medicine at the University of Tennessee \\ Health Science Center according to the guidelines of the animal welfare act.
}




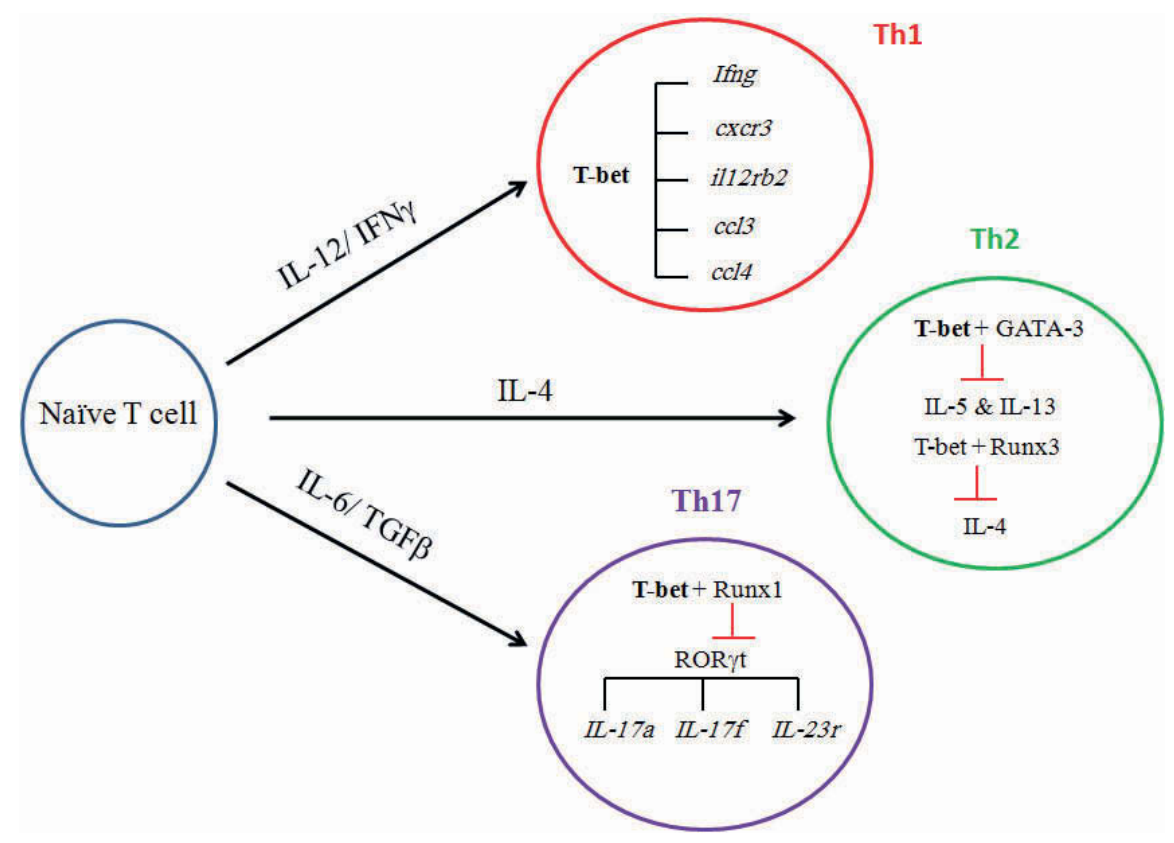

Figure 3-1. Role of T-bet in differentiation of different subsets CD4 ${ }^{+}$helper T cells.

In Th1cells, T-bet induces IFN $\gamma$, CXCR3, CCL3, CCL4, and IL-12R $\beta 2$ gene expression. T-bet inhibits Th2 differentiation by sequestering GATA-3 from binding to its specific promoters. T-bet inhibits differentiation of Th17 through binding to transcription factor Runx-1 inhibiting expression of ROR $\gamma \mathrm{t}$. (Adapted with permission Lazarevic, V. and L.H. Glimcher, T-bet in disease. Nat Immunol, 2011. 12(7): p. 597-606 [181].) 


\section{Spleen Cell Isolation and Stimulation}

Single cell suspension from spleen was prepared by digesting spleen tissue in a Stomacher ${ }^{\circledR} 80$ tissue processor. RBCs were lysed and the cell suspension was washed with sterile PBS. In a 96-well plate, $5 \times 10^{5}$ cells/well were stimulated with media alone, $5 \mu \mathrm{g}$ lyophilized SR, $5 \mu \mathrm{g}$ lyophilized SR in the presence of anti-IL12p40 $(10 \mu \mathrm{g} / \mathrm{ml})$, anti-IL-15 $(10 \mu \mathrm{g} / \mathrm{ml})$, anti-IL18 $(10 \mu \mathrm{g} / \mathrm{ml})$ individually or in combination. After 24 hours, culture supernatants were collected to measure IFN $\gamma$ concentration using ELISA.

\section{Neutrophil Cell Sorting and Stimulation}

A cell sorter (BD FACSAria) was used to sort lung neutrophils from C57BL/6J mice. Mice were exposed to $225 \mu \mathrm{g}$ SR one time; and sacrificed 18 hours post-exposure. A single cell suspension of lung cells was prepared and the cells incubated with fluorchrome labeled antibodies to the following cell surface markers: CD45, CD11b, Gr-1, and NK1.1. Neutrophils were sorted as (CD45 $\left./ \mathrm{CD} 11 \mathrm{~b}^{+} / \mathrm{Gr}-1^{\mathrm{hi}} / \mathrm{NK} 1.1^{-}\right)$and plated at $7.5 \times 10^{5}$ cells/well in a 96-well plate. The cells were stimulated with IL-12 $(10 \mathrm{ng} / \mathrm{ml})$, IL-18 $(20 \mathrm{ng} / \mathrm{ml})$, and or IL-23 $(20 \mathrm{ng} / \mathrm{ml})$ individually or in combination. After overnight incubation, the culture supernatants were collected to measure IFN $\gamma$ concentration by ELISA.

\section{RNA Extraction and Real-Time PCR}

\section{RNA Extraction}

Total RNA was extracted from one lung lobe from individual mice using Trizol (Invitrogen, Carlsbad, CA). Contaminating genomic DNA was removed by treating the RNA samples with DNase (Turbo DNA free, Ambion, Austin, TX). RNA concentration was determined by measuring the absorbance of UV light at 260nm, and 280nm using Ultrospec 2000 spectrophotometer (Pharmacia Biotech, Piscataway, NJ). The concentration of RNA $(\mu \mathrm{g} / \mathrm{ml})$ was calculated by multiplying the absorbance (A260), the dilution factor and $40 \mu \mathrm{g} / \mathrm{ml}$.

To determine the quality of the RNA $1-2 \mu$ gs were loaded on a $4 \%$ agarose gel and analyzed for the presence of $18 \mathrm{~S}$ and $28 \mathrm{~S}$ ribosomal RNA subunits as indicator of intact RNA.

\section{Real-Time PCR}

One $\mu \mathrm{g}$ of RNA was reverse transcribed into cDNA using Transcriptor First Strand cDNA synthesis kit (Roche Applied Science, Indianapolis, IN). Each PCR cycle was run using a LC480 light cycler with and activation step at $95^{\circ} \mathrm{C}$ for 5 minutes, denaturation at $94^{\circ} \mathrm{C}$ for $10 \mathrm{sec}$, annealing for 30 seconds at $60^{\circ} \mathrm{C}$ and extension at $72^{\circ} \mathrm{C}$ for 10 seconds. The reaction was amplified for 50 cycles. The housekeeping gene HPRT 
was used to normalize mRNA levels and $\Delta \Delta \mathrm{ct}$ method was used to calculate fold increase over the unexposed controls. All primers and probes were chosen from online software Universal Probe Library available at $<$ http://www.roche applied science.com/sis/rtpcr/upl/index.jsp $>$.

\section{Histology}

The perfused left lung lobe was harvested and fixed overnight in neutral buffered formalin at $4^{\circ} \mathrm{C}$. The lung tissue was embedded in paraffin and sectioned into $8 \mu \mathrm{m}$ sections and stained with Hematoxylin and Eosin (H\&E) by the Department of Pathology, UTHSC.

\section{Measurement of Lung Collagen Content}

The Sircol ${ }^{\mathrm{TM}}$ Collagen Assay (Biocolor Ltd., UK) was performed to measure collagen in the lungs of individual mice per manufacturer's instructions. The right lung lobe was homogenized in $0.5 \mathrm{M}$ acetic acid containing protease inhibitors and incubated for 24 hours at $4^{\circ} \mathrm{C}$ with shaking to extract acid soluble collagen. One $\mathrm{ml}$ of the homogenate was centrifuged at $15000 \mathrm{xg}$ for 1 hour and the supernatant removed and clarified by passage through a $0.22 \mu \mathrm{m}$ syringe filter. The standard curve was prepared from the collagen stock solution $(1 \mathrm{mg} / \mathrm{ml})$ using 2 -fold serial dilutions in deionized water The samples and standards were added to $1 \mathrm{ml}$ of sircoll dye followed by incubation at room temperature for 30 minutes with shaking. Following centrifugation at 10,000xg for 10 minutes, the unbound dye was aspirated and $1 \mathrm{ml}$ of alkali solution was added. Two hundred $\mu 1$ was transferred to a $96-w e l l$ plate and absorbance at $550 \mathrm{~nm}$ recorded. The concentration of the unknowns was calculated from the standard curve.

\section{Statistical Analysis}

Values were expressed as mean \pm S.D. For comparison of means between two populations of unpaired data, unpaired student's t-test was used. For comparison between more than two groups, One-way ANOVA followed by Tukey's post ANOVA test was used. GraphPad Prism was used to perform all tests. Differences were considered significant at $\mathrm{P}<0.05$. 


\section{RESULTS}

\section{Regulation of IFN $\gamma$ during HP}

Neutralization of IL-12p40 Inhibits IFN $\gamma$ Production from S. rectivirgula Stimulated Spleen Cells

Previous studies in our lab showed that neutrophils are the main source of IFN $\gamma$ following SR exposure [129]; however, it was unknown whether SR could induce IFN $\gamma$ directly or indirectly through induction of cytokines that stimulate IFN $\gamma$ production e.g. IL-12, IL-15, IL-18, and IL-23. Spleen cells were isolated from C57BL/6J mice and then stimulated with SR alone or in the presence of anti-IL-12p40 antibody (will neutralize both IL-12 and IL-23), anti-IL-15 antibody, and anti-IL-18 antibody individually or in combination. Twenty four hours later, culture supernatants were collected for measurement of IFN $\gamma$ using ELISA. As shown in Fig. 3-2, SR stimulated IFN $\gamma$ production from spleen cells and this stimulation was unaffected by the addition of antibody to IL-15 or IL-18 suggesting they do not play a role in SR stimulation of IFN $\gamma$. However, SR stimulation of IFN $\gamma$ was inhibited by neutralization of IL-12p40 alone or in combination with the other antibodies. This data suggested that SR mediates induction of IFN $\gamma$ from spleen cells via IL-12 and/or IL-23 since both cytokines share the IL-12p40 chain.

IL-12/IL-18 Combination but not IL-23 Combination with IL-12 or IL-18 Induces IFN $\gamma$ from Neutrophils

Previous studies in the lab had suggested that IL-18 in combination with IL-15 or IL-12 was able to stimulate IFN $\gamma$ production from neutrophils. However, the data in the previous section suggested that either IL-12 or IL-23 stimulated by SR could induce IFN $\gamma$. Therefore, we wanted to determine whether IL-23 alone or in combination with other cytokines could stimulate IFN $\gamma$ production. We sorted neutrophils from the lungs of mice exposed to SR one time and stimulated them with IL-12, IL-18, and IL-23 individually or in combination and measured IFN $\gamma$ production by ELISA. Only the combination of IL-12/IL-18 induced IFN $\gamma$ from neutrophils, while IL-23 individually or in combination with IL-12 or IL-18 did not (Fig. 3-3). These data suggested that neither IL-23 alone nor combination with other cytokines can stimulate IFN $\gamma$ production from neutrophils. Only IL-12 in combination with IL-18 can stimulate IFN $\gamma$ from neutrophils.

Role of IL-18 and T-bet in IFN $\gamma$ Production

Since our previous studies demonstrated that IL-18 in combination with either IL-12 or IL-15 stimulated IFN $\gamma$ from sorted neutrophils significantly more than any other combination, it suggests that IL-18 is critical for neutrophil IFN $\gamma$ production. Additionally, IL-18 stimulates IFN $\gamma$ through T-bet [197]. We hypothesized that SR uses the transcription factor T-bet for IFN $\gamma$ production. To test this hypothesis, spleen cells 


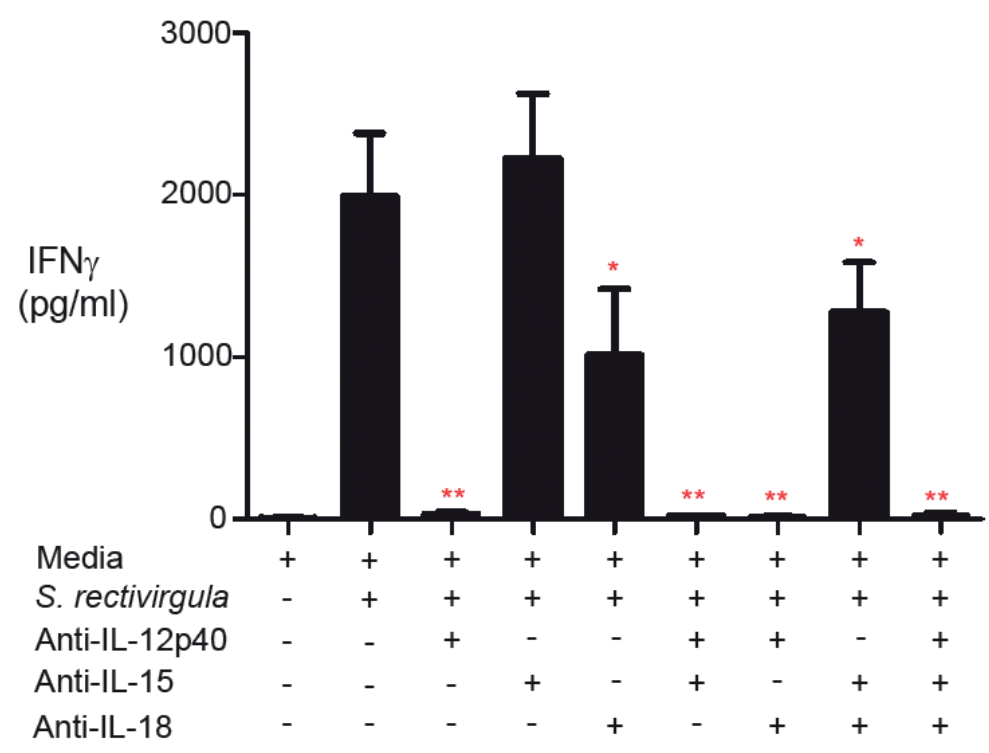

Figure 3-2. SR induces IFN $\gamma$ from spleen cells indirectly through cytokines.

Spleen cells isolated form C57BL/6J mice $(n=4)$ were stimulated for 24 hours with SR $(5 \mu \mathrm{g})$ alone or in the presence of anti-IL-12p40 antibody $(10 \mu \mathrm{g} / \mathrm{ml})$, anti-IL-15 antibody $(10 \mu \mathrm{g} / \mathrm{ml})$, anti-IL-18 anitbody $(10 \mu \mathrm{g} / \mathrm{ml})$ individually or in combination. Culture supernatants were collected and IFN $\gamma$ was measured by ELISA. Data were expressed as means $\pm \mathrm{SD}$. All cytokine combinations compared to each other using one way ANOVA followed by Tukey's post ANOVA test. ( ${ }^{*} \mathrm{P}<0.05$ and $* * \mathrm{P}<0.01$ significant difference from spleen cells stimulated with SR.) 


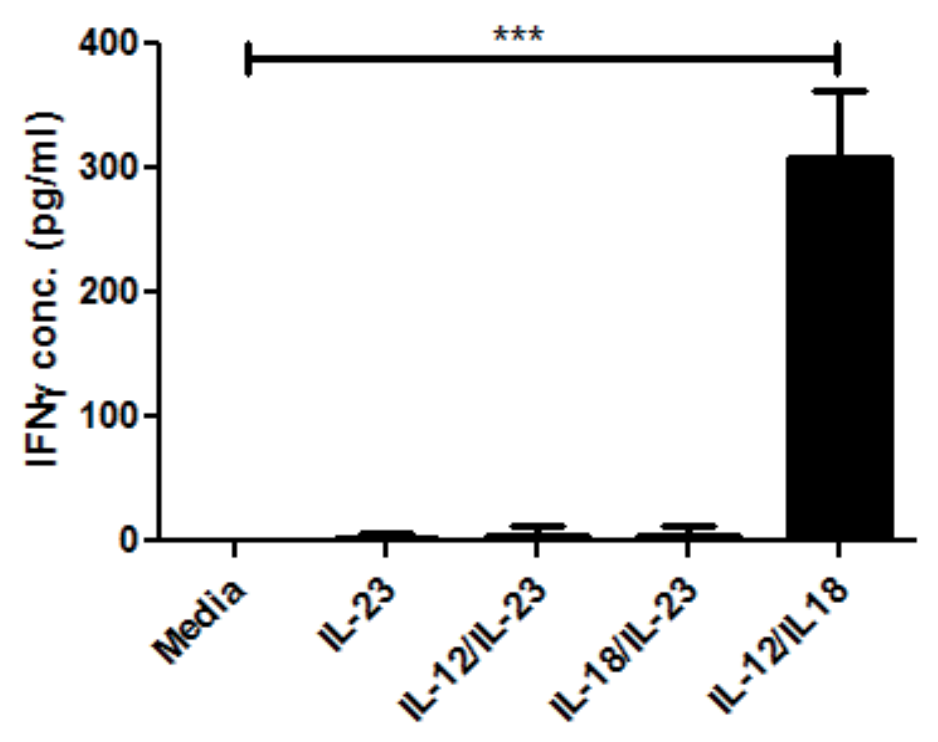

Figure 3-3. Neither IL-12 nor IL-23 alone stimulates IFN $\gamma$ production by neutrophils.

Neutrophils $\left(\mathrm{CD} 45^{+} / \mathrm{CD} 11 \mathrm{~b}^{+} / \mathrm{Ly}_{6} \mathrm{G}^{+} / \mathrm{NK} 1.1^{-}\right)$were sorted from the lungs of mice exposed one time with $225 \mu \mathrm{g}$ SR then sacrificed 18 hours post SR exposure. The neutrophils were stimulated with IL-12, IL-18, IL-23 individually or in combinations for 18 hours approximately. Culture supernatants were collected and IFN $\gamma$ was measured using ELISA. Data were expressed as means $\pm \mathrm{SD}$. Using unpaired student's t-test, means of control media were compared to IL-12/IL-18 induction. ( $* * * \mathrm{P}<0.0001$ significant difference from media control group.) 
from WT or T-bet KO mice were stimulated with SR. Culture supernatants were collected after 24 hour of stimulation and IFN $\gamma$ was measured using ELISA. The results demonstrated that following SR stimulation WT spleen cells produce a significant amount of IFN $\gamma$ compared to T-bet KO spleen cells (Fig. 3-4). These results suggested that $\mathrm{SR}$ induced IFN $\gamma$ from spleen cell in a T-bet dependent manner.

\section{Role of T-bet in HP}

T-bet Inhibits Development of the Th17 Response during HP

The previous section demonstrated the importance of T-bet in regulation of IFN $\gamma$ during acute HP. Regulation of IFN $\gamma$ is not the only function of T-bet. It is also involved in Th1cell recruitment through upregulation of the chemokine receptor CXCR3 and chemokines CCL3 and CCL4 [198, 199]. Additionally, T-bet is known to inhibit Th17 differentiation [187] and the predominant response during granulomatous and chronic HP was the Th17 response. Therefore, we examined the effect of T-bet on theTh17 response and disease severity during HP. Following three weeks of SR exposure, we found an increase in IL-17 mRNA expression in the lungs of T-bet KO mice compared to WT exposed mice. Also, there was a non-significant reduction in IFN $\gamma$ mRNA expression (Fig. 3-5).

Since we found an increase in IL-17 mRNA levels in T-bet KO mice, we measured IL-17 in BALF from T-bet KO and WT exposed mice using ELISA. Previous data in our lab showed an increase in IL-17 protein expression from BALF of T-bet KO mice compared to WT mice following three weeks of SR exposure. The upregulation of IL-17 mRNA and protein expression led us to determine the frequency of lung Th17 cells from T-bet KO and WT exposed mice. Previous studies in our lab showed a significant increase in the percentage of Th17 cells from the lungs of T-bet KO mice compared to WT exposed mice following ex vivo SR stimulation. In conclusion, these results demonstrated the inhibitory effect of T-bet on Th17 development during granulomatous HP.

\section{Tbet Controls Severity of HP}

As demonstrated in chapter 2, Th17 cells were associated with granuloma formation as well as the chronic phase of disease. In this chapter we showed an increase in the percentage of Th17 cells from T-bet KO exposed to SR. We hypothesized that the increase in Th17 cells in the T-bet KO mice would result in an increase in disease severity. WT and T-bet KO mice were exposed to SR for 3 weeks and granuloma formation in the lungs measured. The results demonstrated an increase in granuloma formation in T-bet KO mice exposed to SR compared to WT mice exposed to SR (Fig. 3-6).

The increase in granuloma formation in T-bet KO mice exposed to SR suggested 


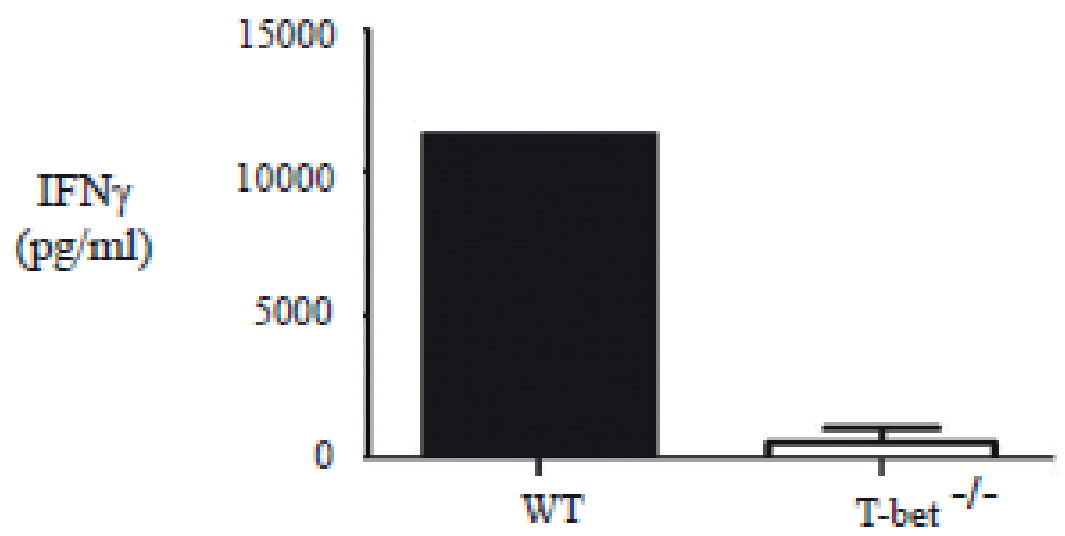

Figure 3-4. IFN $\gamma$ production from spleen is T-bet dependent following SR stimulation.

Spleen cells were isolated from WT and T-bet KO mice then stimulated with $10 \mu \mathrm{g}$ SR. Culture supernatant was collected following 24 hours incubation period. IFN $\gamma$ protein levels were measured using ELISA. 

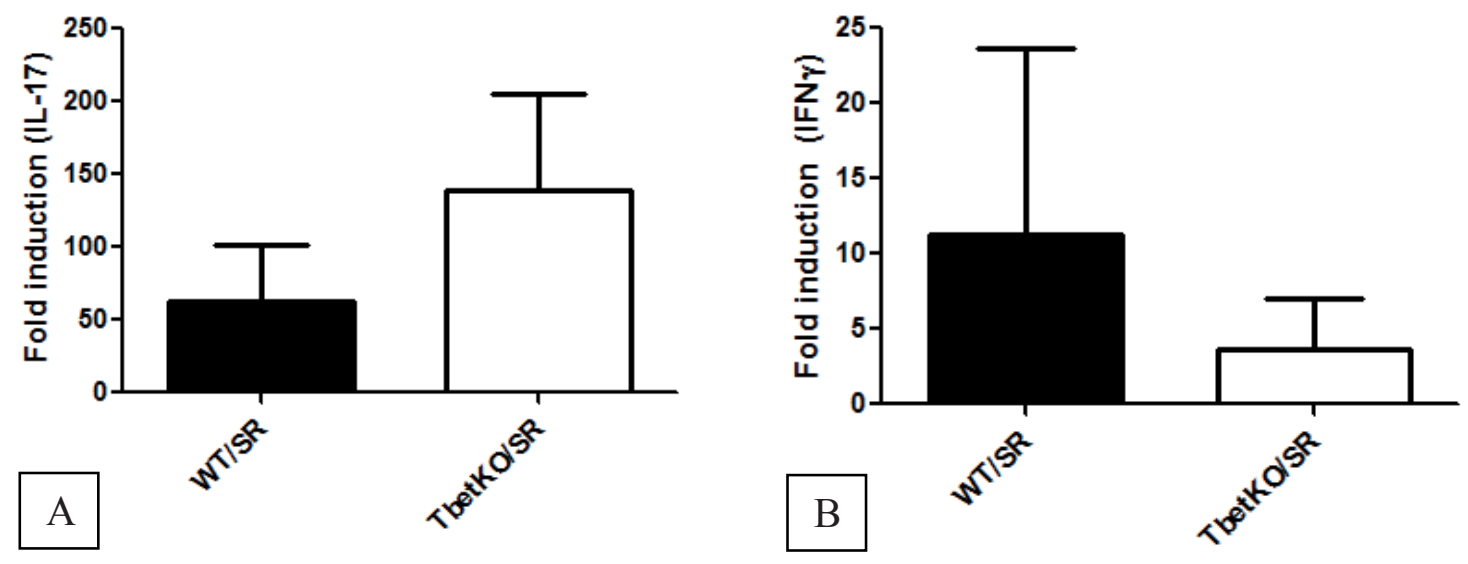

Figure 3-5. Real-time PCR showing expression levels of IL-17 and IFN $\gamma$ in lungs of WT and T-bet KO mice following 3 weeks of SR exposure.

Real-time PCR showing expression levels of IL-17 and IFN $\gamma$ in lungs of WT and T-bet KO exposed mice. WT and T-bet KO mice were exposed to $150 \mu \mathrm{g}$ SR for three weeks. RNA was extracted from one lung lobe then reverse transcribed to cDNA. Real-time PCR was performed using primers specific to IL-17 (Panel A) and IFN $\gamma$ (Panel B). Results were normalized to the house keeping gene HPRT and expressed as fold induction over unexposed mice. Results were expressed as mean \pm SD. Using unpaired student's t-test, means of both groups were compared showing a non-significant difference. 


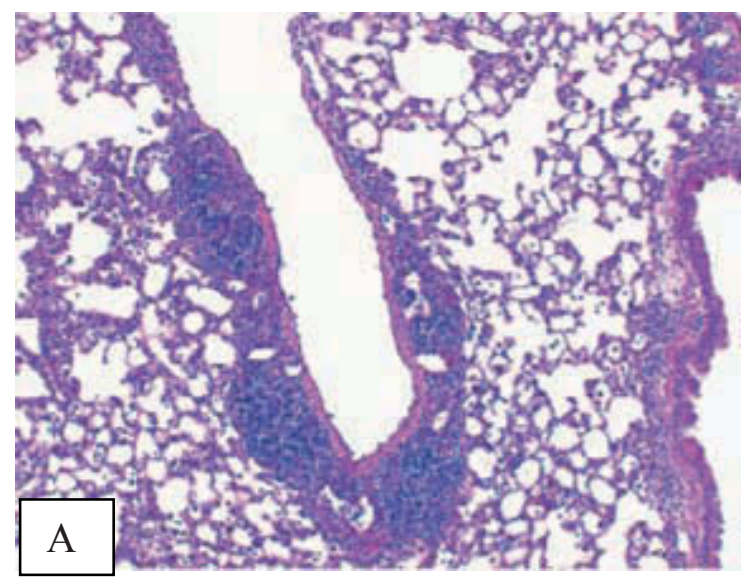

WT

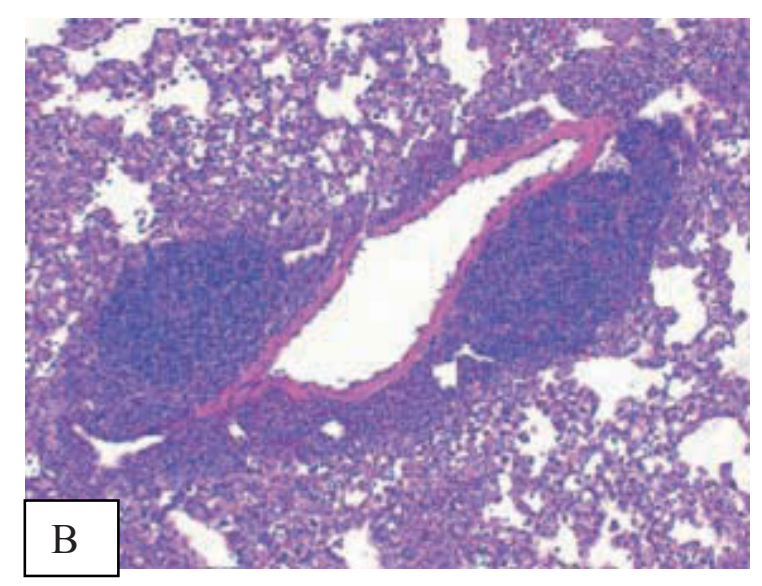

T-bet KO

Figure 3-6. Granuloma formation in WT and T-bet KO mice.

H\&E stained lung section from WT and T-bet KO mice exposed to $150 \mu \mathrm{g}$ SR for three weeks. (Original magnification 63X). Panel A represents lung section from WT SR exposed mouse. Panel B represents lung section from T-bet KO SR exposed mouse. 
to us there will be an increase in disease severity upon subsequent exposures. Using the Sircoll assay we measured collagen content as a measure of fibrosis in the lungs of mice exposed to SR for six weeks. The results demonstrated a significant increase in lung collagen content from T-bet KO exposed mice compared to WT exposed mice (Fig. 3-7). In conclusion, these results suggested that T-bet plays a protective role during HP, possibly by limiting Th17 cell generation.

\section{DISCUSSION}

The pathogenic role of IFN $\gamma$ during HP was addressed by Gudmundsson and Hunninghake [128]. They found that IFN $\gamma$ KO (GKO) mice did not develop granulomas following SR exposure for 3 weeks compared to WT exposed mice that developed granulomas. Furthermore, the replacement of IFN $\gamma$ in GKO mice via intraperitoneal injections of IFN $\gamma$ restores back granuloma formation in GKO SR exposed mice [128]. Those early studies suggested that the source of IFN $\gamma$ was Th1 cells however, that was never proven. Previous studies in our lab did not find a large percentage of Th1 cells during HP, rather these studies demonstrated that innate immune cell IFN $\gamma$ production was sufficient for disease. Additionally, one of the innate immune cells contributing to IFN $\gamma$ production was the neutrophil [129]. However, elucidation of the cytokines required for IFN $\gamma$ expression from neutrophils and/or during HP was not addressed. Several cytokines can stimulate IFN $\gamma$ from different immune cells and they may function in a T-bet dependent or independent manner. IL-2, IL-12, IL-15, IL-18, TNF $\alpha$ as well as LPS

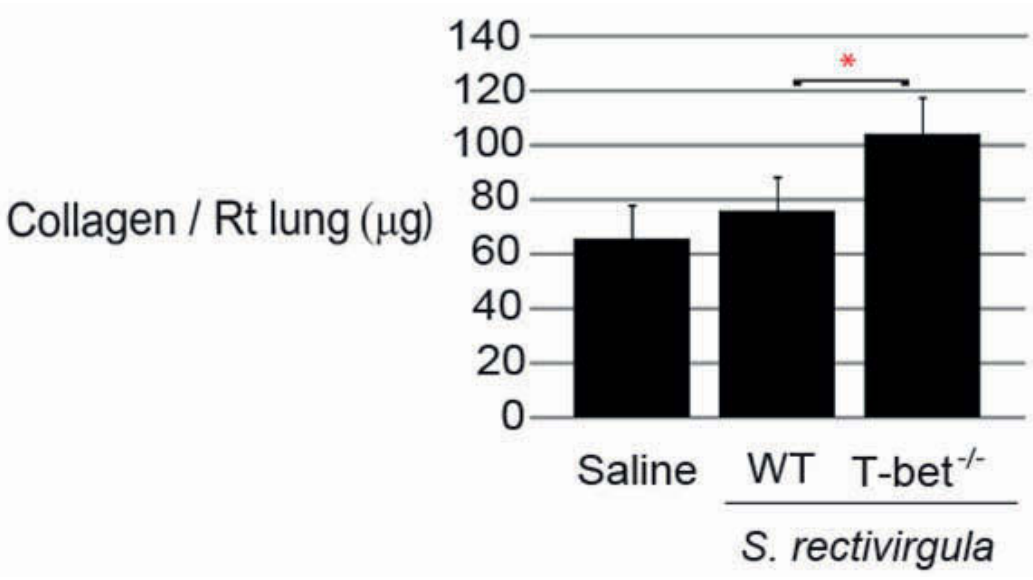

Figure 3-7. Increased collagen content in the lungs of T-bet KO mice during HP.

C57BL/6J or T-bet KO mice (5/group) were exposed to 150ug SR or saline for six weeks. The right lung lobe was removed from mice and collagen content was determined using sircoll assay. The results are expressed as amount of collagen/right lobe and represent the mean $\pm \mathrm{SD}$. Using unpaired student t-test, mean of WT exposed group was compared to mean of $\mathrm{T}$-bet $\mathrm{KO}$ exposed group. $(* \mathrm{P}<0.05$.) 
have all been demonstrated to stimulate IFN $\gamma$ from macrophages and neutrophils [182, $183,200]$. Our results found that neutrophils sorted from SR exposed mice (single exposure) produced IFN $\gamma$ in response to IL-18 in combination with IL-12 or IL-15, but not to any cytokine alone. It has been reported that alveolar macrophages isolated from HP patients produce IL-12 and IL-18 spontaneously without ex vivo stimulation. [114]. Therefore, these cytokines produced by AMs might be crucial for production of IFN $\gamma$ from neutrophils. In conclusion, the data demonstrated to us the importance of IL-12, IL-15, and IL-18 in production of IFN $\gamma$ from neutrophils during HP.

IL-18 can stimulate IFN $\gamma$ expression through T-bet [197], and so we asked whether SR induction of IFN $\gamma$ was also through T-bet Spleen cells isolated from a T-bet $\mathrm{KO}$ mouse did not produce IFN $\gamma$ following SR stimulation compared to WT spleen cells. These results suggested that neutrophil IFN $\gamma$ production may be dependent on T-bet; however, we were not successful in our attempts to demonstrate T-bet in neutrophils using intracellular staining. One problem with the experiment was not having a strong positive control such as a type of immune cells that express high levels of T-bet. A separate experiment would be to sort neutrophils from T-bet KO SR exposed mice to check the expression of IFN $\gamma$ in the absence of T-bet. In conclusion, the results from these studies revealed the importance of T-bet and combination of IL-18/IL-15 or IL-18/IL-12 in IFN $\gamma$ regulation during acute HP.

Our studies in chapter 2 found that HP was associated with a Th17 response rather than a Th1 or Th2 response. However, there was a small percentage of SR antigen specific Th1 cells in the lungs of exposed mice. Previous studies from our lab demonstrated that during HP, IFN $\gamma$ produced by Th1 cells is not necessary for granuloma formation this raises the question "what is the role of Th1 cells in the disease severity and regulation of Th17 response during HP?" To answer this question we used T-bet KO mice which lack the ability to mount Th1 cell responses. We exposed WT and T-bet KO mice for three and six weeks and examined the Th17 response, granuloma formation, and fibrosis in the lungs. We found an upregulation in IL-17 mRNA and protein expression following 3 weeks of SR exposure compared to WT exposed mice. In addition, there was an increase in the percentage of Th17 cells in the lungs following SR exposure compared to WT exposed mice. These results suggested a regulatory effect of T-bet on Th17 cell development and expression IL-17 expression. Several disease models demonstrated the regulatory effect of T-bet on Th17 effector cytokines. In the $S$. mansoni mouse infection model, T-bet KO mice showed an increase in Th17 cytokines compared to WT infected mice [169]. In another parasitic mouse infection model, T-bet KO mice develop a strong Th17 response in the spleens compared to WT mice following T. cruzi infection. Also there was an increase in the number of neutrophils in the blood [170].

The increase in Th17 cells during the granulomatous phase of the Tbet $\mathrm{KO}$ mice suggested to us that the disease was more severe and therefore we examined granuloma formation and fibrosis in the WT and T-bet KO mice. As expected following 3 weeks of SR exposure we found an increase in granuloma formation in T-bet $\mathrm{KO}$ mice compared to WT mice although we have not quantified it by measuring the area of the granulomas between the WT and Tbet KO groups. Since the morbidity of the disease is due to 
fibrosis, we measured the collagen content in the lungs of WT and T-bet KO mice and found an increase in collagen in the T-bet KO mice following 6 weeks of SR exposure. Taken together, the shift to stronger Th17 response in T-bet KO exposed mice correlated with increase in disease severity. These data suggested two roles for T-bet during HP. First, it suggested the protective role of T-bet. Second, the inhibitory effect of T-bet on Th17 development; however the exact mechanism by which T-bet inhibits Th17 development was not clear until recently. Lazarevic et al. showed that T-bet binds to Runx-1 transcription factor inhibiting expression of Th17 specific transcription factor (ROR $\gamma \mathrm{t}$ ) and hence its target genes $I L-17 a, I L-17 f$, and $I L-23 r$ [187].

The data from these studies demonstrated the role of T-bet in disease severity and its effect on Th17 development during HP. Our results do not identify whether T-bet expression by an innate or adaptive immune cell is responsible for the inhibition of Th17 development. The direct experiment to fulfill this aim would require an adoptive transfer experiment. WT or T-bet $\mathrm{KO} \mathrm{CD} 4^{+} \mathrm{T}$ cells from naïve mice will be injected into RAG-2 $\mathrm{KO}$ mice. Both groups of mice will be exposed to SR for three weeks (3 days/week). We might expect two scenarios. The first scenario, if both groups developed an equal percentage of Th17 cells in the lungs, the T-bet expressed by $\mathrm{T}$ cells does not play a role in Th17 development during HP. The second scenario, if the frequency of Th17 cells is more in the group that received T-bet KO T cells than the one received WT CD4 ${ }^{+} \mathrm{T}$ cells therefore, T-bet regulates development of Th17 in T cell intrinsic manner. In T. cruzi mouse infection model, T-bet regulates Th17 development in T cell intrinsic manner. The authors showed the adoptive transfer of naïve T-bet $\mathrm{KO} \mathrm{CD} 4^{+} \mathrm{T}$ cells into RAG-2 $\mathrm{KO}$ enhance expression of IL-17 from $\mathrm{CD}^{+}{ }^{+} \mathrm{T}$ cells more than WT transfer to RAG-2 KO mice [170].

The role of T-bet in different infectious and organ specific autoimmune disease models has been addressed in several studies [188-196]. In the $S$. mansoni mouse infection model, T-bet KO mice showed an increase in disease severity accompanied by upregulation in Th17 cytokines compared to WT infected mice [169]. T-bet KO mice infected with schistosome egg antigen in CFA (SEA/CFA) developed larger liver granulomas infiltrated with neutrophils compared to WT infected mice [169]. Furthermore, there was an increase in Th17 effector cytokines (IL-17, IL-21, and IL-22) as well as neutrophil chemokines CXCL1 and CXCL2 [169]. In another parasitic mouse infection model, T-bet KO mice develop a strong Th17 response in the spleens compared to WT mice following T. cruzi infection. Also there was an increase in the number of neutrophils in the blood [170]. T-bet does not regulate only granuloma formation and Th17 response in disease, but also it can regulate fibrosis. For example, in bleomycin-induced lung injury mouse model, T-bet KO mice were characterized by an increase in lung collagen content as a marker for fibrosis compared to WT mice [64].

Since T-bet KO mice exposed to SR were characterized by an increase in Th17 cells as well as an increase in disease severity, the next experiment is to figure out if Th17 cells are required for granuloma formation. To address this question, previous studies in our lab used IL-6 KO mice. IL-6 is one of the cytokines required for Th17 development [16]. When the lung cells from these mice were culture in vitro with 
PMA/Ionomycin and intracellular cytokine staining was used to phenotype these cells, it was determined that the absence of IL-6 resulted in a significant decrease in Th17 percentage and increase in Th1 percentage in the lungs of IL-6 KO exposed mice compared to WT exposed mice. However, there was no difference in granuloma formation between WT and IL-6 KO exposed mice as examined by H\&E staining. These data indicated that Th17 were not required for granuloma formation and Th1 response was sufficient for granuloma formation indicating that both Th1 and Th17 may coexist during HP. Several experimental disease models showed the coexistence of both Th1 and Th17 cells during the disease progression. In EAE, both Th1 and Th17 are infiltrating the CNS [178] and antigen specific Th1 or Th17 generated in vitro adoptively transfer the disease into naïve mice [179]. In T. cruzi mouse infection model, normal Th1 response developed upon antigen challenging along with strong Th17 response [170]. In experimental autoimmune uveitis as a model for human uveitis, either Th1 or Th17 can cause the disease [177]. The cooperation between both types of $T$ cells was demonstrated in EAE model where Th1 cells collaborate with Th17 cells by facilitating the entry of Th17 into CNS [180]. In the same line of evidence, during HP either Th1 or Th17 may cause disease severity and the tipping balance point between both populations might regulate the disease severity. 


\section{CHAPTER 4. EFFECT OF TLR2 AND 9 IN APCS ON TH17 CELL POLARIZATION AND EFFECTOR FUNCTION}

The results from chapters 2 and 3 demonstrated that the Th17 response was predominant over the Th1 response during HP and may contribute to disease severity. Consequently, we were looking for other factors that might control the Th17 response in our model. Activation of antigen specific T cells depends on interactions with innate immune cells which in turn are activated by the presence of a microbe. The innate immune cells recognize microbes using Toll like receptors (TLRs) expressed on the cell surface and in the cytoplasm. Previous results in our lab showed that the frequency of Th17 cells was significantly less in the lungs of TLR 2/9 double Knockout (DKO) mice compared to WT mice during HP. The aim of this chapter is to determine whether TLRs 2 and 9 expressed by antigen presenting cells contributes to the Th1 or Th17 response during HP.

\section{INTRODUCTION}

\section{Antigen Processing, Presentation, and Recognition Processes}

As introduced in chapter one, naïve T cell differentiation takes place in the lymph nodes where DCs are presenting specific antigen to naïve T cells. DCs provide naïve T cells with three signals. The culmination of the three signals ultimately leads to $\mathrm{T}$ cell differentiation into Th1, Th2, or Th17. The process of T cell differentiation requires the combination of sequential processes that take place at different anatomical sites. The first process occurs at the site of infection where immature DCs, that have a high phagocytic activity, phagocytose the microbial pathogen. Consequently, DCs undergo a maturation program allowing them to migrate to the regional lymph nodes and present the microbial antigen to naïve $\mathrm{T}$ cells. Immature DCs express a wide range of phagocytic receptors involving lectins and scavenger receptors with a very high phagocytic activity; however, once they get exposed to an inflammatory signal e.g. TLR ligand or inflammatory cytokine, they undergo a program of maturation. Mature DCs are mainly functional as antigen presenting cells. There are several events that occur during the DC maturation process. For example, downregulation of their phagocytic capacity, translocation of MHCII from the lysosomal compartment to plasma membrane increasing the expression of MHCII on the cell surface, upregulation in MHCI and costimulatory molecules expression as well [5]. All of these characteristics endow mature DCs with the capacity to become the most efficient APCs for priming naïve T cells at the lymph nodes while macrophages are inherently oriented towards activation of effector T cells already present at the site of inflammation. Also, the macrophages lysomal compartment is more adapted toward complete clearance of the microorganism [201].

Following phagocytosis, antigen presentation and antigen recognition processes take place. The recognition of antigens by $\mathrm{T}$ cells is a crucial process both in terms of 
initiating the adaptive immune response at the lymph nodes as well as maintaining the effector phase of the immune response at the site of infection. Antigen recognition by $\mathrm{T}$ cells as mentioned earlier is preceded by two subsequent processes; antigen processing and presentation. Antigen processing begins with a form of internalization in which the immune cells engulf the cargo (microorganism or particulate) as one mechanism of host defense. Phagocytic innate immune cells such as macrophages and dendritic cells are capable of phagocytosis as a mode of internalization. They are also capable of performing other processes of internalization including macropinocytosis and clathrin and non clathrin-mediated endocytosis [201]. Phagocytosis, which was discovered by Elie Metchnikoff in 1882, is an internalization process initiated by binding of a microorganism or particle to phagocytic receptors on cell surface. This binding triggers a cascade of signaling events inducing cytoskeleton remodeling which allows membrane movement; consequently, engulfment of the cargo and phagosome formation occurs. The phagosome is the organelle that contains the cargo. It undergoes a series of events including fission and fusion with endosomes and lysosomes to ultimately generate phagolysosome, a process called phagosome maturation [202-204]. The lysosomes contain a harsh acidic environment due to vacuolar ATPase assembly which is pumping in protons from the cytoplasm. Also it contains proteolytic enzymes for protein degradation and peptide generation. The acidic $\mathrm{pH}$ denatures the proteins to be easily degradable by proteolytic enzymes [205] and these conditions favor the generation of antigenic peptides. In the endoplasmic reticulum (ER), the newly synthesized MHCII is associated with an invariant chain (Ii) that leads MHCII from the ER to the endocytic pathway. The invariant chain is cleaved by the proteases in the endosome leaving Class II-associated Ii derived peptide (CLIP) in the MHCII binding groove. Finally, CLIP is exchanged by the antigenic peptide with the help of a MHCII like chaperone in the presence of acidic $\mathrm{pH}[201]$.

\section{Effect of TLRs Signaling on Phagocytosis}

Toll-like receptors (TLRs) belong to a family of germline-encoded pattern recognition receptors (PRRs). They detect conserved microbial structures called pathogen-associated molecular patterns (PAMPs). TLRs are considered as type I transmembrane glycoprotein receptors made of three domains. The N-terminal ectodomain which is responsible for recognition of PAMPs; a transmembrane domain; and the C-terminal intracellular domain that shows homology with IL-1 receptor for that it is called Toll/IL-1 receptor domain (TIR). The TIR domain interacts with different adapter proteins such as MyD88 to activate downstream signaling. The stimulation of MyD88 leads to activation of NFkB signaling and the MAPK (mitogen-activated protein kinase) pathway which is important for expression of cytokines and chemokines such as IL-6, TNF $\alpha$ and IL-8. Humans and mice have 10 and 13 different form of TLRs respectively. TLRs 1-9 are conserved in both species with the absence of TLRs 11, 12, and 13 in humans and non-functional TLR10 in mice. TLRs are further classified into 2 subgroups based on their localization. For instance, TLRs 1, 2, 4, 5, 6, and 11 are expressed on the cell surface, while TLR 3, 7, 8, and 9 are expressed in intracellular 
compartments such as endosomes, lysosomes, and endoplasmic reticulum for recognition of nucleic acids [206].

Although TLRs have been known for their induction of cytokine expression, several studies examined the effect of TLR signaling on phagocytosis. Blander and Medzhitov demonstrated that TLRs regulate phagocytosis at different levels [207]. The authors showed a defect in internalization of E. coli, S. aureus, and heat-killed S. typhimurium by TLR2/ 4 DKO and MyD88KO bone marrow-derived macrophages compared to WT counterparts. Furthermore, there was incomplete colocaliztion of GFP labeled $E$. coli with a marker for late endosomes and lysosomes indicating a defect in phagosome maturation [207]. Finally, inhibition of p38 mitogen-activated protein kinase (MAPK) impaired the ability of macrophages to phagocytose E. coli. [207]. In conclusion, TLR signaling affects the phagocytosis process.

It was also examined if TLRs signaling can affect antigen presentation as a subsequent process to phagosome maturation [208]. They found that a phagosome that carries a microbial cargo, i.e. pathogen binding to TLR, is able to mature to endosomal and lysosomal compartments and the microbial peptides will have access to MHCII for peptide-MHCII loading; hence their transportation to cell surface. On the other hand, phagocytosis of apoptotic cells generate peptides in phagolysosomal compartment; however, the peptides do not have access to MHCII and consequently the formation of peptide-MHCII complex is inhibited [208]. In summary, the type of the cargo packed with TLR in the phagosome can dictate the fate of the phagosome.

Other studies have shown the effect of TLRs on phagocytosis in several bacterial infections. For instance, in pneumococcal infection, TLR9 and MyD88 deficient alveolar and bone marrow-derived macrophages develop defects in internalization as well as bacterial killing compared to WT counterparts [209] showing that TLR signaling is indispensible for defense against Streptococcus pneumoniae. Furthermore, macrophages from TLR2 KO and MyD88 KO mice were impaired in L. monocytogenes uptake [210] After intraperitoneal infection, TLR2KO and MyD88KO peritoneal macrophages have low numbers of L. monocytogenes compared to WT [210]. In conclusion, TLRs can affect the phagocytosis process in different bacterial infection models.

\section{Effect of TLRs Signaling on Costimulatory Molecules and Cytokine Expression}

As mentioned earlier, both ligation of costimulatory molecules and the appropriate cytokine milieu are required for differentiation of naïve T cells. Several studies showed the effect of TLRs not only on phagocytosis but also on the expression of cytokines and costimulatory molecules [211-213]. For instance, it was demonstrated that peptidoglycan-associated lipoprotein (PAL) of L. pneumophila is responsible for induction of proinflammatory cytokines IL- 6 and TNF $\alpha$ from peritoneal macrophages through TLR2. Furthermore, the production of IL- 6 and IL-12 was enhanced by costimulation with PAL and CpG ODN as a synthetic stimulus for TLR9. These data showed that TLRs2 and 9 induce proinflammatory cytokines from peritoneal 
macrophages in a cooperative manner [211]. Additionally, PAL induced cell surface expression of MHCI and II molecules and costimulatory molecules CD40, 80, and 86. Also, costimulation with PAL and CpG ODN increased CD80, CD40, and MHCII expression but not CD86 and MHCI expression when compared to PAL stimulation alone [211]. In another study, Helicobacter pylori stimulated production of IL-6, IL-8, IL-10, and IL-12 production from immature human DCs in a dose-dependent manner. There was also an increase in mean fluorescence intensity (MFI) of CD80, CD83, and CD86 as well as MHCII [212]. Different species of lactobacillus induce different cytokines such as IL-6, IL-10, IL-12, and TNF $\alpha$ as well as upregulation of CD86 and MHCII [213]. In conclusion, signaling through different types of TLRs affects the expression of costimulatory molecules and cytokines.

\section{The Role of TLRs in Innate and Adaptive Immunity during HP}

Previous studies in our lab have shown the crucial role of MyD88 in neutrophil recruitment and chemokine expression during the innate phase of HP [127]. Our lab wanted to identify the TLRs upstream of MyD88 that interact with SR. Since SR is a gram-positive bacterium, in vitro studies demonstrated that TLR2 was the candidate PRR used by SR [127]. We used TLR2 KO mice to investigate whether SR interact with TLR2 to effect neutrophil recruitment and chemokine expression. TLR2 KO mice had a decrease in neutrophil recruitment although it did not reach statistical significance compared to the WT mice exposed to SR. CXCL2 was significantly downregulated in TLR2 KO adherent splenic cells following in vitro SR stimulation when compared to WT counterparts [127]. These data demonstrated that while TLR2 contributes to neutrophil recruitment there is another TLR(s) that also plays a role. One other candidate is TLR9 which is expressed intracellularly in endosomes to recognize $\mathrm{CpG}$ motifs in bacterial DNA. TLR2/9 DKO mice were generated by the lab to investigate if TLR9 cooperates with TLR2 to control neutrophil recruitment. Previous data in our lab showed that TLR2/9 DKO mice had a significant reduction in neutrophil recruitment as well as neutrophil chemokine expression following SR exposure for 6 hours as compared to WT counterparts. Also, there was a significant reduction in neutrophil chemokine (CXCL2) in TLR2/9DKO mice following three weeks of SR exposure. In conclusion, both TLRs 2 and 9 affect neutrophil recruitment during HP through regulation of neutrophil chemokine (CXCL2).

Previous data in our lab also demonstrated that following three weeks of SR exposure, the frequency of Th17 cells was higher in the lungs of WT mice compared to TLR2/9 DKO mice. A multitude of factors can control this phenomenon. First, the inability of DCs in the lymph nodes to polarize naïve T cells efficiently to effector Th17 cells due to a defect in costimulatory molecules and/or cytokine expression by DCs. Second, the efficiency of Th17 polarization in the lymph nodes might be equal in both WT and TLR2/9 DKO mice, but there is a defect in the process of effector Th17 cell migration from lymph nodes to the lungs of DKO mice. Finally, polarization and migration of Th17 cells might be equally efficient in both WT and TLR2/9 DKO mice; however, there could be a defect in antigen presentation capability of antigen presenting 
cells (APCs) in the lungs of TLR2/9 DKO mice where they are unable to stimulate Th17 cells to produce IL-17 as an effector cytokine. In conclusion, one or a combination of these scenarios might occur. The aim of this chapter is to determine whether antigen processing or presentation by TLR2/9 deficient innate immune cells is responsible for the decrease in $\mathrm{IL}-17^{+} \mathrm{CD} 4{ }^{+} \mathrm{T}$ cells in the lungs.

\section{METHODS}

\section{Animals}

Female C57BL/6J WT and MyD88KO mice were purchased from Jackson Laboratories (Bar Harbor, ME) and used in experiments between $6-10$ weeks of age. TLR9 KO mice were originally obtained from Dr. A.K Yi, (Dept. of Pediatrics, University of Tennessee Health Science Center) and the colony was maintained at UTHSC. The TLR9 KO mice were crossed with TLR2 KO mice to generate TLR2/9DKO mice which were bred at the University of Tennessee Health Science Center and were used in at least $5^{\text {th }}$ generation backcross. Sterile micro-isolator cages with sterile food and water ad libitum were used for animal housing. All the protocols were approved by the Institutional Animal Care and Use Committee (IACUC), the University of Tennessee Health Science Center. All animals were maintained by the Division of Comparative Medicine at University of Tennessee Health Science Center according to the guidelines of the animal welfare act.

\section{Antigen Presentation Assay}

\section{Isolation of $\mathrm{CD} 4^{+} \mathrm{T}$ Cells}

WT and TLR2/9 DKO mice were exposed to 150ug lyophilized SR for three weeks. Mice were sacrificed 16-18 hrs following last exposure. BAL was performed using $10 \mathrm{ml}$ of sterile PBS-EDTA $(0.1 \mathrm{mM})$ to remove most of the alveolar macrophages and neutrophils from the air space. Lungs were perfused using $10 \mathrm{ml}$ of sterile PBS and then harvested for collagenase/DNase digestion. Single cell suspension was prepared using stomacher. Lung total $\mathrm{T}$ cells were enriched by an adherence step where cell suspension was cultured in $100 \mathrm{~mm}$ petri-dishes. Following 2 hours of incubation at $37^{\circ} \mathrm{C}$, non-adherent cells were collected and the plate was washed once with PBS. CD4 ${ }^{+} \mathrm{T}$ cells were enriched by negative selection method (BD IMag). The purity was usually between $70-80 \% \mathrm{CD}^{+} \mathrm{T}$ cells.

\section{Isolation of Adherent Cells}

One day before the experiment, spleen cells were harvested from WT and TLR2/9 DKO mice and single cell suspension were prepared using a Stomacher. The cell suspension from each genotype was cultured in a $100 \mathrm{~mm}$ petri-dish and incubated at $37^{\circ} \mathrm{C}$ for 2 hours then adherent cells were harvested. In a 6 -well plate, $5 \times 10^{6}$ adherent 
spleen cells were plated and stimulated with $8 \times 10^{7}$ CFU of heat-killed SR (HKSR) for 24 hours. Another group of adherent spleen cells were incubated for 24 hours without HKSR. The reason behind the 24 hours incubation was to give time for the adherent spleen cells to phagocytose, process, and present SR antigen. Next day (the day of the experiment), adherent spleen cells were harvested by incubating them with PBS for 15 minutes at $4^{\circ} \mathrm{C}$ and then washed with media for 3 times to get rid of free unphagocytosed SR.

Co-Culturing $\mathrm{CD}^{+}{ }^{\mathrm{T}}$ Cells with Unstimulated or Stimulated Adherent Spleen Cells

In a 96-well plate, $\mathrm{CD}^{+} \mathrm{T}$ cells and adherent spleen cells were cocultured at 1:1 ratio. Fig. 4-1 summarizes the antigen presentation assay.

\section{Generation of Bone Marrow Derived Macrophages and Dendritic}

Preparation of Bone Marrow Derived Macrophages (BMDMs) Cell Culture Medium

L-929 culture conditioned medium (LCCM) was used as the source of M-CSF (Macrophage Colony Stimulating Factor) to generate macrophages from bone marrow in vitro. The L-929 murine fibroblast cell line was obtained from Dr. Ted Strom (VAMC). The cells were maintained in DMEM containing 10\% heat inactivated FCS, Glutamine $(2 \mathrm{mM})$, and penicillin/streptomycin $(100 \mathrm{U} / \mathrm{ml}$ and $100 \mu \mathrm{g} / \mathrm{ml})$. After $4-5$ days, the culture supernatant was collected and stored at $-20^{\circ} \mathrm{C}$, and the cells were split into flasks with a larger surface area. 4-5 days later the supernatant was collected and pooled with the previous collection. After collecting 1-2 liters of supernatant, the whole preparation was thawed once then aliquoted into $50 \mathrm{ml}$ tubes and stored at $-80^{\circ} \mathrm{C}$. The complete conditioned cell culture medium used for generation of bone marrow-derived

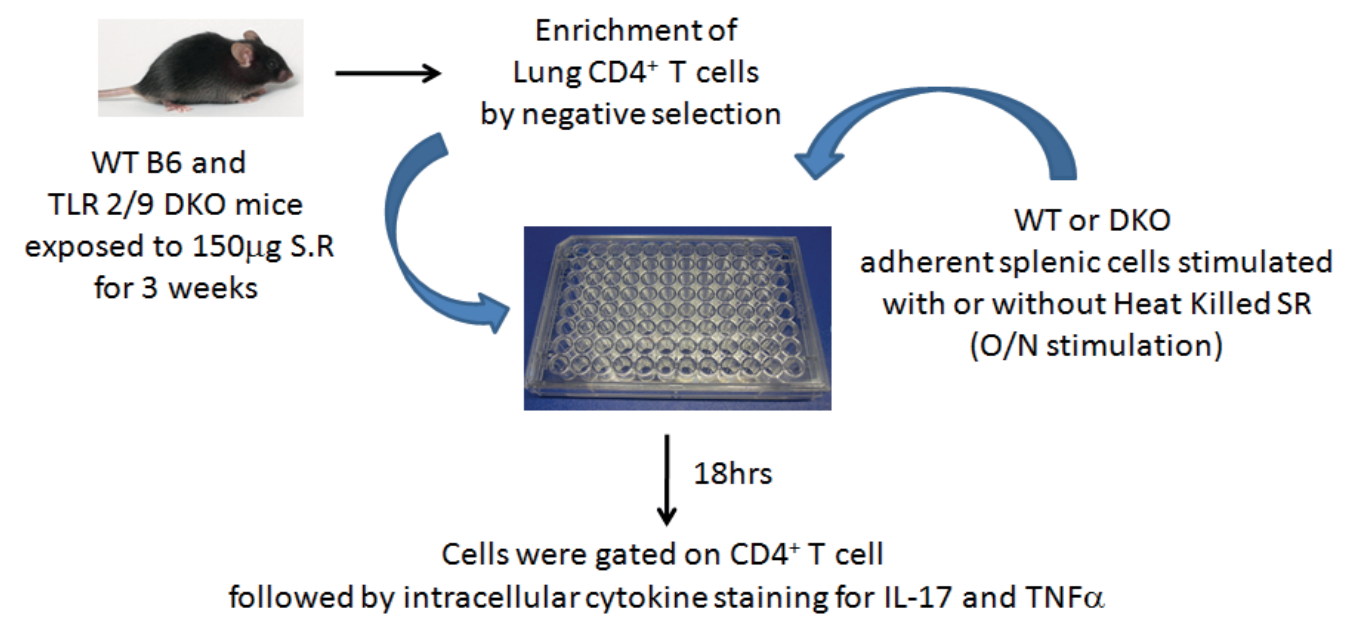

Figure 4-1. Steps for setting up antigen presentation assay. 
macrophages contained DMEM (Cellgro, Mediatech Inc., Manassas, VA) supplemented with $15 \%$ heat inactivated FCS, Glutamine $(2 \mathrm{mM})$, Penicillin/Streptomycin $(100 \mathrm{U} / \mathrm{ml}$ and $100 \mu \mathrm{g} / \mathrm{ml}$ ), $50 \mu \mathrm{g} / \mathrm{ml}$ gentamicin (GIBCO cell culture Invitrogen; Carlsbad, CA) and 30\% L-929-cell-conditioned medium (LCCM).

\section{Generation of BMDMs}

Bone marrow cells were harvested from the tibias and femurs of C57BL/6J, TLR2/9DKO, and MyD88 KO mice by flushing with sterile PBS. The RBCs were lysed and the bone marrow cells were washed and suspended in $15 \mathrm{ml}$ of complete conditioned medium. The cell suspension was plated in $100 \mathrm{~mm}$ non-tissue culture petri-dishes and incubated at $37^{\circ} \mathrm{C}$. The next day, non-adherent cells were collected, counted, and plated at $5 \times 10^{6}$ cells $/$ dish in a $100 \mathrm{~mm}$ petri-dish with $15 \mathrm{ml}$ of complete conditioned medium. On day 3 , half of the media was removed and replenished with conditioned medium containing $60 \%$ LCCM. On day 6 or 7 , adherent cells were harvested using sterile $1 \mathrm{mM}$ $\mathrm{PBS} / \mathrm{EDTA}$ for 15 minutes at $37^{\circ} \mathrm{C}$, followed by a wash with sterile PBS. Cells were analyzed by flow cytometry using antibodies to CD11b and F4/80. Macrophages were identified as $\mathrm{CD} 11 \mathrm{~b}^{+} / \mathrm{F} 4 / 80^{+}$, and purity was routinely between $80-85 \%$.

Preparation of Bone Marrow Derived Dendritic Cell (BMDCs) Culture Medium

The B16 F10 melanoma cell line was obtained from Dr. Cohen (University of Kentucky). The cells were transfected with mrGMCSF to be used as a source of GM-CSF. They were maintained in DMEM containing 10\% heat-inactivated FCS, glutamine $(2 \mathrm{mM})$, penicillin/streptomycin $(100 \mathrm{U} / \mathrm{ml}$ and $100 \mu \mathrm{g} / \mathrm{ml})$, and $2 \mathrm{mg} / \mathrm{ml} \mathrm{G}-418$ to maintain the transfectants. Once the cells were confluent, they were split into flasks with a larger surface area till reach confluency. The cells were split again into larger flasks and grown for 1 week without G418; then they were frozen and used again without G418 for a maximum of 2 weeks of passage. During this period of time, culture supernatant was collected and stored at $-80^{\circ} \mathrm{C}$.

\section{Generation of BMDCs}

Bone marrow cells were harvested from the tibias and femurs of $\mathrm{C} 57 \mathrm{BL} / 6 \mathrm{~J}$, and TLR2/9 DKO mice by flushing with sterile PBS. The RBCs were lysed and the bone marrow cells were washed in media and resuspended in $15 \mathrm{ml}$ of complete conditioned medium. The cell suspension was plated in a $100 \mathrm{~mm}$ petri- dish. On day $3,75 \%$ of the media was removed to get rid of neutrophils and replenished with complete conditioned medium. On day 6-7, non-adherent cells were harvested and dendritic cells were enriched using a mouse Dendritic Cell Enrichment Kit (BD Biosciences; San Jose, CA) following the manufacturer's instructions. The cells were analyzed by flow cytometry using antibodies to CD11b, CD11c, CD80, and CD86. Immature BMDCs were identified as $\mathrm{CD}_{11} \mathrm{~b}^{+} /, \mathrm{CD} 11 \mathrm{c}^{+} / \mathrm{CD} 80^{\mathrm{lo}} / \mathrm{CD} 86^{\mathrm{lo}}$. 


\section{Phagocytosis Assays}

Preparation of Carboxyfluorescein Succinimidyl Ester (CFSE)-Labeled Lyophilized SR for in vitro Phagocytosis Assays

A $5 \mathrm{mg} / \mathrm{ml} \mathrm{SR}$ aliquot was washed with PBS twice by centrifugation at 10,000xg for 5 minutes. The pellet was resuspended in $500 \mu 1$ sterile PBS and CFSE (Invitrogen, Carlsbad, CA) was added at a final concentration of $1 \mu \mathrm{M}$ and incubated at $37^{\circ} \mathrm{C}$ in the dark. The labeled bacteria were washed 3 times with PBS by centrifugation at 10,000xg for 5 minutes. The labeling efficiency was measured by flow cytometry and typically between $80-85 \%$ of the bacteria were labeled.

Preparation of TAMRA Labeled Live SR for in vivo Phagocytosis Assays

An aliquot of $40 \times 10^{8} \mathrm{CFU} / \mathrm{ml}$ live SR was washed with PBS twice by centrifugation at $10,000 \mathrm{xg}$ for 5 minutes. The pellet was resuspended in $400 \mu 1$ sterile PBS then incubated with TAMRA dye (final conc. $12.5 \mu \mathrm{g} / \mathrm{ml}$ ) on ice for $10 \mathrm{~min}$ in the dark. The pellet was washed with PBS 3 times and then resuspended in $1 \mathrm{ml}$ PBS. Labeling efficiency was checked by microscopy, and it was approximately $90 \%$.

In vitro Phagocytosis

In vitro phagocytosis using flow cytometry: $\mathrm{BMDCs}$ or $\mathrm{BMDMs}$ were used for in vitro phagocytosis assays. Ninety $\mu \mathrm{g} / 250 \mu \mathrm{l}$ CFSE labeled SR was opsonized with $8 \%$ normal mouse serum at $37^{\circ} \mathrm{C}$. Following 1 hour incubation, the opsonized bacteria were washed twice with sterile PBS then centrifuged at 10,000xg for 3 minutes. In a $5 \mathrm{ml}$ FACS tube (BD Falcon), $5 \times 10^{5}$ BMDCs were incubated with the opsonized labeled bacteria on ice for 30 minutes to allow the binding of bacteria to the cell surface. Tubes were then incubated at $37^{\circ} \mathrm{C}$ for $20,60,120,180$, or 240 minutes. As a negative control, $10 \mu \mathrm{g} / \mathrm{ml}$ Cytochalasin D (Sigma-Aldrich, St. Louis, MO) was added to inhibit actin depolymerization and hence phagocytosis. Cells were also incubated with unlabeled SR at $37^{\circ} \mathrm{C}$ to gate out fluorescence background and with labeled opsonized SR on ice as another control. Cells were washed twice with cold PBS to stop phagocytosis then $1 \mathrm{ml}$ of $0.4 \%$ Trypan blue was added to quench extracellular fluorescence then cells were washed once with PBS. Finally, the cells were fixed with $100 \mu 14 \%$ paraformaldehyde (BD Cytofix $^{\mathrm{TM}}$ ) for 15 minute on ice. By gating on live cells using Forward and side scatter, the percent of BMDCs that phagocytosed labeled SR was measured by flow cytometry.

In vitro phagocytosis using confocal microscopy: In 24 well plate containing cover slips, WT or TLR2/9 DKO BMDMs $\left(7.5 \times 10^{5}\right.$ cells/well) were cultured in RPMI $5 \%$ FCS for 18 hours to allow adherence. Next day, the cells were incubated with live opsonized TAMRA labeled SR for 1 hour and 2 hours at MOI 50. For Cytochalasin D control, certain wells were treated with Cytochalasin D $(10 \mu \mathrm{M})$ for 30 minutes before incubation with SR. Following incubation times, media were removed and phagocytosis was stopped by cold PBS then wells were washed to remove free SR. The cover slip was removed carefully and placed on a glass slide with mounting media $(50 \%$ glycerol in 
PBS). Each slide contains 3 cover slips representing triplicates from 3 wells. Using LSM 710 confocal microscope, BMDM that contained one or more red dot was counted as one. Cells that contain any dispersed red speckles were not counted as positive cell. Total cells in the field were counted then positive BMDMs that internalize SR were counted; finally, the percentage of BMDMs that phagocytose SR was calculated.

In vivo Phagocytosis

In vivo phagocytosis using flow cytometry: WT and TLR2/9 DKO mice were exposed to $200 \mu \mathrm{g}$ of CFSE labeled lyophilized SR. One hour post-exposure the mice were sacrificed and BALF was collected using $10 \mathrm{ml}$ of sterile $1 \mathrm{mM}$ EDTA-PBS. The cells were recovered from the BALF by centrifugation. The cell suspension was centrifuged for $5 \mathrm{~min}$ at $1500 \mathrm{rpm}$ then extracellular fluorescence was quenched using $1 \mathrm{ml}$ of $0.4 \%$ Trypan blue then incubated for 1 minute at room temp. The cell suspension was washed with PBS then centrifuged for 5 min at 1500rpm. Finally, the cells were fixed using $200 \mu 14 \%$ paraformaldehyde. The percent of alveolar macrophages phagocytosing labeled SR was measured by flow cytometry. Mice exposed to unlabeled SR were used as a negative control to gate out fluorescence background.

In vivo phagocytosis using confocal microscopy: WT and TLR2/9 DKO mice were exposed to $2 \times 10^{8} \mathrm{CFU} / 50 \mathrm{ul}$ TAMRA labeled live SR. One hour post-exposure the mice were sacrificed and BALF was collected using $10 \mathrm{ml}$ of sterile $1 \mathrm{mM}$ EDTA-PBS. The cells were recovered from the BALF by centrifugation and then fixed with $200 \mu 14 \%$ paraformaldehyde. The cell suspension was cytospined using cytospin columns for $5 \mathrm{~min}$. at $1500 \mathrm{rpm}$. The percent of alveolar macrophages phagocytosing labeled SR was counted using LSM 710 Ziess confocal microscope by merging fluorescent field with bright field.

\section{In vitro Stimulation of BMDMs Using SR}

$5 \times 10^{5} \mathrm{BMDMs} /$ well were stimulated with media or $10 \mu \mathrm{g}$ SR. Following 24 hours of stimulation, culture supernatant was harvested for detection of cytokines using Multiplex Sandwich Assay and ELISA.

\section{Multiplex Sandwich Immunoassay}

Cytokines in the culture supernatant harvested from stimulated BMDMs were detected using MILLIPLEX MAP Immunoassay kit (Millipore, Billerica, MA) per manufacturer's instructions. The concentration of the cytokine was determined by setting up standard curves ranging from 3.2 to $10,000 \mathrm{pg} / \mathrm{ml}$. Sample concentrations were extrapolated from four parameter log standard curve in Milliplex software analyst. The whole process of cytokine detection using a luminex instrument was done by Dr. Mark Miller. 


\section{ELISA}

\section{Measurement of IFN $\gamma$}

Mouse IFN $\gamma$ capture antibody (BD Opt EIA, San Diego, CA) was diluted 1:250 in coating buffer (0.1M Sodium Carbonate, $\mathrm{pH} 9.5)$. In a 96 well plate, $100 \mu \mathrm{l}$ of the diluted capture antibody/well was added and incubated at $4{ }^{\circ} \mathrm{C}$. Following an overnight incubation, the plate was washed 5 times with wash buffer (PBS $+0.05 \%$ Tween-20); then the plate was blocked with $200 \mu \mathrm{l}$ assay diluent (PBS $+10 \% \mathrm{FBS}$ )/well and incubated at room temperature. After 1 hour incubation, the plate was washed again. From the stock standard, $2000 \mathrm{pg} / \mathrm{ml}$ was prepared as the highest point in the standard curve; then a 2-fold dilution was prepared from the first concentration, ending with $15.6 \mathrm{pg} / \mathrm{ml}$ as the lowest concentration. One hundred $\mu 1 /$ well from each dilution was plated in duplicates and $100 \mu \mathrm{l} /$ well of the sample was plated as well. The plate was incubated for 2 hours at room temperature with slow rocking, then washed 5 times. Working detector was prepared by diluting both Biotinylated anti-mouse IFN $\gamma$ (BD Opt EIA, San Diego, CA) (1:250) and streptavidin-horseradish peroxidase conjugate (BD Opt EIA, San Diego, CA) (1:250) in assay diluent. One hundred $\mu$ of the working detector/well was added, the plate was sealed, covered, and incubated at room temperature for 30 minutes. The plate was washed 10 times, soaking the wells with washing buffer for 1 minute each time. The plate was developed by adding $100 \mu 1 /$ well TMB substrate (BD Biosciences, San Diego, CA), then incubated for 30 minutes at room temperature in the dark. To stop the color reaction, $50 \mu \mathrm{l} /$ well of $2 \mathrm{~N}$ sulphuric acid was added. The plate was read on a Spectra Max 340 plate reader (Molecular Devices, Sunnyvale, CA) at 450 and 570nm to subtract background absorbance. Data was analyzed by SoftMax ${ }^{\circledR}$ Pro software Version 5 (Molecular Devices, Sunnyvale, CA) using a 4-parameter standard curve.

\section{Measurement of IL-23p19}

Using assay diluent, highest standard concentration $(1000 \mathrm{pg} / \mathrm{ml})$ was prepared from the standard stock; then a 2-fold dilution was prepared from the first concentration, ending with $7.8 \mathrm{pg} / \mathrm{ml}$ as the lowest concentration. One hundred $\mu \mathrm{l} /$ well from each dilution was plated in duplicate, and $100 \mu \mathrm{l} /$ well of the sample was plated as well into a 96-well plate precoated with anti-mouse IL-23p19. Following 2 hours of incubation at room temperature, the plate was washed with assay diluent 5 times. Biotinylated antimouse IL-12/23p40 (eBiosciences, San Diego, CA) was diluted 1:250 in assay diluent. $100 \mu \mathrm{l} /$ well was plated and incubated at room temperature for 1 hour, then washed for 5 times. 100ul of 1:250 diluted streptavidin-horseradish peroxidase conjugate (eBiosciences, San Diego, CA) was added and incubated in the dark for 30 minutes. After washing for 7 times with assay diluent, $100 \mu 1 /$ well TMB substrate (eBiosciences, San Diego, CA) was added; then the plate was incubated for 15 minutes at room temperature in the dark. To stop the color reaction, $50 \mu \mathrm{l} /$ well of $2 \mathrm{~N}$ Sulphuric acid was added. The plate was read on a Spectra Max 340 plate reader (Molecular Devices, 
Sunnyvale, CA) at 450 and $570 \mathrm{~nm}$ to subtract background absorbance. Data was analyzed by SoftMax pro software Version 5 using a 4-parameter standard curve.

\section{Statistical Analysis}

Values were expressed as mean \pm S.D. For comparison of means between two populations of unpaired data, unpaired student's t-test was used. For comparison between more than two groups, One-way ANOVA followed by Tukey's post ANOVA test was used. GraphPad Prism 5 was used to perform all tests. Differences were considered significant at $\mathrm{P}<0.05$.

\section{RESULTS}

\section{TLRs 2 and 9 Did Not Affect Phagocytosis Ability of BMDCs}

One explanation for the decrease in Th17 cells in the lung is deficient phagocytosis of SR by TLR2/9 deficient APCs. To test this hypothesis, we generated BMDCs in vitro from WT and TLR2/9 DKO mice then incubated them with CFSE labeled SR at different time points and the internalization of CFSE labeled SR was measured by flow cytometry. The results revealed that there was no significant difference in the phagocytosis ability of either WT or TLR2/9 DKO at any time points; both WT and DKO were able to internalize labeled SR efficiently (Fig. 4-2). We used cytochalasin $\mathrm{D}$ as a control since it inhibits phagocytosis and we did find an inhibition of phagocytosis using cytochalasin D. We also used serum from C57BL/6J mice exposed to SR for 2 weeks to develop antibodies against SR and use the serum for more efficient opsonization which will result in more efficient phagocytosis; however, we didn't find a significant difference in phagocytosis using immunized or non-immunized serum. In conclusion, these results demonstrated that TLR2 and 9 did not affect the phagocytosis ability of BMDCs.

\section{TLRs 2 and 9 Did Not Affect Phagocytosis Ability of BMDMs}

We next analyzed events that occur downstream from the polarization of naïve $\mathrm{T}$ cells. Once effector $\mathrm{T}$ cells exit lymph nodes after their differentiation, they migrate to target tissue to perform their effector function (in our model the target tissue is the lung). Effector $\mathrm{T}$ cells require antigen presentation in the target tissue and the candidate cells in our model were interstitial macrophages.

We hypothesized that interstitial macrophages in TLR2/9 DKO mice were not efficient in phagocytosis and hence deficient in their antigen presentation ability to stimulate effector Th17 cells in the lungs. To test this hypothesis, we tried to isolate interstitial macrophages from WT and TLR2/9 DKO mice but we faced several 


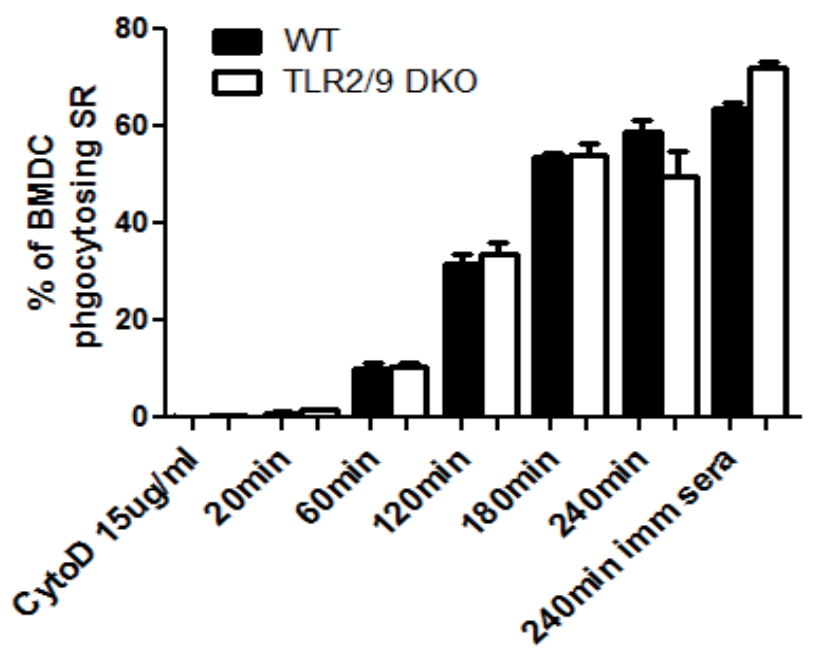

Figure 4-2. Detection of internalized CFSE labeled SR in BMDCs using flow cytometry.

One hundred thousand BMDCs were incubated with CFSE labeled SR $(360 \mu \mathrm{g} / \mathrm{ml})$ at different time points. The percentage of BMDCs phagocytosing labeled SR was determined using flow cytometry. Each column represents $n=3$ except 20 min time point was $n=2$. Results were expressed as means $\pm \mathrm{SD}$. Unpaired student's t-test was used to compare between WT and TLR2/9 DKO phagocytosis ability within each time point. 
limitations in the isolation technique such as the requirement to pool large number of mice and low purity. We decided to generate macrophages from bone marrow in vitro which gave us high yield and purity; therefore, we used BMDMs in phagocytosis assays.

In vitro Phagocytosis Using Flow Cytometry

We designed an in vitro phagocytosis assay using opsonized CFSE labeled SR to incubate with WT or TLR2/9 DKO BMDMs at different time points. The efficiency of SR internalization in both WT and TLR2/9 DKO BMDMs was measured by flow cytometry and then confirmed by confocal microscopy. We started with in vitro phagocytosis using flow cytometry to determine the best time point to be used next in the confocal microscopy. As shown in Fig. 4-3, there was not a significant difference at any time point

\section{In vitro Phagocytosis Using Confocal Microscopy}

The phagocytosis results using flow cytometry revealed that both TLR2 and 9 did not affect SR uptake ability by macrophages; however, these results need to be confirmed using a different technique. We used confocal microscopy to test if our flow cytometry results will be confirmed or not. WT or TLR2/9 DKO BMDMs were incubated with opsonized TAMRA labeled live SR at MOI 50 for one and two hours. Cytochalasin D was used as a control to inhibit phagocytosis. The internalization of SR was visualized by confocal microscopy after merging bright with fluorescent field. The phagocytosed labeled SR was visualized as red dot inside the cell. As shown in Fig. 4-4, there was no significant difference in phagocytosis ability of WT and TLR2/9 DKO.

\section{TLRs 2 and 9 Did Not Affect Phagocytosis Ability of AMs}

The in vitro phagocytosis results from both confocal and flow cytometry confirmed each other. To confirm the in vitro phagocytosis experiments, we performed in vivo phagocytosis using AMs. Mice were exposed intranasally to labeled SR. One hour later, BAL was performed to collect BAL cells. The phagocytosis ability of WT and TLR2/9 DKO was determined by flow cytometry and confocal microscopy.

In vivo Phagocytosis Using Flow Cytometry

In vivo phagocytosis using $\mathrm{AMs}$ was done to confirm in vitro phagocytosis results. WT or TLR2/9 DKO mice were exposed to CFSE labeled SR. One hour later BAL was performed to collect BAL cells. The uptake of SR by AMs was measured by flow cytometry. As shown in Fig. 4-5, both WT and TLR2/9 DKO AMs were able to phagocytose CFSE labeled SR. 


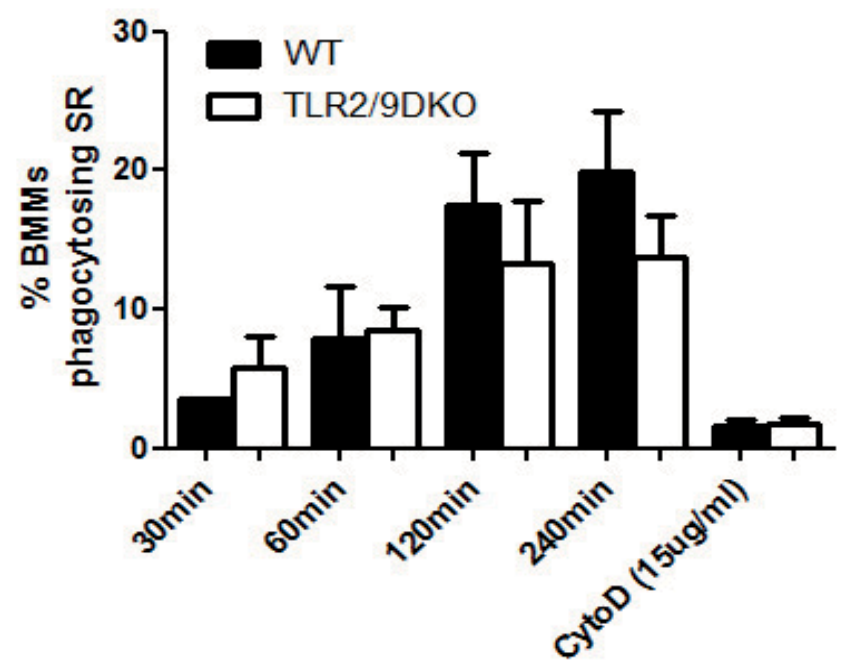

Figure 4-3. Detection of internalized CFSE labeled SR in BMDMs using flow cytometry.

In a 96 well plate, $5 \times 10^{5}$ BMDMs were incubated with CFSE labeled SR $(360 \mu \mathrm{g} / \mathrm{ml})$ at different time points. The percentage of BMDMs phagocytosing labeled SR was determined using flow cytometry. Each column represents $n=2$. Results were expressed as means \pm SD. Unpaired student's t-test was used to compare between WT and TLR2/9 DKO phagocytosis ability within each time point. 
Figure 4-4. Detection of internalized TAMRA labeled SR in BMDMs using confocal microscopy.

Panels (A-P) represent confocal images by merging bright field with fluorescence field. Panels A and C are representative fields for WT and TLR2/9 DKO BMDMs phagocytosing TAMRA labeled SR (MOI 501 hour) respectively. Panels B and D are cropped image of the same fields. Panels E-H are same conditions as above except cytochalasin D was added. Panels I and K are representative fields for WT and TLR2/9 DKO BMDMs phagocytosing TAMRA labeled SR (MOI 502 hours) respectively. Panels J and L are cropped image of the same fields. Panels M-P represents the same conditions except cytochalasin D was added. Data are representative of 12-15 fields/sample for each condition. (Q) Quantification of phgaocytosis. Each column represents observation from 9 fields (3 fields/well). Total cells/field was counted then cells phagocytosing SR were counted to calculate percentage. Results were represented as means \pm SD. Using unpaired student's test, means between groups incubated with SR and group treated with Cytochalain $\mathrm{D}$ were compared. ( $* * * \mathrm{P} \leq 0.0001$ significant difference from cytochalasin D control.) 

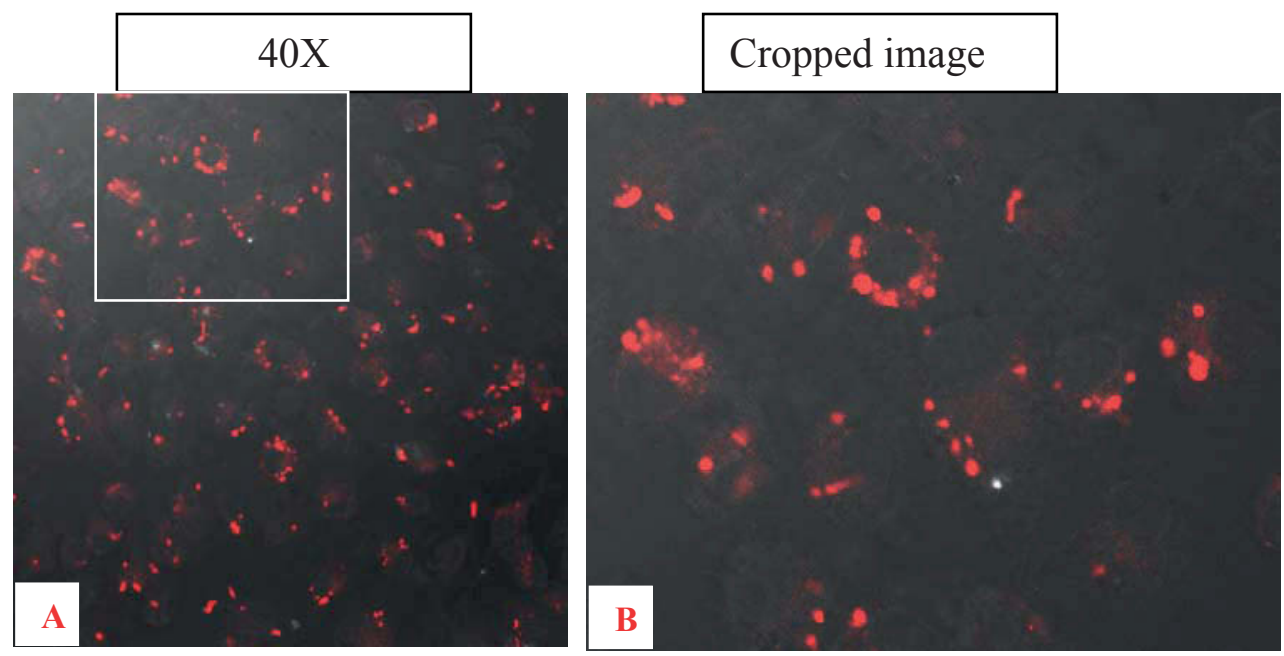

\begin{tabular}{|c|}
\hline WT \\
MOI 50 \\
$1 \mathrm{hr}$ \\
\hline
\end{tabular}
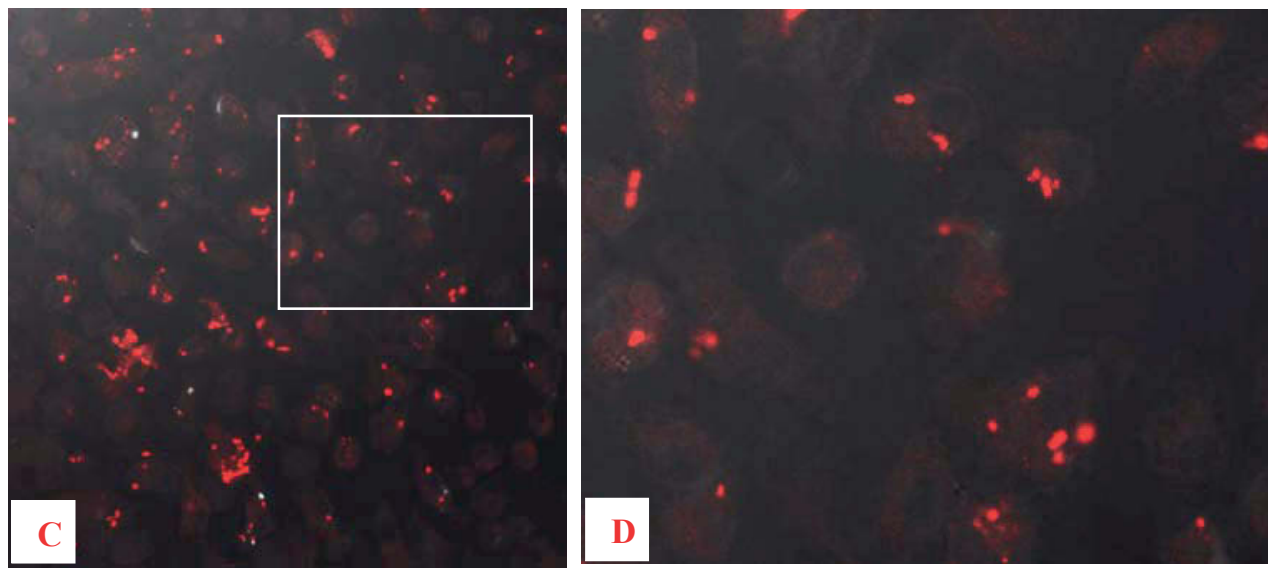

TLR2/9

DKO

MOI 50

$1 \mathrm{hr}$ 

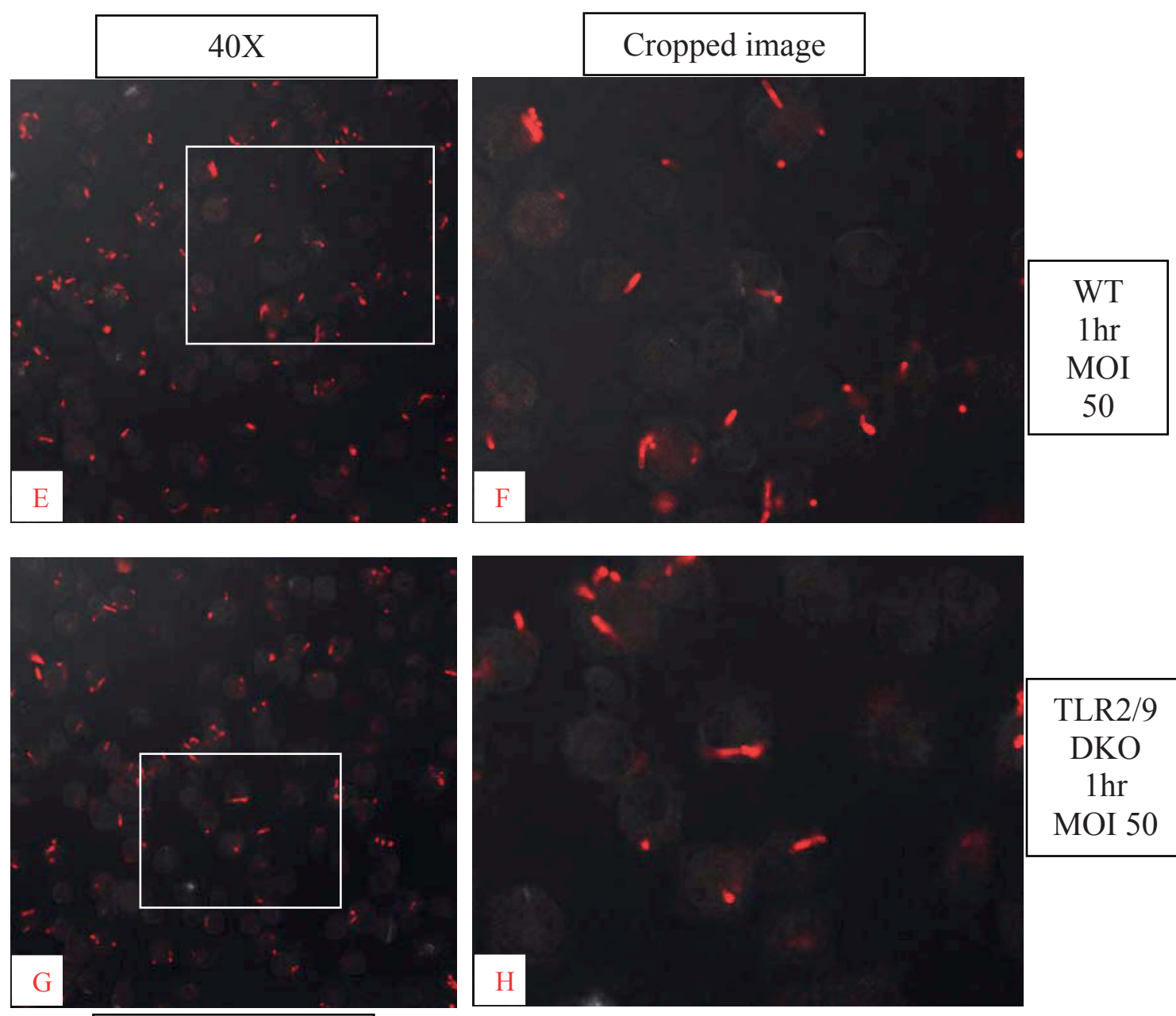

Cytochalasin D

Figure 4-4. (Continued). 

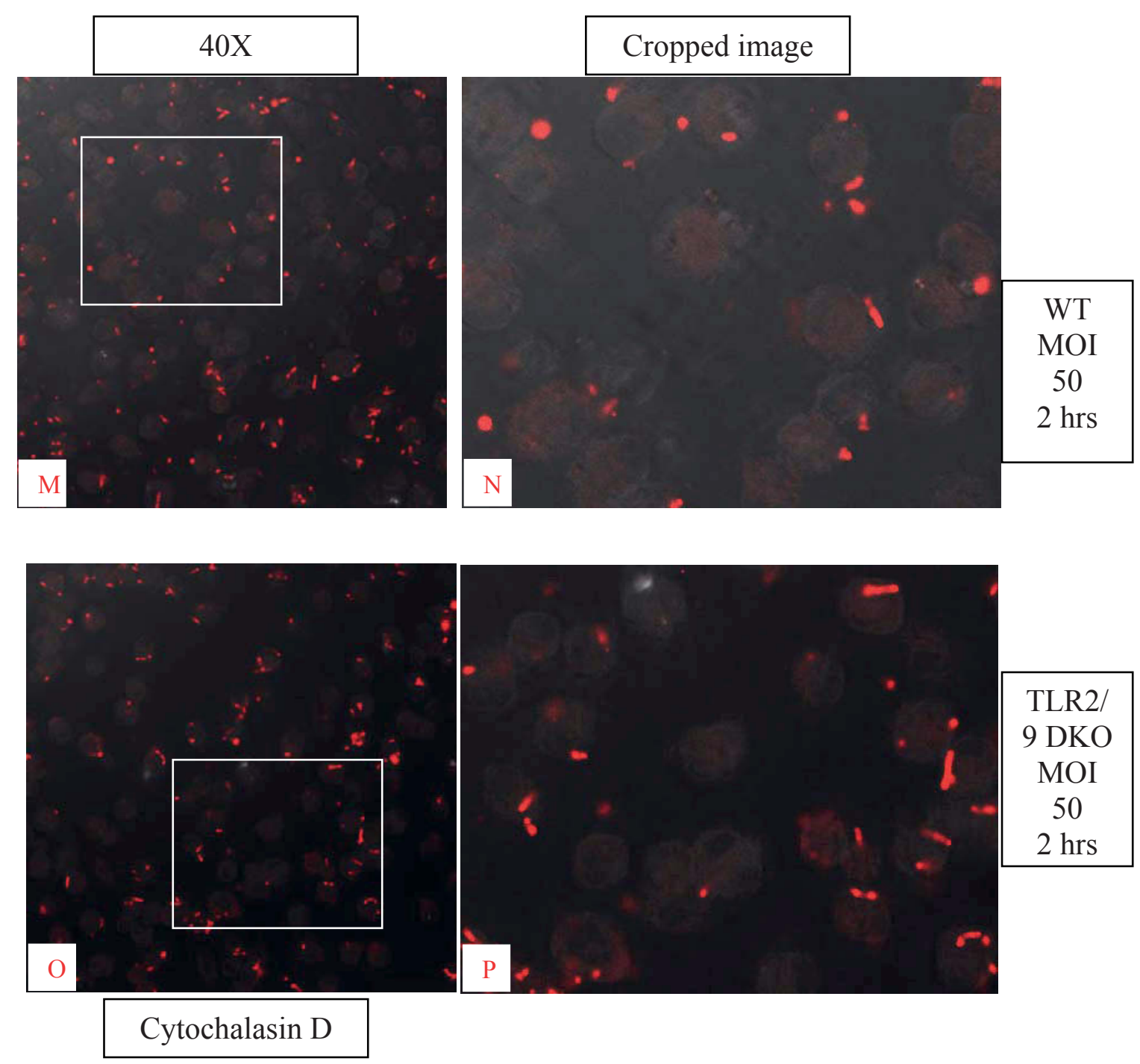

Figure 4-4. (Continued). 


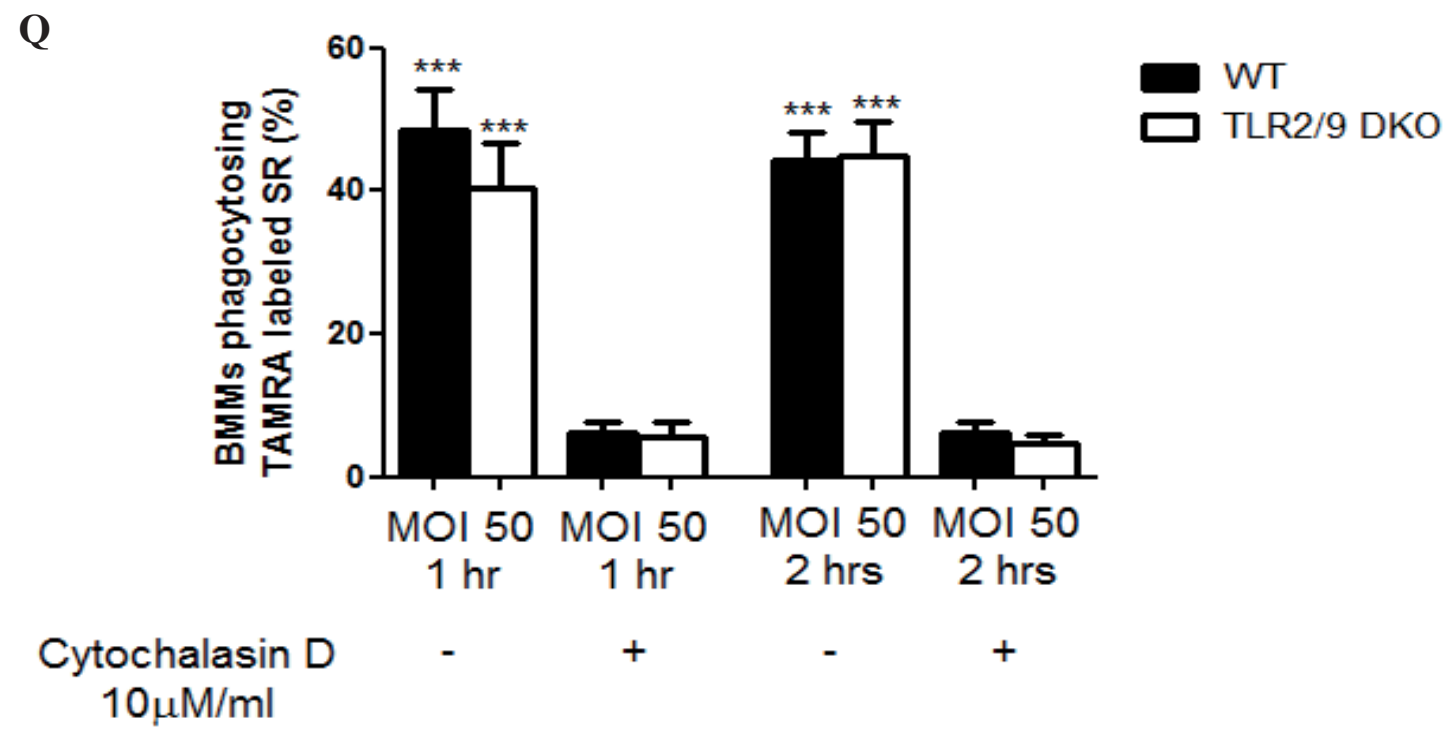

Figure 4-4. (Continued). 


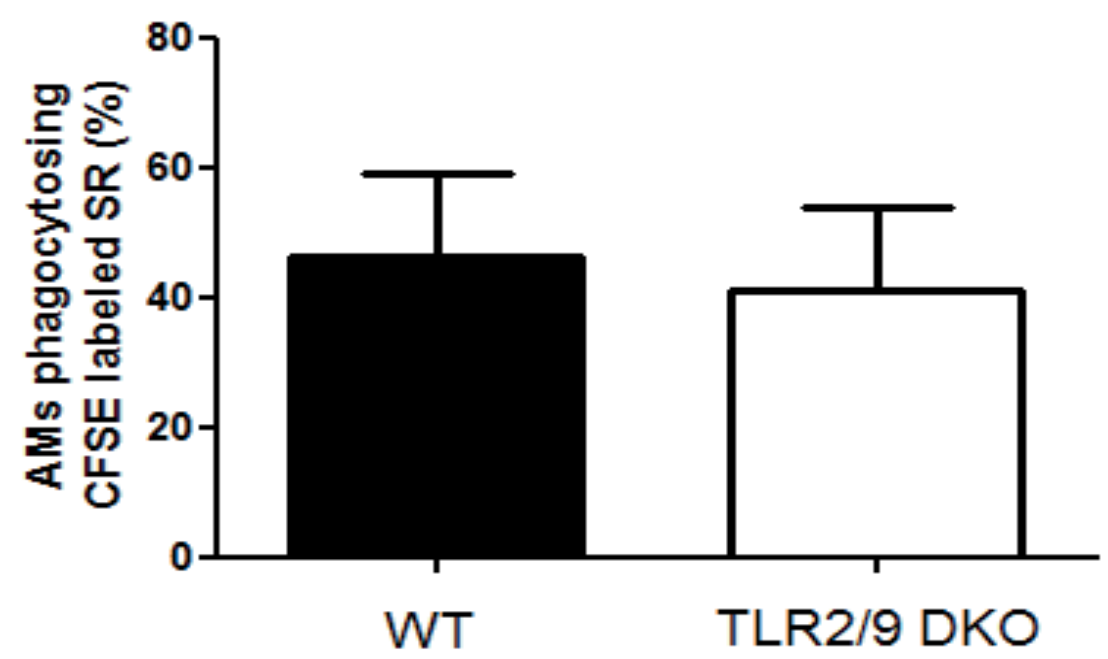

Figure 4-5. Detection of internalized CFSE labeled SR in AMs using flow cytometry.

WT or TLR2/9 DKO mice were exposed to $200 \mu \mathrm{g}$ of CFSE labeled SR. One hour later mice were sacrificed and BAL was performed to collect BAL cells. Extracellular fluorescence was quenched by Trypan Blue, and then washed with PBS. The cells were fixed with $4 \%$ paraformaldhyde, and finally the data was acquired on the flow cytometer. Each column represents $n=8-9$ combined from 5 independent experiments. Results were expressed as means $\pm \mathrm{SD}$. Unpaired student's t-test was used to compare mean between WT and TLR2/9 DKO phagocytosis percentage. 
In vivo Phagocytosis Using Confocal Microscopy

To confirm the phagocytosis results from the flow cytometry method, we used confocal microscopy to examine if flow cytometry results match the confocal results. Both WT and TLR2/9 DKO were exposed to $2 \times 10^{8} \mathrm{CFU} / 50 \mu \mathrm{l}$ TAMRA labeled live SR. One hour later, BAL was performed to collect BAL cells. The BAL cells underwent cytospin on a glass slide. AMs phagocytosing labeled SR were visualized using confocal microscopy. As shown in the Fig. 4-6, there was no significant difference in the phagocytosis ability of both WT and TLR2/9 DKO AMs.

\section{TLRs 2 and 9 Did Not Affect the Antigen Presentation Ability of Splenic APCs}

Following $\mathrm{T}$ cell differentiation in the lymph nodes, effector $\mathrm{T}$ cells migrate to their target tissues, the site of inflammation; however, they still require antigen presentation by APCs to maintain their effector function there in. The candidate APCs in the lung parenchyma are lung interstitial macrophages. Since we observed a decrease in the frequency of Th17 cells in the lungs of TLR2/9 DKO mice compared to WT counterparts, we sought to examine the efficiency of WT and TLR2/9 DKO APCs in stimulating effector Th17 cells to produce IL-17 as effector cytokine.

Phagocytosis is a process that precedes antigen presentation. We already demonstrated in the previous sections that TLRs 2 and 9 do not affect SR uptake by macrophages and dendritic cells. Consequently, we examined antigen presentation efficiency of macrophages using antigen presentation assay.

In this assay, we tested the ability of both WT and TLR2/9 DKO APCs to present SR antigen to WT or TLR2/9 DKO effector Th17 cells isolated from lungs; and also if this stimulation of Th17 cells would produce IL-17. The measurement of Th17 effector function e.g. production of IL-17 is a reflection of the antigen presentation efficiency of APCs. The assay was set up as described in the methods section.

The results demonstrated that both WT and TLR2/9 DKO CD4 $4^{+} \mathrm{T}$ cells were able to produce IL-17 regardless of the phenotype of APCs (WT or TLR2/9 DKO), indicating that TLR2/9 DKO APCs were equally efficient to present SR antigen to WT or TLR2/9 DKO CD4 ${ }^{+} \mathrm{T}$ cells. In conclusion, these results demonstrated that both WT and TLR2/9 DKO APCs were equally efficient in SR presentation to WT or TLR2/9 DKO $\mathrm{CD} 4^{+} \mathrm{T}$ cells (Fig. 4-7A).

We also used SR lysate as a source of SR soluble antigen to examine if TLRs 2 and 9 affect other internalization pathways than phagocytosis. We cocultured WT or TLR2/9 DKO CD4 ${ }^{+}$T cells with WT or TLR2/9 DKO APCs in the presence or absence of SR lysate. We found that both were able to present SR antigen and hence stimulate Th17 cells to produce IL-17 as effector cytokine (Fig. 4-7B). 
Figure 4-6. Detection of internalized TAMRA labeled SR in AMs using confocal microscopy.

WT or TLR2/9 DKO mice $(\mathrm{n}=3)$ were exposed to $2 \times 10^{8} \mathrm{CFU} / 50 \mu 1$ TAMRA labeled live SR. One hour later mice were sacrificed and BAL was performed to collect BAL cells. The cells underwent cytospin on a glass slide. Panels (A-D) represent confocal images by merging bright with fluorescent fields. A and B are WT images while C and D TLR2/9 DKO images. (E) Quantification for 24 fields from 3 mice. Results were expressed as means \pm SD. Unpaired student's t-test was used to compare between WT and TLR2/9 DKO phagocytosis ability. 

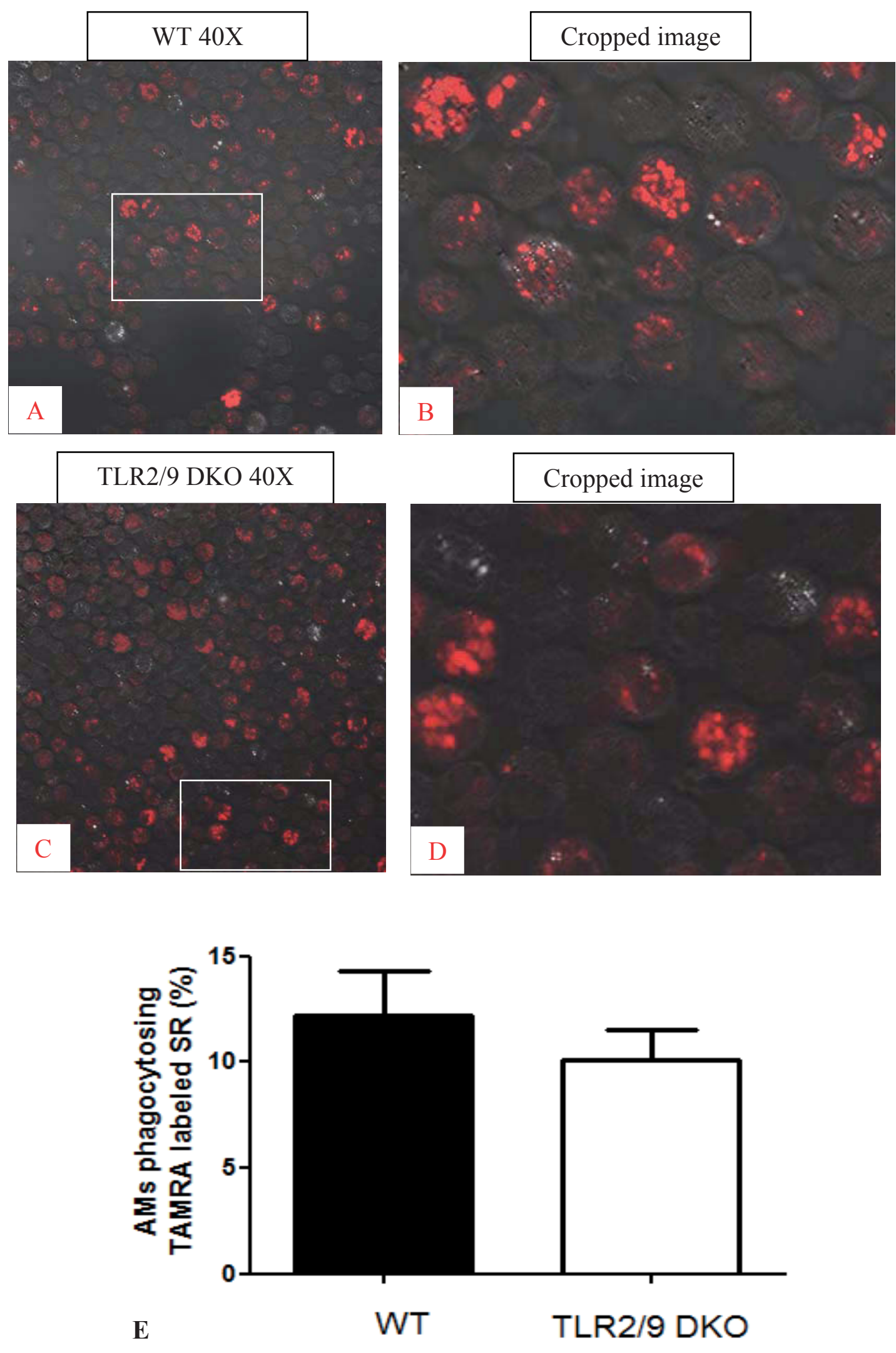
(A)

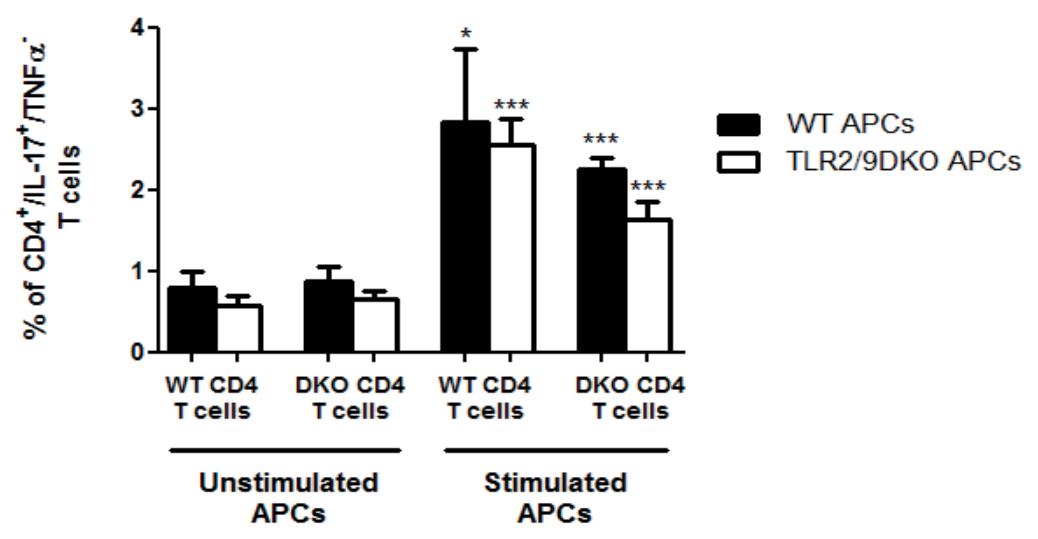

(B)

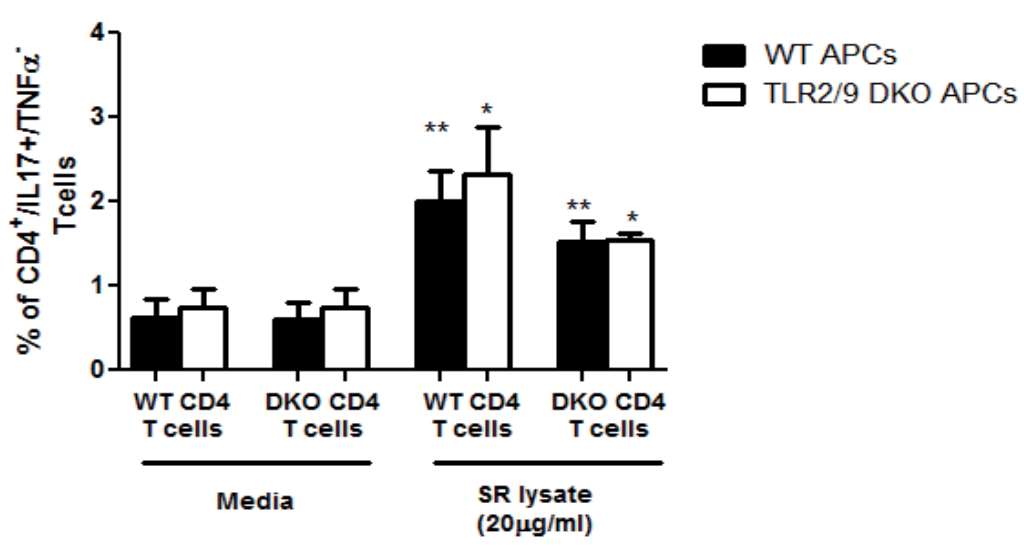

Figure 4-7. TLRs 2 and 9 did not affect antigen presentation ability of splenic APCs.

WT and TLR2/9 DKO mice were exposed to SR (150ug) for 3 wks then CD4 ${ }^{+} \mathrm{T}^{-}$cells were isolated with a negative selection kit (BD IMag) from lungs. (A) WT or TLR2/9 DKO CD4 ${ }^{+} \mathrm{T}$ cells were incubated with HKSR stimulated WT or TLR2/9 DKO APCs for 18 hours. As negative control, unstimulated WT or TLR2/9 DKO APCs were incubated with WT or TLR2/9DKO CD4 ${ }^{+} \mathrm{T}$ cells. Following cell surface staining using anti-CD4 and anti-TCR $\beta$ antibodies, intracellular cytokine staining for IL-17 and TNF $\alpha$ was performed. Each bar represent $n=4-6$. Results were expressed as means $\pm \mathrm{SD}$ $* * * \mathrm{P} \leq 0.0001$ and $* \mathrm{P}<0.02$ which is significant difference from the unstimulated control using unpaired student's test. (B) WT or TLR2/9 DKO CD4 ${ }^{+} \mathrm{T}$ cells were incubated with WT or TLR2/9 DKO APCs in the presence or absence of SR lysate for 8 hours.

Following cell surface staining using anti-CD4 and anti-TCR $\beta$ antibodies, intracellular cytokine staining for IL-17 and TNF $\alpha$ was performed. Each bar represents $n=2-3$. Results were expressed as means $\pm \mathrm{SD} * \mathrm{P} \leq 0.05$ and $* * \mathrm{P}<0.01$ which is a significant difference from media control using unpaired student's test. 


\section{Effect of TLRs 2 and 9 on Cytokine Expression from BMDMs}

We hypothesized that cytokines produced by interstitial macrophages play a role in regulation of the inflammatory environment in the lungs and affect effector function of the T cells. In order to test this hypothesis, we stimulated BMDMs from WT, TLR2/9 DKO, and MyD88 KO in vitro with lyophilized SR for 24 hours then we collected the culture supernatant and measured IL-6, IL-10, IL-12p70, and TNF $\alpha$ using Multiplex sandwich immunoassay. We also measured IL-23 and IFN $\gamma$ using ELISA. We found a significant down regulation in the expression of the proinflammatory cytokines IL-6 and TNF $\alpha$ as well as the anti-inflammatory cytokine IL-10 from TLR2/9 DKO and MyD88 KO BMDMs compared to WT stimulated BMDMs. We did not find a significant difference between WT and TLR2/9 DKO when we measured IL-12p70 however; there was a significant down regulation in MyD88 KO BMDMs. We didn't detect IL-23 and IFN $\gamma$ using ELISA. In conclusion, TLR2 and 9 and MyD88 down regulate IL-6, IL-10, IL12p70, and TNF $\alpha$ expression (Fig. 4-8).

\section{DISCUSSION}

\section{TLRs 2 and 9 Did Not Affect the Phagocytosis Ability of DMDMs and BMDCs}

The process of naïve $\mathrm{T}$ cell differentiation to effector $\mathrm{CD} 4^{+} \mathrm{T}$ cell is crucial for initiation of adaptive $\mathrm{T}$ cell immunity. It requires a combination of sequential processes take place at different anatomical sites. The first process occurs at the site of infection where immature DCs, that have a high phagocytic activity, phagocytose the microbial pathogen. Consequently, DCs undergo a maturation program allowing them to migrate to the regional lymph nodes and present the microbial antigen to naïve $\mathrm{T}$ cells. Therefore, phagocytosis is an indispensable process for antigen presentation.

Previous results from our lab have shown that following three weeks of SR exposure, the frequency of Th17 cells in the lungs of TLR2/9 DKO mice was significantly less compared to WT counterparts. Several possibilities underlie this observation. One possibility could be deficiency in the capability of TLR2/9 DKO immature DCs in the lungs to phagocytose the bacteria; hence, there will be a defect in antigen presentation that will result in impairment in polarization of naïve $\mathrm{T}$ cells to effector Th17. To test this hypothesis, we first measured the phagocytosis ability of WT and TLR2/9 DKO BMDCs using CFSE labeled SR. The results revealed that there was not a significant difference in the phagocytosis capability for either WT or TLR2/9 DKO BMDCs at different time points. This data suggested that both WT and TLR2/9 DKO BMDCs were equally efficient in phagocytosis.

Next, we planned to measure costimulatory molecules, MHCII, and cytokine expression using BMDCs. However, we faced several limitations regarding the purity of BMDCs. We only obtained $40-50 \% \mathrm{CD}_{11 \mathrm{c}^{+}}$as a marker for DCs. Consequently, we 

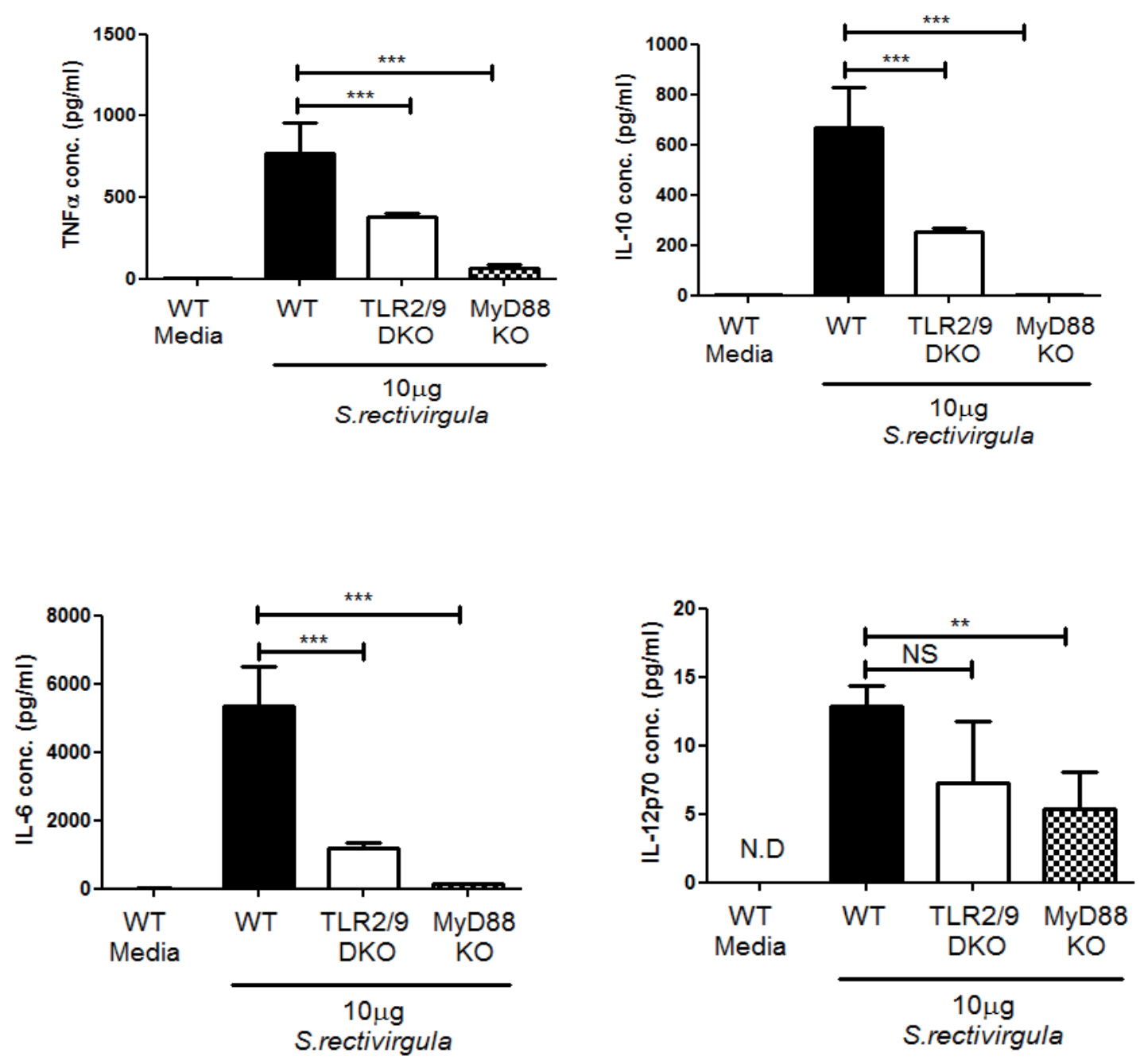

Figure 4-8. Expression of IL-6, TNF $\alpha$, IL-10, and IL-12p70 from WT, TLRs 2/9 DKO and MyD88 KO BMDMs following in vitro SR stimulation.

In a 96 well plate, $5 \times 10^{5}$ cells/well were plated and stimulated with media or $10 \mu \mathrm{g}$ SR. Twenty-four hours later culture supernatant was collected to measure the cytokines using Multilex sandwich assay. Each bar represents $n=4$. Results were represented as means \pm SD. One way ANOVA followed by Tukey's post ANOVA test was used to compare the means of three groups $(* * * \mathrm{P}<0.0001)$. N.D (not detected). 
shifted our efforts to optimize a protocol for isolation of mouse splenic DCs. We obtained $\sim 65 \%$ CD $11 \mathrm{c}^{+}$cells but the yield was very low $\left(2-3 \times 10^{5}\right.$ cells from 2 spleens $)$. As an alternative way to circumvent these obstacles, we might use mediastinal lymph nodes (draining lymph node for the lungs) from three weeks SR exposed mice to check expression of costimulatory molecules and cytokines using intracellular cytokine staining by gating on dendritic cells. In order to compare results to unexposed mice, we need to pool a large number of unexposed mice.

As discussed earlier, the defect in factors required for polarization of naïve $\mathrm{T}$ cells by DCs was only one possibility that lies behind the decrease of Th17 frequency in the lungs of TLR2/9DKO mice. The other possible rational could be a defect in the antigen presentation capability of lung interstitial macrophages to present SR antigen to effector $\mathrm{T}$ cells. Following polarization of naïve T cells in the lymph nodes, effector T cells migrate to the site of infection to start their effector function however; they still require antigen presentation and costimulatory molecules provided by interstital macrophages at the site of infection. To test if there is a defect in TLR2/9 DKO APCs' antigen presentation ability, first we examined their phagocytosis ability as a process that precedes antigen presentation. We started our initial studies by setting up in vitro phagocytosis assays using BMDMs. We tried isolating interstitial macrophages from WT and TLR2/9 DKO but one of the main obstacles was pooling large number of mice. For that reason, we used BMDMs in the in vitro phagocytosis assays. We found that both WT and TLR2/9 DKO BMDMs were equally efficient in SR uptake using both flow cytometry and confocal microscopy techniques. To confirm the in vitro data, we setup in vivo phagocytosis assays using flow cytometry and confocal microscopy techniques. Our data demonstrated that WT and TLR2/9 DKO AMs were able to phagocytose SR efficiently; however, the results of in vivo phagocytosis obtained from flow cytometry were higher than results obtained from confocal microscopy. There are several reasons that could explain this difference. One explanation could be a matter of technique sensitivity, since in the confocal microscopy when we analyzed the data; we set up certain criteria when counting the cells phagocytosing SR. Only cells with red dots were counted and any red dispersed speckles were not counted which could be detected by flow cytometry as a positive signal.

One of the drawbacks of using confocal images by merging fluorescence with the bright field is proving whether bacterium is intracellular or attached to the cell. We can tell that labeled SR is inside by looking for the borders of the cell which includes the cytoplasm and the nucleus; however, SR could adhere to the cell surface and will not be washed away. Finally, we do not know in which compartment the bacterium resides. There are a couple ways to circumvent these pitfalls. First, we could label the bacteria with Oregon green followed by biotin labeling then incubate the biotinylated labeled bacteria with the cells to allow phagocytosis (1 or 2 hours). Following incubation, rhodamine-sterptavidin would be added for 30 minutes. If the biotinylated labeled bacterium is phagocytosed, it will be visualized in the FITC (green) channel only, and if the bacterium is extracellular or attached to cell surface, it will be visualized in both channels FITC and rhodamine and appear as yellow on merging between the two channels. In other words, extracellular or adhered bacterium will be yellow while 
intracellular bacterium will be green on merging between 2 channels. Using lysomal markers (LAMP1 and LAMP2) could be another way to confirm the internalization of the bacterium. Both labeled bacterium and cells labeled with lysosomal markers are detected in different channels. Only internalized bacterium will be visualized by merging between the two channels.

\section{TLRs 2 and 9 Did Not Affect the Antigen Presentation Ability of Splenic APCs}

Following phagocytosis, the antigen is processed to be presented by MHC. We already tested the phagocytosis ability of both WT and TLR2/9 DKO BMDMs and BMDCs. As a subsequent process, we will investigate antigen presentation process to examine if there is a difference in the antigen presentation ability of both WT and TLR2/9 DKO APCs. If we observe a difference, we will start investigating the steps that precedes antigen presentation i.e. antigen processing that might lead to a defect in antigen presentation. A defect in antigen presentation might reflect why we see a decrease in the frequency of Th17 cells in lungs of TLR2/9 DKO mice.

We hypothesized that TLR2/9 DKO APCs were not able to phagocytose and hence present SR antigen to effector Th17 cells in the lungs compared to WT APCs that can phagocytose and present SR antigen to effector T cells. To test this hypothesis, we already did in vitro and in vivo phagocytosis assays and our results demonstrated that both WT and TLR2/9 DKO macrophages and dendritic cells were able to phagocytose SR efficiently. Next we used an antigen presentation assay to examine the ability of both WT and TLR2/9 DKO APCs to present SR antigen to effector T cells. We used both HKSR and SR lysate in the antigen presentation assays. Using HKSR, we measured IL-17 production from $\mathrm{CD}^{+} \mathrm{T}$ cells to test their effector function. If APCs were not able to present SR to T cells, we will find low percentage of Th17 cells. In order to confirm the production of IL-17 is actually from antigen specific T cells, one control should be added to the experiment: coculturing naïve $\mathrm{CD}^{+} \mathrm{T}$ cells with APCs presenting SR antigen. Since naïve T cells are not antigen specific to SR, we expected to find no response from naive $\mathrm{T}$ cells.

We also used SR lysate to check if TLRs2 and 9 affect other pathways other than phagocytosis that soluble antigens use to be internalized. Both APCs and T cells were cocultured together in the presence or absence of SR lysate then IL-17 production was measured from $\mathrm{CD}^{+} \mathrm{T}$ cells using intracellular cytokine staining. Since there is a possibility that PAMPs in the SR lysate can stimulate $\mathrm{T}$ cells directly to produce cytokines, one control should be added to the experiment: stimulation of $\mathrm{CD} 4^{+} \mathrm{T}$ cells isolated from lungs of mice exposed to SR for three weeks with SR lysate then determine the production of IL-17 from $\mathrm{CD} 4{ }^{+} \mathrm{T}$ cells with intracellular cytokine staining. In both cases (using HKSR or SR lysate), the results demonstrated that both WT and TLR2/9 DKO APCs were able to present SR antigen efficiently to effector $\mathrm{CD}^{+} \mathrm{T}$ cells stimulating them to produce IL-17as effector cytokine for Th17 cells. However, TLR2/9 DKO T cells tend to produce less IL-17 than WT CD4 T cells which requires 
further investigation. One experiment would be stimulation of WT or TLR2/9 DKO $\mathrm{CD} 4^{+} \mathrm{T}$ cells with anti-CD3 then measure production of IL-17 by intracellular staining.

Consistently, we observe $\sim 3-4 \%$ Th17 cells following antigen presentation assays; however, Th17 response following three weeks of SR exposure was $\sim 15 \%$ in WT mice as shown in Chapter 2 Fig. 2-7. The discrepancy in the results could be attributed to differences in experimental setups. We determined Th17 response in the lungs of WT mice by using total lung cells then stimulated the cells with SR lysate. The total lung cells already had effector T cells with APCs e.g interstitial macrophages as an example of efficient antigen presenting cell. On the other hand, in the antigen presentation assay, we tried to mimic the in vivo environment by incubating splenic APCs with HKSR to phagocytose, process, and present SR antigen then coculture them with T cells isolated from lungs. The splenic APCs could be less efficient in antigen presentation since they are not pure macrophages only. They are contaminated with other cells e.g. B cells which are less efficient antigen presenting cells. Another possible explanation is the ratio of APCs to T cells. We used a ratio of 1:1 in the antigen presentation assay. On the other side, the in vivo situation was different since the ratio of T cells to APCs might be lower than 1:1(i.e. 1:5, 1:10 or less). In one study, it was determined that the number of antigen$\mathrm{MHC}$ complexes required to stimulate effector $\mathrm{T}$ cells vs. naïve $\mathrm{T}$ cells. It was found that effector T cells require $\sim 40$ antigen-MHC complexes while naïve T cells require $\sim 400$ antigen-MHC complexes [214]. To conclude, our studies demonstrated that both WT and TLR2/9 DKO BMDCs and BMDMs were equally efficient in phagocytosis of labeled SR. Furthermore, WT and TLR2/9 DKO splenic APCs were capable of SR antigen presentation and hence stimulation of the Th17 effector function (production of IL-17 from Th17 cells).

These studies led us to think about different alternatives to explain the original observation that following three weeks of SR exposure, TLR2/9 DKO mice were characterized by fewer Th17 cells in their lungs compared to their WT counterparts. DCs are crucial in initiating adaptive immune responses by polarizing naïve $T$ cells in the lymph nodes. Our studies tested the phagocytosis ability of BMDCs, and we found that both WT and TLR2/9 DKO BMDCs were equally efficient in up taking labeled SR. We tried to examine expression of costimulatory molecules and cytokine expression from BMDCs; however, we faced a number of limitations as mentioned before. We hypothesized that TLR2/9 DKO BMDCs produce less IL-6 and TGF $\beta$ (cytokines required for Th17 differentiation); consequently, they affect differentiation of naïve $\mathrm{T}$ cells to Th17 cells. Another possibility is that differentiation of Th17 cells is equally efficient in both WT and TLR2/9 DKO lymph nodes, but there is a defect in the chemokines required for migration of effector Th17 cells from lymph node to the lung.

\section{TLRs 2 and 9 and MyD88 Affect Cytokine Expression by BMDMs}

As mentioned before in chapter one, the cross-talk between the cells within the granuloma regulate the microenvironment. The cytokines produced by macrophages in the lungs might play a role in regulating effector $\mathrm{T}$ cells as well as the cytokine 
environment in the granuloma. Following in vitro SR stimulation, we found a significant decrease in IL-6 production from TLR2/9 DKO and MyD88 KO BMDMs compared to WT BMDMs. IL-6 is a proinflammatory cytokine and it is required for Th17 differentiation. IL-6 can also regulate T cell proliferation [215]. IL-6 KO ova specific T cells were not able to proliferate [215]. Since we already showed that both WT and TLR2/9 DKO BMDMs were equally efficient in phagocytosis and antigen presentation, we could hypothesize that the deficiency of IL-6 in the lungs of TLR2/9 DKO might affect proliferation of SR antigen specific Th17 cells; however, further experiments need to be done before making this conclusion. We hypothesized that expression of IL-10 from BMDMs will affect the expression of IL-17 expression from Th17. It has been shown that IL-10 down regulates IL-17 expression from $\mathrm{CD}^{+} \mathrm{T}$ cells [216]; however, we found a downregulation in IL-10 from TLR2/9 DKO BMDMs. IL-10 could be expressed by other cell types in the lungs. Both IL-12p70 and IFN $\gamma$ cytokines could play a role in the conversion of Th17 to Th1 cells [171]. Therefore, we might suggest that both cytokines are responsible for decrease in the Th17 effector cells in the lungs of TLR2/9 DKO mice. IL-23 is a survival factor for Th17 cells and we didn't detect it from BMDMs. It might be another cell population is producing it. We already detect IL-23p19 message from the lungs of exposed mice following three weeks of SR exposure. As discussed earlier, $\mathrm{TNF} \alpha$ plays an important role in the maintenance granuloma integrity. The significant decrease in TNF $\alpha$ from TLR2/9 DKO BMDMs might explain the dampened the inflammatory environment in the lungs of TLR2/9 DKO mice. Also, it has been reported that TNF $\alpha$, IL-16, and IL-17A stimulates chemokines required for migration of Th17 cells from endometriotic stromal cells [217]. Consequently, TNF $\alpha$ could play an indirect role in recruitment of Th17 cells into the lungs through induction of chemokines required for Th17 cells attraction. In conclusion, these results indicated TLRs 2 and 9 play an important role in the induction of these cytokines from BMDMs. 


\section{CHAPTER 5. DISCUSSION}

\section{HP IS ASSOCIATED WITH A PREDOMINANT TH17 RESPONSE}

The results from our studies demonstrated that granulomatous HP was associated with a Th17 response. Following three weeks of SR exposure (granulomatous HP), we found a predominant Th17 response associated with granuloma formation. Since we found Th17 response was predominant during granulomatous HP, we decided to examine $\mathrm{T}$ cell response during chronic HP. Following twenty five weeks of SR exposure, we found a strong Th17 response. In conclusion our results revealed that both granulomatous and chronic HP were associated with Th7 response.

Although the results demonstrated that HP was associated with Th17 response, the studies were only showing a correlation between HP and Th17 response not a cause and effect relationship. To address the role of Th17 cells in HP, we could adoptively transfer Th17 cells into RAG-2 KO mice then expose the mice to SR for three weeks. Finally, we will measure parameters of disease severity such as granuloma formation and fibrosis. Simonian et al., also reported that HP is associated with Th17 response and they are required for lung inflammation and fibrosis.

\section{CORRELATION BETWEEN T-BET, DISEASE SEVERITY AND TH17 RESPONSE DURING HP}

Despite the fact that Th17 was the dominant response during HP, there were a small percentage of SR specific Th1 cells in the lungs. However, we were not sure what is the role of these cells during HP? In our lab, previous studies showed that IFN $\gamma$ produced from Th1 cells was not necessary for granuloma formation calling into the role of Th1 cells. To address the role of Th1 in HP we used T-bet KO mice. Following 3 weeks of SR exposure, we found upregulation in IL-17 mRNA and protein expression in T-bet KO exposed mice compared to WT exposed mice. Also we found an increase in the frequency of Th17 cells accompanied by exacerbated peribronchiolar granulomatous inflammation in T-bet KO exposed mice compared to WT exposed mice. Our findings were confirmed by other studies in the literature. For example, T-bet KO mice infected with SEA/CFA showed increase in disease severity accompanied by increase in Th17 cytokines [169]. Furthermore, in T. cruzi mouse infection model there was an increase in the percentage of Th17 cells from the spleens of T-bet KO mice [170]. In conclusion, our studies revealed the protective role of T-bet during HP and its inhibitory effect on Th17 response.

Despite the protective role of T-bet and its inhibitory effect on Th17 response during HP, we did not address the type of the immune cell expressing T-bet that is responsible for these effects. To fulfill this aim, we could adoptively transfer either WT or T-bet $\mathrm{KO}$ naïve $\mathrm{CD} 4{ }^{+} \mathrm{T}$ cells into RAG-2 $\mathrm{KO}$ mice then expose the mice for three weeks and measure parameters for disease severity and Th17 response. If we do see the 
same degree of disease severity in both groups, therefore, T-bet in T cells is not responsible for disease severity. If we find disease severity in the group receiving T-bet KO T cells is more than mice receiving WT T cells, therefore, T-bet regulate disease severity in a T cell intrinisic manner. In T. cruzi mouse infection model, T-bet regulates Th17 development in $\mathrm{T}$ cell intrinisic manner. The authors showed the adoptive transfer of naïve T-bet $\mathrm{KO} \mathrm{CD}^{+} \mathrm{T}$ cells into RAG-2 $\mathrm{KO}$ enhance expression of IL-17 from $\mathrm{CD} 4^{+}$ T cells more than WT transfer to RAG-2 KO mice [170].

\section{EFFECT OF TLRS 2 AND 9 ON TH17 RESPONSE DURING HP}

The results from the previous two sections revealed that HP was associated with a predominant Th17 response and T-bet controls Th17 response and disease severity during HP. Consequently, we were looking for other factors that could regulate Th17 response during HP and we came across the following observation from previous studies in our lab. Following three weeks of SR exposure, the frequency of Th17 cells was fewer in the lungs of TLR2/9 DKO mice compared to WT mice. We were investigating the reason behind this observation and we found several possibilities that can be hypothesized. We tested one possibility which was inability of antigen presenting cells (APCs) to present $\mathrm{SR}$ antigen to effector $\mathrm{T}$ cell in the lungs of TLR2/9DKO. Since antigen presentation starts with the uptake of the bacteria, we tested first the phagocytosis ability of WT and TLR2/9 DKO APCs in vitro and in vivo. Our results revealed that both WT and TLR2/9 DKO BMDMs or BMDCs were capable of SR uptake in vitro. To confirm the in vitro studies, we performed in vivo phagocytosis to test the phagocytosis ability of alveolar macrophages using labeled SR. The data have demonstrated that both WT and TLR2/9 DKO alveolar macrophages are equally efficient in phagocytosis. In conclusion these results showed that absence of TLRs 2 and 9 does not affect the phagocytosis ability of APCs. Next we examined the antigen presentation ability of WT and TLR2/9 DKO APCs to check if they were capable of presenting SR antigen to effector Th17 cells. We found

that both WT and TLR2/9 DKO APCs were equally efficient in presenting SR antigen to WT and TLR2/9 DKO effector T cells. In summary, TLRs 2 and 9 did not have an effect on antigen presentation ability of the APCs. 


\section{CHAPTER 6. CONCLUDING REMARKS}

HP is a T cell-mediated disease and several studies have shown the pathogenic role of T cells during HP. Consequently, our studies were focused mainly on determining the type of $\mathrm{T}$ cell response and unraveling some of the factors that might control $\mathrm{T}$ cell response during HP. Targeting these factors might be a potential future therapy for HP.

An overall summary for our studies is depicted in Fig. 6-1. Once SR is inhaled, it stimulates lung immune and non-immune cells to produce cytokines and chemokines in MyD88-dependant fashion. CXCL1 and CXCL2 are chemokines required for neutrophil recruitment. Following the first wave of cytokines and chemokines, neutrophils were recruited into the airways. Neutrophils were considered as a crucial source of IFN $\gamma$, which is required for granuloma formation in this model. Our studies demonstrated that neutrophils produce IFN $\gamma$ in an IL-12/IL-18, or IL-15/IL-18-dependant manner.

Dendritic cells can sense SR in the airways by their dendrites. Consequently, they undergo a maturation process and migrate to lymph nodes, starting polarization of naïve $\mathrm{T}$ cells into effector $\mathrm{T}$ cells. We found that the predominant $\mathrm{T}$ cell response in the lungs during HP was a Th17 response with minimal Th1 response. We also found that T-bet regulates Th17 response as well as granuloma formation during HP. Therefore, we consider T-bet one of the factors that regulates Th17 response as well as granuloma formation and lung fibrosis. Based on these results, we might consider T-bet as a therapeutic target for HP treatment. Polymorphisms in T-bet could explain why some patients develop fibrosis while others do not. Sequencing T-bet from a number of HP patients at the chronic stages and comparing them to patients at granulomatous stages as well as normal subjects could give us insights into how T-bet polymorphisms decide the fate of HP.

We also found from previous studies in our lab that TLRs control the Th17 response during granulomatous HP; however, the mechanism(s) behind this control is still under investigation. So far we found that TLR2 and 9 did not affect the phagocytosis ability of macrophages and dendritic cells. Furthermore, TLRs2 and 9 did not affect the antigen presentation ability of APCs. Even though we still lack the exact mechanism of controlling Th17 response by TLR2 and 9, that doesn't eliminate the idea of TLRs as therapeutic targets. Again TLRs polymorphisms could play a role in the severity of HP. 


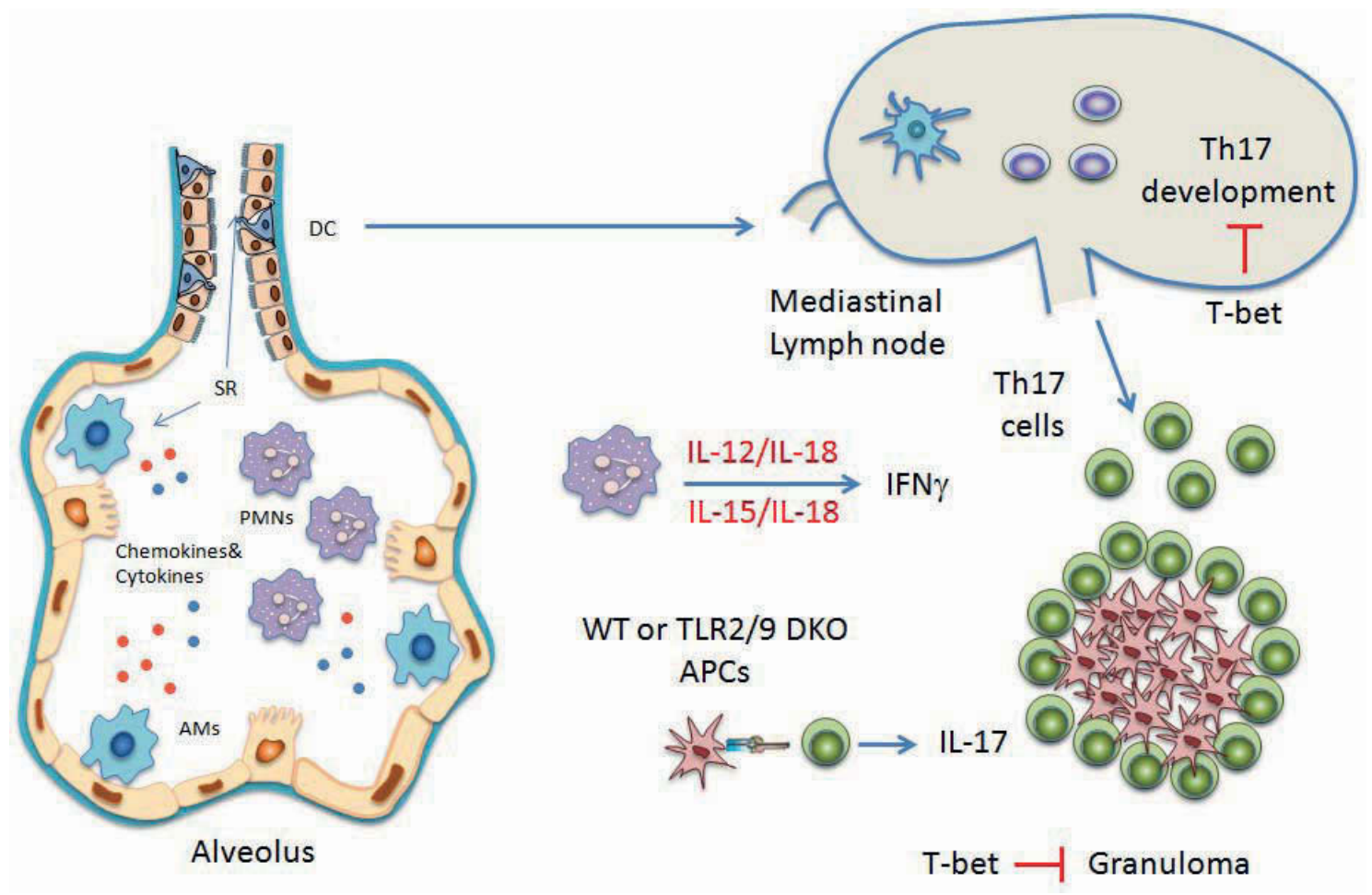

Figure 6-1. Overall HP model.

SR stimulates AMs and probably other cells in the airways to produce different chemokines and cytokines that recruit neutrophils into airways. SR also could be detected by DCs which consequently migrate to lymph nodes to initiate polarization of naïve $\mathrm{T}$ cells into effector Th17 cells. Th17 effector T cells migrate to the lungs for granuloma formation. T-bet inhibited both Th17 development and granuloma formation. Both WT and TLR2/9 DKO APCs were able to present SR antigen to Th17 cells, stimulating IL-17 production from Th17 cells. 


\section{LIST OF REFERENCES}

1. Kobayashi, K., K. Kaneda, and T. Kasama, Immunopathogenesis of delayed-type hypersensitivity. Microsc Res Tech, 2001. 53(4): p. 241-5.

2. Rajan, T.V., The Gell-Coombs classification of hypersensitivity reactions: a reinterpretation. Trends Immunol, 2003. 24(7): p. 376-9.

3. Mohr, L.C., Hypersensitivity pneumonitis. Curr Opin Pulm Med, 2004. 10(5): p. 401-11.

4. Girard, M., Y. Lacasse, and Y. Cormier, Hypersensitivity pneumonitis. Allergy, 2009. 64(3): p. 322-34.

5. Banchereau, J. and R.M. Steinman, Dendritic cells and the control of immunity. Nature, 1998. 392(6673): p. 245-52.

6. Schwartz, R.H., A cell culture model for T lymphocyte clonal anergy. Science, 1990. 248(4961): p. 1349-56.

7. Schwartz, R.H., Costimulation of T lymphocytes: the role of CD28, CTLA-4, and B7/BB1 in interleukin-2 production and immunotherapy. Cell, 1992. 71(7): $\mathrm{p}$. $1065-8$.

8. Szabo, S.J., et al., A novel transcription factor, T-bet, directs Th1 lineage commitment. Cell, 2000. 100(6): p. 655-69.

9. Lugo-Villarino, G., et al., T-bet is required for optimal production of IFN-gamma and antigen-specific T cell activation by dendritic cells. Proc Natl Acad Sci U S A, 2003. 100(13): p. 7749-54.

10. Townsend, M.J., et al., T-bet regulates the terminal maturation and homeostasis of NK and Valpha14i NKT cells. Immunity, 2004. 20(4): p. 477-94.

11. Mullen, A.C., et al., Role of T-bet in commitment of TH1 cells before IL-12dependent selection. Science, 2001. 292(5523): p. 1907-10.

12. Afkarian, M., et al., T-bet is a STAT1-induced regulator of IL-12R expression in naive CD4+ T cells. Nat Immunol, 2002. 3(6): p. 549-57.

13. Lighvani, A.A., et al., T-bet is rapidly induced by interferon-gamma in lymphoid and myeloid cells. Proc Natl Acad Sci U S A, 2001. 98(26): p. 15137-42.

14. Zhu, J., H. Yamane, and W.E. Paul, Differentiation of effector CD4 T cell populations (*). Annu Rev Immunol, 2010. 28: p. 445-89. 
15. Veldhoen, M., et al., TGFbeta in the context of an inflammatory cytokine milieu supports de novo differentiation of IL-17-producing T cells. Immunity, 2006. 24(2): p. 179-89.

16. Kimura, A., T. Naka, and T. Kishimoto, IL-6-dependent and-independent pathways in the development of interleukin 17-producing Thelper cells. Proc Natl Acad Sci U S A, 2007. 104(29): p. 12099-104.

17. Mangan, P.R., et al., Transforming growth factor-beta induces development of the $T(H) 17$ lineage. Nature, 2006. 441(7090): p. 231-4.

18. Bettelli, E., et al., Reciprocal developmental pathways for the generation of pathogenic effector TH17 and regulatory T cells. Nature, 2006. 441(7090): p. 235-8.

19. Ivanov, II, et al., The orphan nuclear receptor RORgammat directs the differentiation program of proinflammatory IL-17+ T helper cells. Cell, 2006. 126(6): p. 1121-33.

20. Harrington, L.E., et al., Interleukin 17-producing CD4+ effector T cells develop via a lineage distinct from the Thelper type 1 and 2 lineages. Nat Immunol, 2005. 6(11): p. 1123-32.

21. Park, H., et al., A distinct lineage of CD4 T cells regulates tissue inflammation by producing interleukin 17. Nat Immunol, 2005. 6(11): p. 1133-41.

22. Yang, X.O., et al., Thelper 17 lineage differentiation is programmed by orphan nuclear receptors ROR alpha and ROR gamma. Immunity, 2008. 28(1): p. 29-39.

23. Huang, W., et al., Requirement of interleukin-17A for systemic anti-Candida albicans host defense in mice. J Infect Dis, 2004. 190(3): p. 624-31.

24. Khader, S.A., et al., IL-23 and IL-17 in the establishment of protective pulmonary CD4+ T cell responses after vaccination and during Mycobacterium tuberculosis challenge. Nat Immunol, 2007. 8(4): p. 369-77.

25. Wu, Q., et al., IL-23-dependent IL-17 production is essential in neutrophil recruitment and activity in mouse lung defense against respiratory Mycoplasma pneumoniae infection. Microbes Infect, 2007. 9(1): p. 78-86.

26. Higgins, S.C., et al., TLR4 mediates vaccine-induced protective cellular immunity to Bordetella pertussis: role of IL-17-producing T cells. J Immunol, 2006. 177(11): p. 7980-9.

27. Happel, K.I., et al., Divergent roles of $I L-23$ and IL-12 in host defense against Klebsiella pneumoniae. J Exp Med, 2005. 202(6): p. 761-9. 
28. Aujla, S.J., et al., IL-22 mediates mucosal host defense against Gram-negative bacterial pneumonia. Nat Med, 2008. 14(3): p. 275-81.

29. Lin, Y., et al., Interleukin-17 is required for Thelper 1 cell immunity and host resistance to the intracellular pathogen Francisella tularensis. Immunity, 2009. 31(5): p. 799-810.

30. Zheng, Y., et al., Interleukin-22 mediates early host defense against attaching and effacing bacterial pathogens. Nat Med, 2008. 14(3): p. 282-9.

31. Bettelli, E., et al., Induction and effector functions of $T(H) 17$ cells. Nature, 2008. 453(7198): p. 1051-7.

32. Sato, K., et al., Th17 functions as an osteoclastogenic helper T cell subset that links T cell activation and bone destruction. J Exp Med, 2006. 203(12): p. 267382.

33. Kebir, H., et al., Human TH17 lymphocytes promote blood-brain barrier disruption and central nervous system inflammation. Nat Med, 2007. 13(10): p. 1173-5.

34. Chabaud, M., et al., Human interleukin-17: A T cell-derived proinflammatory cytokine produced by the rheumatoid synovium. Arthritis Rheum, 1999. 42(5): p. 963-70.

35. Joosten, L.A., et al., Association of interleukin-18 expression with enhanced levels of both interleukin-1beta and tumor necrosis factor alpha in knee synovial tissue of patients with rheumatoid arthritis. Arthritis Rheum, 2003. 48(2): p. 339-47.

36. Fujino, S., et al., Increased expression of interleukin 17 in inflammatory bowel disease. Gut, 2003. 52(1): p. 65-70.

37. Nakae, S., et al., Suppression of immune induction of collagen-induced arthritis in IL-17-deficient mice. J Immunol, 2003. 171(11): p. 6173-7.

38. Nakae, S., et al., IL-17 production from activated $T$ cells is required for the spontaneous development of destructive arthritis in mice deficient in $I L-1$ receptor antagonist. Proc Natl Acad Sci U S A, 2003. 100(10): p. 5986-90.

39. Horwitz, D.A., S.G. Zheng, and J.D. Gray, Natural and TGF-beta-induced Foxp3(+)CD4(+) CD25(+) regulatory $T$ cells are not mirror images of each other. Trends Immunol, 2008. 29(9): p. 429-35.

40. Littman, D.R. and A.Y. Rudensky, Th17 and regulatory T cells in mediating and restraining inflammation. Cell, 2010. 140(6): p. 845-58.

41. Vignali, D.A., L.W. Collison, and C.J. Workman, How regulatory T cells work. Nat Rev Immunol, 2008. 8(7): p. 523-32. 
42. Co, D.O., et al., T cell contributions to the different phases of granuloma formation. Immunol Lett, 2004. 92(1-2): p. 135-42.

43. Miller, E.A. and J.D. Ernst, Illuminating the black box of TNF action in tuberculous granulomas. Immunity, 2008. 29(2): p. 175-7.

44. Schreiber, H.A. and M. Sandor, The role of dendritic cells in mycobacteriuminduced granulomas. Immunol Lett, 2010. 130(1-2): p. 26-31.

45. Sandor, M., J.V. Weinstock, and T.A. Wynn, Granulomas in schistosome and mycobacterial infections: a model of local immune responses. Trends Immunol, 2003. 24(1): p. 44-52.

46. Barnes, P.F., et al., Tuberculosis in patients with human immunodeficiency virus infection. N Engl J Med, 1991. 324(23): p. 1644-50.

47. Scanga, C.A., et al., Depletion of CD4(+) T cells causes reactivation of murine persistent tuberculosis despite continued expression of interferon gamma and nitric oxide synthase 2. J Exp Med, 2000. 192(3): p. 347-58.

48. Ladel, C.H., et al., Studies with MHC-deficient knock-out mice reveal impact of both MHC I- and MHC II-dependent T cell responses on Listeria monocytogenes infection. J Immunol, 1994. 153(7): p. 3116-22.

49. Fallon, P.G., et al., Elevated type 1, diminished type 2 cytokines and impaired antibody response are associated with hepatotoxicity and mortalities during Schistosoma mansoni infection of CD4-depleted mice. Eur J Immunol, 2000. 30(2): p. 470-80.

50. Hogan, L.H., et al., CD4+ TCR repertoire heterogeneity in Schistosoma mansoniinduced granulomas. J Immunol, 2002. 169(11): p. 6386-93.

51. Kindler, V., et al., The inducing role of tumor necrosis factor in the development of bactericidal granulomas during BCG infection. Cell, 1989. 56(5): p. 731-40.

52. Zganiacz, A., et al., TNF-alpha is a critical negative regulator of type 1 immune activation during intracellular bacterial infection. J Clin Invest, 2004. 113(3): p. 401-13.

53. Roach, D.R., et al., TNF regulates chemokine induction essential for cell recruitment, granuloma formation, and clearance of mycobacterial infection. $\mathrm{J}$ Immunol, 2002. 168(9): p. 4620-7.

54. Cooper, A.M., et al., Disseminated tuberculosis in interferon gamma genedisrupted mice. J Exp Med, 1993. 178(6): p. 2243-7. 
55. Clay, H., H.E. Volkman, and L. Ramakrishnan, Tumor necrosis factor signaling mediates resistance to mycobacteria by inhibiting bacterial growth and macrophage death. Immunity, 2008. 29(2): p. 283-94.

56. Algood, H.M., et al., TNF influences chemokine expression of macrophages in vitro and that of CD11b+cells in vivo during Mycobacterium tuberculosis infection. J Immunol, 2004. 172(11): p. 6846-57.

57. Chakravarty, S.D., et al., Tumor necrosis factor blockade in chronic murine tuberculosis enhances granulomatous inflammation and disorganizes granulomas in the lungs. Infect Immun, 2008. 76(3): p. 916-26.

58. Egen, J.G., et al., Macrophage and T cell dynamics during the development and disintegration of mycobacterial granulomas. Immunity, 2008. 28(2): p. 271-84.

59. Mohan, V.P., et al., Effects of tumor necrosis factor alpha on host immune response in chronic persistent tuberculosis: possible role for limiting pathology. Infect Immun, 2001. 69(3): p. 1847-55.

60. Keane, J., B. Shurtleff, and H. Kornfeld, TNF-dependent BALB/c murine macrophage apoptosis following Mycobacterium tuberculosis infection inhibits bacillary growth in an IFN-gamma independent manner. Tuberculosis (Edinb), 2002. 82(2-3): p. 55-61.

61. Wynn, T.A., Integrating mechanisms of pulmonary fibrosis. J Exp Med, 2011. 208(7): p. 1339-50.

62. Simonian, P.L., et al., Th17-polarized immune response in a murine model of hypersensitivity pneumonitis and lung fibrosis. J Immunol, 2009. 182(1): p. 65765.

63. Wilson, M.S., et al., Bleomycin and IL-1beta-mediated pulmonary fibrosis is IL17A dependent. J Exp Med, 2010. 207(3): p. 535-52.

64. $\mathrm{Xu}, \mathrm{J}$. , et al., Increased bleomycin-induced lung injury in mice deficient in the transcription factor T-bet. Am J Physiol Lung Cell Mol Physiol, 2006. 291(4): p. L658-67.

65. Aliprantis, A.O., et al., Transcription factor T-bet regulates skin sclerosis through its function in innate immunity and via IL-13. Proc Natl Acad Sci U S A, 2007. 104(8): p. 2827-30.

66. Hirschmann, J.V., S.N. Pipavath, and J.D. Godwin, Hypersensitivity pneumonitis: a historical, clinical, and radiologic review. Radiographics, 2009. 29(7): p. 192138.

67. Towey, J.W., Henery C. Sweany, Willis H. Huron, Severe Bronchial Asthma apparently due to Fungus Spores found in Maple Bark. JAMA, 1932. 99(6): p. 7. 
68. Campbell, J., Acute symptoms following work with hay. BMJ, 1932. 2.

69. Pepys, J., Hypersensitivity diseases of the lungs due to fungi and organic dusts. Monographs in allergy,. 1969, Basel, New York,: Karger. viii, 147 p.

70. Selman, M., Hypersensitivity pneumonitis: a multifaceted deceiving disorder. Clin Chest Med, 2004. 25(3): p. 531-47, vi.

71. Fink, J.N., Hypersensitivity pneumonitis. J Allergy Clin Immunol, 1984. 74(1): p. $1-10$.

72. Fink, J.N., et al., Needs and opportunities for research in hypersensitivity pneumonitis. Am J Respir Crit Care Med, 2005. 171(7): p. 792-8.

73. Pepys, J., et al., Farmer's Lung. Thermophilic Actinomycetes as a Source of "Farmer's Lung Hay" Antigen. Lancet, 1963. 2(7308): p. 607-11.

74. Kurup, V.P. and N.S. Agre, Transfer of Micropolyspora rectivirgula (Karssil'nikov and Agre) Lechevalier and Becker 1966 to Faenia gen. nov. International Journal of Systemic Bacteriology, 1983. 33.

75. Reboux, G., et al., Role of molds in farmer's lung disease in Eastern France. Am J Respir Crit Care Med, 2001. 163(7): p. 1534-9.

76. Gbaguidi-Haore, H., et al., Multilevel analysis of the impact of environmental factors and agricultural practices on the concentration in hay of microorganisms responsible for farmer's lung disease. Ann Agric Environ Med, 2009. 16(2): p. 219-25.

77. Dhar, S. and F. Daroowalla, Hypersenitivity Pneumonitis. Clinical Pulmonary Medicine, 2011. 18(4): p. 169-174.

78. Morell, F., et al., Bird fancier's lung: a series of 86 patients. Medicine (Baltimore), 2008. 87(2): p. 110-30.

79. Moreno-Ancillol, A., et al., Hypersensitivity pneumonitis due to occupational inhalation of fungi-contaminated corn dust. J Investig Allergol Clin Immunol, 2004. 14(2): p. 165-7.

80. Channell, S., et al., Allergic alveolitis in maltworkers. A clinical, mycological, and immunological study. Q J Med, 1969. 38(152): p. 351-76.

81. Campbell, J.A., et al., Cheese worker's hypersensitivity pneumonitis. Am Rev Respir Dis, 1983. 127(4): p. 495-6.

82. Cormier, Y., et al., Hypersensitivity pneumonitis in peat moss processing plant workers. Am J Respir Crit Care Med, 1998. 158(2): p. 412-7. 
83. Morell, F., et al., Suberosis: clinical study and new etiologic agents in a series of eight patients. Chest, 2003. 124(3): p. 1145-52.

84. van Heemst, R.C., et al., Hypersensitivity pneumonitis caused by occupational exposure to phytase. Eur Respir J, 2009. 33(6): p. 1507-9.

85. Guillot, M., et al., [Dry sausage mould hypersensitivity pneumonitis: three cases]. Rev Mal Respir, 2008. 25(5): p. 596-600.

86. Lopez, M. and J.E. Salvaggio, Epidemiology of hypersensitivity pneumonitis/allergic alveolitis. Monogr Allergy, 1987. 21: p. 70-86.

87. Richerson, H.B., et al., Guidelines for the clinical evaluation of hypersensitivity pneumonitis. Report of the Subcommittee on Hypersensitivity Pneumonitis. J Allergy Clin Immunol, 1989. 84(5 Pt 2): p. 839-44.

88. Schuyler, M. and Y. Cormier, The diagnosis of hypersensitivity pneumonitis. Chest, 1997. 111(3): p. 534-6.

89. Takemura, T., et al., Pathology of hypersensitivity pneumonitis. Curr Opin Pulm Med, 2008. 14(5): p. 440-54.

90. Sharma, O.P. and N. Fujimura, Hypersensitivity pneumonitis: a noninfectious granulomatosis. Semin Respir Infect, 1995. 10(2): p. 96-106.

91. Lacasse, Y., et al., Classification of hypersensitivity pneumonitis: a hypothesis. Int Arch Allergy Immunol, 2009. 149(2): p. 161-6.

92. Lacasse, Y., et al., Clinical diagnosis of hypersensitivity pneumonitis. Am J Respir Crit Care Med, 2003. 168(8): p. 952-8.

93. Hodgson, M.J., D.K. Parkinson, and M. Karpf, Chest X-rays in hypersensitivity pneumonitis: a metaanalysis of secular trend. Am J Ind Med, 1989. 16(1): p. 4553.

94. Roberts, R.C., F.J. Wenzel, and D.A. Emanuel, Precipitating antibodies in a midwest dairy farming population toward the antigens associated with farmer's lung disease. J Allergy Clin Immunol, 1976. 57(6): p. 518-24.

95. McSharry, C., et al., Antibody measurement in extrinsic allergic alveolitis. Eur J Respir Dis, 1984. 65(4): p. 259-65.

96. Krasnick, J., et al., Hypersensitivity pneumonitis: problems in diagnosis. J Allergy Clin Immunol, 1996. 97(4): p. 1027-30.

97. McSharry, C., S.W. Banham, and G. Boyd, Effect of cigarette smoking on the antibody response to inhaled antigens and the prevalence of extrinsic allergic alveolitis among pigeon breeders. Clin Allergy, 1985. 15(5): p. 487-94. 
98. Anderson, K., et al., Effect of cigarette smoking on the specific antibody response in pigeon fanciers. Thorax, 1988. 43(10): p. 798-800.

99. Baldwin, C.I., et al., Pigeon fanciers' lung: effects of smoking on serum and salivary antibody responses to pigeon antigens. Clin Exp Immunol, 1998. 113(2): p. 166-72.

100. Hirata, K., H. Kanazawa, and H. Kamoi, IV. Clinical aspects of delayed hypersensitivity in lungs: pathophysiology of hypersensitivity disorders in clinics. Microsc Res Tech, 2001. 53(4): p. 307-12.

101. Meyer, K.C., Bronchoalveolar lavage as a diagnostic tool. Semin Respir Crit Care Med, 2007. 28(5): p. 546-60.

102. Fournier, E., et al., Early neutrophil alveolitis after antigen inhalation in hypersensitivity pneumonitis. Chest, 1985. 88(4): p. 563-6.

103. Yi, E.S., Hypersensitivity pneumonitis. Crit Rev Clin Lab Sci, 2002. 39(6): p. 581-629.

104. Coultas, D.B., et al., The epidemiology of interstitial lung diseases. Am J Respir Crit Care Med, 1994. 150(4): p. 967-72.

105. Gruchow, H.W., et al., Precipitating antibodies to farmer's lung antigens in a Wisconsin farming population. Am Rev Respir Dis, 1981. 124(4): p. 411-5.

106. Fenclova, Z., et al., Occupational hypersensitivity pneumonitis reported to the Czech National Registry Of Occupational Diseases in the period 1992-2005. Ind Health, 2009. 47(4): p. 443-8.

107. McDonald, J.C., et al., Incidence by occupation and industry of acute work related respiratory diseases in the UK, 1992-2001. Occup Environ Med, 2005. 62(12): p. 836-42.

108. Camarena, A., et al., Major histocompatibility complex and tumor necrosis factoralpha polymorphisms in pigeon breeder's disease. Am J Respir Crit Care Med, 2001. 163(7): p. 1528-33.

109. Camarena, A., et al., PSMB8 (LMP7) but not PSMB9 (LMP2) gene polymorphisms are associated to pigeon breeder's hypersensitivity pneumonitis. Respir Med, 2010. 104(6): p. 889-94.

110. Sugiyama, Y., et al., Chemokines in bronchoalveolar lavage fluid in summer-type hypersensitivity pneumonitis. Eur Respir J, 1995. 8(7): p. 1084-90.

111. Denis, M., Proinflammatory cytokines in hypersensitivity pneumonitis. Am J Respir Crit Care Med, 1995. 151(1): p. 164-9. 
112. Fujishima, S., et al., Flow cytometric detection of cell-associated interleukin-8 in alveolar macrophages in vivo from patients with hypersensitivity pneumonitis and sarcoidosis. Scand J Clin Lab Invest, 2004. 64(3): p. 237-43.

113. Pardo, A., et al., Increase of lung neutrophils in hypersensitivity pneumonitis is associated with lung fibrosis. Am J Respir Crit Care Med, 2000. 161(5): p. 1698704.

114. Ye, Q., et al., Interleukin 12, interleukin 18, and tumor necrosis factor alpha release by alveolar macrophages: acute and chronic hypersensitivity pneumonitis. Ann Allergy Asthma Immunol, 2009. 102(2): p. 149-54.

115. Suga, M., et al., Mechanisms accounting for granulomatous responses in hypersensitivity pneumonitis. Sarcoidosis Vasc Diffuse Lung Dis, 1997. 14(2): p. 131-8.

116. Agostini, C., et al., CXCR3/CXCL10 interactions in the development of hypersensitivity pneumonitis. Respir Res, 2005. 6: p. 20.

117. Costabel, U., et al., T-lymphocytosis in bronchoalveolar lavage fluid of hypersensitivity pneumonitis. Changes in profile of T-cell subsets during the course of disease. Chest, 1984. 85(4): p. 514-22.

118. Murayama, J., et al., Lung fibrosis in hypersensitivity pneumonitis. Association with $C D 4+$ but not $C D 8+$ cell dominant alveolitis and insidious onset. Chest, 1993. 104(1): p. 38-43.

119. Barrera, L., et al., Functional diversity of T-cell subpopulations in subacute and chronic hypersensitivity pneumonitis. Am J Respir Crit Care Med, 2008. 177(1): p. 44-55.

120. Ando, M., et al., Difference in the phenotypes of bronchoalveolar lavage lymphocytes in patients with summer-type hypersensitivity pneumonitis, farmer's lung, ventilation pneumonitis, and bird fancier's lung: report of a nationwide epidemiologic study in Japan. J Allergy Clin Immunol, 1991. 87(5): p. 1002-9.

121. Agostini, C., et al., Pulmonary alveolar macrophages in patients with sarcoidosis and hypersensitivity pneumonitis: characterization by monoclonal antibodies. $\mathrm{J}$ Clin Immunol, 1987. 7(1): p. 64-70.

122. Israel-Assayag, E., et al., Expression of costimulatory molecules on alveolar macrophages in hypersensitivity pneumonitis. Am J Respir Crit Care Med, 1999. 159(6): p. 1830-4.

123. Pforte, A., et al., Expression of the adhesion molecule ICAM-1 on alveolar macrophages and in serum in extrinsic allergic alveolitis. Respiration, 1993. 60(4): p. 221-6. 
124. Schuyler, M., K. Gott, and A. Cherne, Mediators of hypersensitivity pneumonitis. J Lab Clin Med, 2000. 136(1): p. 29-38.

125. Denis, M. and E. Ghadirian, Transforming growth factor-beta is generated in the course of hypersensitivity pneumonitis: contribution to collagen synthesis. Am J Respir Cell Mol Biol, 1992. 7(2): p. 156-60.

126. Denis, M., et al., Tumor necrosis factor plays an essential role in determining hypersensitivity pneumonitis in a mouse model. Am J Respir Cell Mol Biol, 1991. 5(5): p. 477-83.

127. Nance, S.C., et al., MyD88 is necessary for neutrophil recruitment in hypersensitivity pneumonitis. J Leukoc Biol, 2008. 83(5): p. 1207-17.

128. Gudmundsson, G. and G.W. Hunninghake, Interferon-gamma is necessary for the expression of hypersensitivity pneumonitis. J Clin Invest, 1997. 99(10): p. 238690.

129. Nance, S., et al., IFN-gamma production by innate immune cells is sufficient for development of hypersensitivity pneumonitis. Eur J Immunol, 2005. 35(6): p. 1928-38.

130. Nance, S., R. Cross, and E. Fitzpatrick, Chemokine production during hypersensitivity pneumonitis. Eur J Immunol, 2004. 34(3): p. 677-85.

131. Gudmundsson, G., et al., Interleukin-10 modulates the severity of hypersensitivity pneumonitis in mice. Am J Respir Cell Mol Biol, 1998. 19(5): p. 812-8.

132. Klebanoff, S.J., et al., Stimulation of neutrophils by tumor necrosis factor. J Immunol, 1986. 136(11): p. 4220-5.

133. Gamble, J.R., et al., Stimulation of the adherence of neutrophils to umbilical vein endothelium by human recombinant tumor necrosis factor. Proc Natl Acad Sci U S A, 1985. 82(24): p. 8667-71.

134. Bachwich, P.R., et al., Tumor necrosis factor stimulates interleukin-1 and prostaglandin E2 production in resting macrophages. Biochem Biophys Res Commun, 1986. 136(1): p. 94-101.

135. Schkade, P.A. and J.M. Routes, Hypersensitivity pneumonitis in a patient with hypogammaglobulinemia. J Allergy Clin Immunol, 1996. 98(3): p. 710-2.

136. Ohta, K., et al., Hypersensitivity pneumonitis in the athymic nude mouse. Another evidence of T cell dependency. Chest, 1993. 103(2 Suppl): p. 145S.

137. Schuyler, M., et al., CD3+, CD4+, CD8-, Ia- T cells adoptively transfer murine experimental hypersensitivity pneumonitis. Chest, 1993. 103(2 Suppl): p. 143S$145 \mathrm{~S}$. 
138. Schuyler, M., et al., Experimental hypersensitivity pneumonitis. Effect of CD4 cell depletion. Am J Respir Crit Care Med, 1994. 149(5): p. 1286-94.

139. Gudmundsson, G., M.M. Monick, and G.W. Hunninghake, IL-12 modulates expression of hypersensitivity pneumonitis. J Immunol, 1998. 161(2): p. 991-9.

140. Butler, N.S., et al., Altered IL-4 mRNA stability correlates with Th1 and Th2 bias and susceptibility to hypersensitivity pneumonitis in two inbred strains of mice. $\mathrm{J}$ Immunol, 2002. 169(7): p. 3700-9.

141. Matsuno, Y., et al., Overexpression of GATA-3 protects against the development of hypersensitivity pneumonitis. Am J Respir Crit Care Med, 2007. 176(10): p. 1015-25.

142. Schuyler, M., et al., Th1 CD4+ cells adoptively transfer experimental hypersensitivity pneumonitis. Cell Immunol, 1997. 177(2): p. 169-75.

143. Veldhoen, M., et al., Signals mediated by transforming growth factor-beta initiate autoimmune encephalomyelitis, but chronic inflammation is needed to sustain disease. Nat Immunol, 2006. 7(11): p. 1151-6.

144. Korn, T., et al., IL-21 initiates an alternative pathway to induce proinflammatory $T(H) 17$ cells. Nature, 2007. 448(7152): p. 484-7.

145. Nurieva, R., et al., Essential autocrine regulation by $I L-21$ in the generation of inflammatory T cells. Nature, 2007. 448(7152): p. 480-3.

146. Zhou, L., et al., IL-6 programs $T(H)-17$ cell differentiation by promoting sequential engagement of the IL-21 and IL-23 pathways. Nat Immunol, 2007. 8(9): p. 967-74.

147. Oppmann, B., et al., Novel p19 protein engages IL-12p40 to form a cytokine, IL23, with biological activities similar as well as distinct from IL-12. Immunity, 2000. 13(5): p. 715-25.

148. Stritesky, G.L., N. Yeh, and M.H. Kaplan, IL-23 promotes maintenance but not commitment to the Th17 lineage. J Immunol, 2008. 181(9): p. 5948-55.

149. Cua, D.J., et al., Interleukin-23 rather than interleukin-12 is the critical cytokine for autoimmune inflammation of the brain. Nature, 2003. 421(6924): p. 744-8.

150. Langrish, C.L., et al., IL-23 drives a pathogenic T cell population that induces autoimmune inflammation. J Exp Med, 2005. 201(2): p. 233-40.

151. Murphy, C.A., et al., Divergent pro- and antiinflammatory roles for $I L-23$ and IL12 in joint autoimmune inflammation. J Exp Med, 2003. 198(12): p. 1951-7. 
152. McGeachy, M.J., et al., TGF-beta and IL-6 drive the production of IL-17 and IL10 by T cells and restrain T(H)-17 cell-mediated pathology. Nat Immunol, 2007. 8(12): p. 1390-7.

153. Ghoreschi, K., et al., Generation of pathogenic $T(H) 17$ cells in the absence of TGF-beta signalling. Nature, 2010. 467(7318): p. 967-71.

154. El-Behi, M., et al., The encephalitogenicity of T(H)17 cells is dependent on IL-1and IL-23-induced production of the cytokine GM-CSF. Nat Immunol, 2011. 12(6): p. 568-75.

155. Codarri, L., et al., RORgammat drives production of the cytokine GM-CSF in helper T cells, which is essential for the effector phase of autoimmune neuroinflammation. Nat Immunol, 2011. 12(6): p. 560-7.

156. McQualter, J.L., et al., Granulocyte macrophage colony-stimulating factor: a new putative therapeutic target in multiple sclerosis. J Exp Med, 2001. 194(7): p. 87382.

157. Lockhart, E., A.M. Green, and J.L. Flynn, IL-17 production is dominated by gammadelta $T$ cells rather than CD4 T cells during Mycobacterium tuberculosis infection. J Immunol, 2006. 177(7): p. 4662-9.

158. Zhou, Q., et al., Cytokine profiling of macrophages exposed to Porphyromonas gingivalis, its lipopolysaccharide, or its FimA protein. Infect Immun, 2005. 73(2): p. 935-43.

159. Molet, S., et al., IL-17 is increased in asthmatic airways and induces human bronchial fibroblasts to produce cytokines. J Allergy Clin Immunol, 2001. 108(3): p. $430-8$.

160. Iwakura, Y., et al., Functional specialization of interleukin-17 family members. Immunity, 2011. 34(2): p. 149-62.

161. Laan, M., et al., Neutrophil recruitment by human $I L-17$ via $C$-X-C chemokine release in the airways. J Immunol, 1999. 162(4): p. 2347-52.

162. Miyamoto, M., et al., Endogenous IL-17 as a mediator of neutrophil recruitment caused by endotoxin exposure in mouse airways. J Immunol, 2003. 170(9): p. 4665-72.

163. Liang, S.C., et al., An IL-17F/A heterodimer protein is produced by mouse Th17 cells and induces airway neutrophil recruitment. J Immunol, 2007. 179(11): p. 7791-9.

164. Sonnenberg, G.F., L.A. Fouser, and D. Artis, Border patrol: regulation of immunity, inflammation and tissue homeostasis at barrier surfaces by IL-22. Nat Immunol, 2011. 12(5): p. 383-90. 
165. Boniface, K., et al., IL-22 inhibits epidermal differentiation and induces proinflammatory gene expression and migration of human keratinocytes. $\mathrm{J}$ Immunol, 2005. 174(6): p. 3695-702.

166. Brand, S., et al., IL-22 is increased in active Crohn's disease and promotes proinflammatory gene expression and intestinal epithelial cell migration. Am J Physiol Gastrointest Liver Physiol, 2006. 290(4): p. G827-38.

167. Zheng, Y., et al., Interleukin-22, a T(H)17 cytokine, mediates IL-23-induced dermal inflammation and acanthosis. Nature, 2007. 445(7128): p. 648-51.

168. Ma, H.L., et al., IL-22 is required for Th17 cell-mediated pathology in a mouse model of psoriasis-like skin inflammation. J Clin Invest, 2008. 118(2): p. 597-607.

169. Rutitzky, L.I., P.M. Smith, and M.J. Stadecker, T-bet protects against exacerbation of schistosome egg-induced immunopathology by regulating Th17mediated inflammation. Eur J Immunol, 2009. 39(9): p. 2470-81.

170. Guo, S., D. Cobb, and R.B. Smeltz, T-bet inhibits the in vivo differentiation of parasite-specific CD4+ Th17 cells in a T cell-intrinsic manner. J Immunol, 2009. 182(10): p. 6179-86.

171. Lexberg, M.H., et al., IFN-gamma and IL-12 synergize to convert in vivo generated Th17 into Th1/Th17 cells. Eur J Immunol, 2010. 40(11): p. 3017-27.

172. Hirota, K., et al., Fate mapping of IL-17-producing T cells in inflammatory responses. Nat Immunol, 2011. 12(3): p. 255-63.

173. Peters, A., Y. Lee, and V.K. Kuchroo, The many faces of Th17 cells. Curr Opin Immunol, 2011. 23(6): p. 702-6.

174. Joshi, A.D., et al., Interleukin-17-mediated immunopathogenesis in experimental hypersensitivity pneumonitis. Am J Respir Crit Care Med, 2009. 179(8): p. 70516.

175. Simonian, P.L., et al., gammadelta T cells protect against lung fibrosis via IL-22. J Exp Med, 2010. 207(10): p. 2239-53.

176. Martin, B., et al., Interleukin-17-producing gammadelta T cells selectively expand in response to pathogen products and environmental signals. Immunity, 2009. 31(2): p. 321-30.

177. Luger, D., et al., Either a Th17 or a Th1 effector response can drive autoimmunity: conditions of disease induction affect dominant effector category. $\mathrm{J}$ Exp Med, 2008. 205(4): p. 799-810. 
178. Murphy, A.C., et al., Infiltration of Th1 and Th17 cells and activation of microglia in the CNS during the course of experimental autoimmune encephalomyelitis. Brain Behav Immun, 2010. 24(4): p. 641-51.

179. Kroenke, M.A., et al., IL-12- and IL-23-modulated T cells induce distinct types of EAE based on histology, CNS chemokine profile, and response to cytokine inhibition. J Exp Med, 2008. 205(7): p. 1535-41.

180. O'Connor, R.A., et al., Cutting edge: Th1 cells facilitate the entry of Th17 cells to the central nervous system during experimental autoimmune encephalomyelitis. $\mathrm{J}$ Immunol, 2008. 181(6): p. 3750-4.

181. Lazarevic, V. and L.H. Glimcher, T-bet in disease. Nat Immunol, 2011. 12(7): p. 597-606.

182. Yeaman, G.R., et al., IFN-gamma is produced by polymorphonuclear neutrophils in human uterine endometrium and by cultured peripheral blood polymorphonuclear neutrophils. J Immunol, 1998. 160(10): p. 5145-53.

183. Ethuin, F., et al., Human neutrophils produce interferon gamma upon stimulation by interleukin-12. Lab Invest, 2004. 84(10): p. 1363-71.

184. Schroder, K., et al., Interferon-gamma: an overview of signals, mechanisms and functions. J Leukoc Biol, 2004. 75(2): p. 163-89.

185. Yu, C., et al., Expression of Th1/Th2 cytokine $m R N A$ in peritoneal exudative polymorphonuclear neutrophils and their effects on mononuclear cell Th1/Th2 cytokine production in MRL-lpr/lpr mice. Immunology, 1998. 95(3): p. 480-7.

186. Abdelsamed, H.A., et al., T-bet controls severity of hypersensitivity pneumonitis. J Inflamm (Lond), 2011. 8(1): p. 15.

187. Lazarevic, V., et al., T-bet represses T(H)17 differentiation by preventing Runx 1mediated activation of the gene encoding RORgammat. Nat Immunol, 2011. 12(1): p. 96-104.

188. Sullivan, B.M., et al., Increased susceptibility of mice lacking T-bet to infection with Mycobacterium tuberculosis correlates with increased IL-10 and decreased IFN-gamma production. J Immunol, 2005. 175(7): p. 4593-602.

189. Hultgren, O.H., M. Verdrengh, and A. Tarkowski, T-box transcription-factordeficient mice display increased joint pathology and failure of infection control during staphylococcal arthritis. Microbes Infect, 2004. 6(6): p. 529-35.

190. Ravindran, R., et al., Expression of T-bet by CD4 T cells is essential for resistance to Salmonella infection. J Immunol, 2005. 175(7): p. 4603-10. 
191. Bettelli, E., et al., Loss of T-bet, but not STAT1, prevents the development of experimental autoimmune encephalomyelitis. J Exp Med, 2004. 200(1): p. 79-87.

192. Wang, J., et al., Transcription factor T-bet regulates inflammatory arthritis through its function in dendritic cells. J Clin Invest, 2006. 116(2): p. 414-21.

193. Esensten, J.H., et al., T-bet-deficient NOD mice are protected from diabetes due to defects in both T cell and innate immune system function. J Immunol, 2009. 183(1): p. 75-82.

194. Juedes, A.E., et al., T-bet controls autoaggressive CD8 lymphocyte responses in type 1 diabetes. J Exp Med, 2004. 199(8): p. 1153-62.

195. Szabo, S.J., et al., Distinct effects of T-bet in TH1 lineage commitment and IFNgamma production in CD4 and CD8 T cells. Science, 2002. 295(5553): p. 338-42.

196. Lovett-Racke, A.E., et al., Silencing T-bet defines a critical role in the differentiation of autoreactive T lymphocytes. Immunity, 2004. 21(5): p. 719-31.

197. Koutoulaki, A., et al., TNFalpha and TGF-betal influence IL-18-induced IFNgamma production through regulation of $I L-18$ receptor and T-bet expression. Cytokine, 2010. 49(2): p. 177-84.

198. Lord, G.M., et al., T-bet is required for optimal proinflammatory CD4+ T-cell trafficking. Blood, 2005. 106(10): p. 3432-9.

199. Jenner, R.G., et al., The transcription factors T-bet and GATA-3 control alternative pathways of T-cell differentiation through a shared set of target genes. Proc Natl Acad Sci U S A, 2009. 106(42): p. 17876-81.

200. Munder, M., et al., Murine macrophages secrete interferon gamma upon combined stimulation with interleukin (IL)-12 and IL-18: A novel pathway of autocrine macrophage activation. J Exp Med, 1998. 187(12): p. 2103-8.

201. Trombetta, E.S. and I. Mellman, Cell biology of antigen processing in vitro and in vivo. Annu Rev Immunol, 2005. 23: p. 975-1028.

202. Stuart, L.M. and R.A. Ezekowitz, Phagocytosis and comparative innate immunity: learning on the fly. Nat Rev Immunol, 2008. 8(2): p. 131-41.

203. May, R.C. and L.M. Machesky, Phagocytosis and the actin cytoskeleton. J Cell Sci, 2001. 114(Pt 6): p. 1061-77.

204. Tan, S.Y. and M.K. Dee, Elie Metchnikoff (1845-1916): discoverer of phagocytosis. Singapore Med J, 2009. 50(5): p. 456-7.

205. Savina, A. and S. Amigorena, Phagocytosis and antigen presentation in dendritic cells. Immunol Rev, 2007. 219: p. 143-56. 
206. Kawai, T. and S. Akira, The role of pattern-recognition receptors in innate immunity: update on Toll-like receptors. Nat Immunol, 2010. 11(5): p. 373-84.

207. Blander, J.M. and R. Medzhitov, Regulation of phagosome maturation by signals from toll-like receptors. Science, 2004. 304(5673): p. 1014-8.

208. Blander, J.M. and R. Medzhitov, Toll-dependent selection of microbial antigens for presentation by dendritic cells. Nature, 2006. 440(7085): p. 808-12.

209. Albiger, B., et al., Toll-like receptor 9 acts at an early stage in host defence against pneumococcal infection. Cell Microbiol, 2007. 9(3): p. 633-44.

210. Shen, Y., et al., Toll-like receptor 2- and MyD88-dependent phosphatidylinositol 3-kinase and Racl activation facilitates the phagocytosis of Listeria monocytogenes by murine macrophages. Infect Immun, 2010. 78(6): p. 2857-67.

211. Shim, H.K., et al., Legionella lipoprotein activates toll-like receptor 2 and induces cytokine production and expression of costimulatory molecules in peritoneal macrophages. Exp Mol Med, 2009. 41(10): p. 687-94.

212. Kranzer, K., et al., Induction of maturation and cytokine release of human dendritic cells by Helicobacter pylori. Infect Immun, 2004. 72(8): p. 4416-23.

213. Christensen, H.R., H. Frokiaer, and J.J. Pestka, Lactobacilli differentially modulate expression of cytokines and maturation surface markers in murine dendritic cells. J Immunol, 2002. 168(1): p. 171-8.

214. Kimachi, K., M. Croft, and H.M. Grey, The minimal number of antigen-major histocompatibility complex class II complexes required for activation of naive and primed T cells. Eur J Immunol, 1997. 27(12): p. 3310-7.

215. Samoilova, E.B., et al., IL-6-deficient mice are resistant to experimental autoimmune encephalomyelitis: roles of IL-6 in the activation and differentiation of autoreactive T cells. J Immunol, 1998. 161(12): p. 6480-6.

216. Gu, Y., et al., Interleukin 10 suppresses Th17 cytokines secreted by macrophages and T cells. Eur J Immunol, 2008. 38(7): p. 1807-13.

217. Hirata, T., et al., Recruitment of CCR6-expressing Th17 cells by CCL 20 secreted from IL-1 beta-, TNF-alpha-, and IL-17A-stimulated endometriotic stromal cells. Endocrinology, 2010. 151(11): p. 5468-76. 


\section{VITA}

Hossam A. Abdelsamed was born 1979 in Giza, Egypt. He attended and graduated from Ain Shams University in May 2001, with B.Sc. (Honors) in Biochemistry. Following graduation, he worked as a teaching assistant in University of Fayoum, Department of Chemistry, Biochemistry Division to teach Clinical Biochemistry laboratory courses. In August 2005, he entered the Integrated Program of Biomedical Sciences (IPBS) at University of Tennessee Health Science Center. He joined Dr. Kotb's laboratory studying the pathogenesis of Group A Streptococcous (GAS). Following her transition to University of Cincinnati, Hossam preferred to stay in Memphis and joined Dr. Fitzpatrick's laboratory. In her lab, he was studying the T cell response and factors controlling it during Hypersensitivity Pneumonitis. Hossam defended his Ph.D. dissertation successfully in January 2012 then joined Dr. Marion's laboratory and Regional Biocontainment Laboratory (RBL) as a postdoctoral fellow. In his spare time, he enjoys jogging, playing soccer, and reading. 